VERSION: 07 March 2002; Accepted to ApJ

\title{
The Luminosity and Mass Function of the Trapezium Cluster: From B Stars to the Deuterium Burning Limit.
}

\author{
August A. Muench and Elizabeth A. Lada \\ Department of Astronomy, University of Florida, Gainesville, FL 32611 \\ muencheastro.ufl.edu, ladalastro.ufl.edu \\ Charles J. Lada ${ }^{1}$ \\ Harvard-Smithsonian Center for Astrophysics, Cambridge, MA 02138 \\ clada@cfa.harvard.edu \\ and \\ João Alves ${ }^{1}$ \\ European Southern Observatory, Karl-Schwartzschild-Strasse 2, 85748 Garching Germany \\ jalvesdeso.org
}

\begin{abstract}
We use the results of a new, multi-epoch, multi-wavelength, near-infrared census of the Trapezium Cluster in Orion to construct and to analyze the structure of its infrared (K band) luminosity function. Specifically, we employ an improved set of model luminosity functions to derive this cluster's underlying Initial Mass Function (IMF) across the entire range of mass from OB stars to sub-stellar objects down to near the deuterium burning limit. We derive an IMF for the Trapezium Cluster that rises with decreasing mass, having a Salpeter-like IMF slope until near $\sim 0.6 \mathrm{M}_{\odot}$ where the IMF flattens and forms a broad peak extending to the hydrogen burning limit, below which the IMF declines into the sub-stellar regime. Independent of the details, we find that sub-stellar objects account for no more than $\sim 22 \%$ of the total number of likely cluster members. Further, the sub-stellar Trapezium IMF breaks from a steady
\end{abstract}

\footnotetext{
${ }^{1}$ Visiting Astronomer, European Southern Observatory
} 
power-law decline and forms a significant secondary peak at the lowest masses (10-20 times the mass of Jupiter). This secondary peak may contain as many as $\sim 30 \%$ of the sub-stellar objects in the cluster. Below this sub-stellar IMF peak, our KLF modeling requires a subsequent sharp decline toward the planetary mass regime. Lastly, we investigate the robustness of pre-main sequence luminosity evolution as predicted by current evolutionary models, and we discuss possible origins for the IMF of brown dwarfs.

Subject headings: infrared: stars — open clusters and associations: individual (Trapezium Cluster) — stars: luminosity function, mass function — stars: low-mass, brown dwarfs — stars: pre-main sequence

\section{Introduction}

Little is known about the similarities or differences between the star formation process that created the first generation of stars in the universe and the process that is forming stars in nearby stellar nurseries today. One important diagnostic for studying any evolution of the star formation process is the statistical distribution of stellar masses at birth, or the stellar initial mass function (IMF). Comparisons of the mass functions of stars in globular clusters, in the field, in intermediate age open clusters such as the Pleiades and in extremely young clusters embedded in nearby molecular clouds might reveal similarities or differences that would test the notion of a universal mass function (or a dominant star formation process) or that could bring about a better understanding of its stochastic nature (see for example, Kroupa 2001; Gilmore 2001, for recent discussions). Because the initial mass function is an intrinsically statistical quantity, such comparisons require numerous measurements of the IMF in a variety of environments, in turn, requiring tools that can probe the IMF over a large volume of space and time.

Very young embedded clusters are particularly valuable for IMF studies. A simple photomet-

ric census of the members of a young embedded cluster yields a statistically significant population of stars sharing a common heritage (e.g., age and metallicity). Further such a census is relatively complete because young clusters have not lost significant numbers of members to either dynamical or stellar evolution; hence, the observed mass function is the cluster's initial mass function. Because very young clusters are still embedded within their parental molecular cloud, an infrared photometric census is often necessary to identify a complete cluster population. One direct product of such an IR census is the young cluster's stellar infrared luminosity function, which can be used as a tool for studying a cluster's IMF. This may be a particularly effective tool for studying the low mass end of a cluster's IMF because infrared luminosities are relatively easy to derive for brown dwarf cluster members since such intrinsically red sub-stellar sources are at brighter luminosities 
than at any subsequent point in their evolution. Further, modern infrared cameras on modest sized telescopes can efficiently survey numerous young clusters, deriving infrared luminosities for complete populations, and, thus, potentially sampling the IMF of the current epoch over a relatively large volume of the local galaxy.

In our initial work, Muench, Lada \& Lada (2000, hereafter MLL2000), we performed a series of numerical experiments aimed at testing the usefulness of infrared luminosity functions for deriving cluster IMFs. By varying the different fundamental physical properties of a very young cluster, e.g., the cluster's underlying initial mass function (IMF), the cluster's star forming history (SFH) and the appropriate (fixed metallicity) mass-luminosity relation (M-L relation) for cluster members, we showed that a cluster's luminosity function is considerably more sensitive to the form of the underlying initial mass function than to any other parameter. In particular, we found that differences between various theoretical pre-main sequence evolutionary calculations produced only negligible differences in the resulting model luminosity functions and that these differences would not likely be observable. As we show here, this is the result of the fact that canonical PMS luminosity evolution is a relatively robust prediction of current PMS models.

In MLL2000, we applied these results to the infrared (K band) luminosity function (KLF) of the famous Trapezium Cluster in Orion. It is the richest young cluster within the local kiloparsec (Hillenbrand 1997); therefore, it provides a nearby statistically significant sample of sources for which infrared luminosities can be derived, including even the faintest planetary mass members. This is an ideal laboratory for applying and for testing our model luminosity function method. Using the cluster's mean age and age spread taken from spectroscopic observations of optically visible cluster members, we used our method to derive the Trapezium Cluster IMF from its KLF. In MLL2000, we found the cluster's IMF rose steeply in number with decreasing mass until just less than one solar mass where the IMF then flattened and peaked near the hydrogen burning limit before falling throughout the sub-stellar regime with a brown dwarf IMF slope ${ }^{2}$ of $\sim+1$. We also found good agreement between the form of the Trapezium IMF derived from its KLF and that IMF derived by Hillenbrand (1997) for this cluster using a spectroscopic analysis of the optically visible members. Further, luminosity function modeling enabled the derivation of the sub-stellar IMF, which was not possible from the optical/spectroscopic analysis.

In addition to the three fundamental quantities (age, IMF, M-L relation) that govern the structure of a young cluster's luminosity function, there are a number of observational characteristics that may affect the conversion of a cluster's infrared luminosity function into the cluster's IMF.

\footnotetext{
${ }^{2}$ In all cases we will refer to the mass function not the mass spectrum of stars. The mass function is the number of stars per unit volume per unit $\log$ mass The Salpeter (1955) mass function would be a power-law $\xi\left(\log \left(m_{\star}\right)\right)=m^{\Gamma}$ with an index $\Gamma=-1.35$ in this definition.
} 
In particular, very young stars are reddened due to extinction from the parental molecular cloud and from excess infrared emission arising from a circumstellar disk. Cluster membership is often poorly known, requiring a statistical estimate of the interloping field star population. We did not include these observational effects into our MLL2000 derivation of the Trapezium IMF because we had access to only a monochromatic KLF taken from the literature with no color or completeness information. In this current paper, we account for these observational effects by employing a new multi-wavelength near-infrared census of the Trapezium Cluster that we have constructed over a three year period of observations. Using these deeper, more complete observations, we study the contribution of background field stars to the Trapezium Cluster's near-infrared luminosity function, expand our model luminosity function efforts to account for source reddening, and explore the Trapezium KLF at fainter magnitudes than was possible in MLL2000. We re-derive the Trapezium Cluster IMF from its luminosity function, providing a detailed range of possible IMF parameters. We discuss the relative degeneracy of the theoretical mass-luminosity relations derived from different sets of evolutionary models, and we compare the results of several published derivations of the Trapezium IMF that rely upon infrared photometry. Lastly, we discuss possible origins for a derived feature in the Trapezium sub-stellar IMF near the planetary mass regime.

\section{The Trapezium Cluster K band Luminosity Function}

\subsection{The Near-Infrared Census}

To derive a complete multi-wavelength census of the sources in the Trapezium Cluster, we performed infrared observations during 1997 December, 1998 November and 2000 March using two telescopes: the 1.2m telescope at the Fred Lawrence Whipple Observatory (FLWO) at Mt. Hopkins, Arizona (USA) and the ESO 3.5m New Technology Telescope (NTT) in La Silla, Chile. These observations yielded the multi-epoch, multi-wavelength FLWO-NTT infrared catalog that contains $\sim 1000$ sources (see Appendix A for catalog details). Subsets of this catalog have been previously used in the Lada et al., (2000) and Muench et al., (2001) studies of the frequency of circumstellar disks around stars and brown dwarfs in the Trapezium Cluster.

We restrict our subsequent analysis of the cluster's luminosity and mass function to the area surveyed by our deeper NTT observations. We display a $\mathrm{JHK}_{\mathrm{S}}$ color composite image of the $5^{\prime} \times 5^{\prime}$ NTT region in figure 1 . Our observations detected 749 sources within this region. The completeness of this sample at the faintest magnitudes is difficult to quantify because of the spatially variable nebular background. The formal $10 \sigma$ detection limits of our NTT images are 19.75 at $\mathrm{J}, 18.75$ at $\mathrm{H}$ and 18.10 at $\mathrm{K}_{\mathrm{S}}$ based upon the pixel to pixel noise in non-nebulous off-cluster observations that were taken adjacent in time to the on-cluster images. To estimate our actual com- 
pleteness limits, we performed artificial star experiments by constructing a stellar PSF for each of our images and using the IRAF ADDSTAR routine to place synthetic stars in both the off-cluster and the nebulous on-cluster images. A small number of synthetic stars (30-70) with a range of input magnitudes were randomly added across each image and were then recovered using the DAOFIND routine. This was repeated a large number of times (40-200) to achieve sufficient signal to noise for these tests. In off-cluster images, the derived $90 \%$ completeness limits agreed well with the estimated $10 \sigma$ detection limits. In the on-cluster images, the completeness limits were reduced to $90 \%$ completeness limits of $\mathrm{J} \sim 18.15, \mathrm{H} \sim 17.8$, and $\mathrm{K}_{\mathrm{S}} \sim 17.5$ with slightly brighter limits in the dense central core $\left(0.5^{\prime}\right.$ radius from $\theta^{1} \mathrm{C}$ Orionis $)$. We also carefully compared our source list to those published by other recent surveys for the NTT region. To our resolution limit, we detected all the sources found by the Hillenbrand \& Carpenter (2000, hereafter, HC2000) Keck survey except for one, all but two sources from the Luhman et al., (2000) NICMOS survey, but we could not identify nine sources listed in Lucas \& Roche (2000, hereafter, LR2000) UKIRT survey. Further, it is our finding of 58 new sources within our NTT region and unreported by prior catalogs that adds support to the deep and very sensitive nature of our census.

\subsection{Constructing Infrared Luminosity Function(s)}

The FLWO and NTT observations overlap considerably in dynamic range with 504 stars having multi-epoch photometry. For our analysis, we preferentially adopt infrared luminosities from the NTT photometry because it has higher angular resolution and it is an essentially simultaneous set of near-infrared data. For 123 stars that are saturated in one or more bands on the NTT images, the FLWO photometry was used. This transition from NTT to FLWO photometry is at approximately $\mathrm{J}=11.5, \mathrm{H}=11.0$, and $\mathrm{K}=11.0$. For the brightest $5 \mathrm{OB}$ stars, saturated on all our images, we used JHK photometry from the Hillenbrand et al., (1998) catalog. Photometric differences between the FLWO and NTT datasets are small (see Appendix A) and will not affect our construction of the Trapezium Cluster infrared luminosity function(s).

In Figure 2, we present the raw infrared Trapezium Luminosity Functions (LFs). We use relatively wide bins (0.5 magnitudes) that are much larger than the photometric errors (see Appendix A.2). In Figure 2(a), we compare the $J$ and $H$ band LFs for stars in this region. In the Figure 2(b), we compare the K band LF of the NTT region to that K band LF constructed in MLL2000. As was observed in previous studies of the Trapezium Cluster, the cluster's infrared luminosity function (J, $\mathrm{H}$, or $\mathrm{K}$ ) rises steeply toward fainter magnitudes, before flattening and forming a broad peak. The

LF steadily declines in number below this peak but then rapidly tails off a full magnitude above our completeness limits.

For our current derivation of the Trapezium IMF, we use the Trapezium K band Luminosity 
Function, rather than the $\mathrm{J}$ or H LFs. We do so in order to minimize the effects of extinction on the luminosities of cluster members (see section 3.1.1), to maximize our sensitivity to intrinsically red, low luminosity brown dwarf members of this cluster, and to make detailed comparisons to our work in MLL2000. For example, the new FLWO-NTT Trapezium KLF contains significantly more stars at faint $(K>14)$ magnitudes than the KLF used in MLL2000. Interestingly, a secondary peak near K = 15 seen in the MLL2000 KLF (originally McCaughrean et al., 1995) is much more significant and peaks at $\mathrm{K}=15.5$ in the new FLWO-NTT KLF. Similar peaks are not apparent in the J or H band LFs constructed here, though Lucas \& Roche (2000) report a strong secondary peak in their Trapezium JLF. Such secondary peaks in young cluster luminosity functions have often been evidence of a background field star population contributing to the source counts (e.g., Luhman et al., 1998; Luhman \& Rieke 1999).

To account for the possible field star contamination, we systematically obtained images of control fields away from the cluster and off of the Orion Molecular Cloud. The FLWO offcluster field(s) were centered at approximately R.A. $=05^{h} 26^{m}$; DEC. $=-06^{\circ} 00^{\prime}(\mathrm{J} 2000)$ and were roughly twice the area of the NTT off-fields. The NTT off-cluster region was centered at R.A. = $05^{h} 37^{m} 43$ s.7; DEC. $=-01^{\circ} 55^{\prime} 42^{\prime \prime} 7$ (J2000). Figure 3(a) displays the two off-field KLFs (scaled to the same area) from these observations and in the inset, their $(\mathrm{H}-\mathrm{K})$ distributions. The relatively narrow $(\mathrm{H}-\mathrm{K})$ distributions indicate that the two off-fields sample similar populations and that they are un-reddened. We constructed an observed field star KLF by averaging these luminosity functions, weighting (by area) toward the FLWO off-fields for K brighter than 16th magnitude, the completeness limit of that dataset, and toward the more sensitive NTT off-fields for fainter than K $=16$. In Figure 3(b), we compare the resulting field star KLF to the Trapezium KLF of the NTT region. It is plainly apparent from the raw control field observations that while field stars may contribute to the Trapezium Cluster IR luminosity function over a range of magnitudes, their numbers peak at magnitudes fainter than the secondary peak of the on-cluster KLF and do not appear sufficient in number to explain it.

\subsection{Defining a Complete Cluster KLF}

We determine the completeness of our FLWO-NTT Trapezium Cluster KLF by constructing and by analyzing the cluster's infrared ( $\mathrm{H}$ - K) versus K color-magnitude diagram. For the purposes of our analysis, we adopt the same cluster parameters as we used in MLL2000, e.g., a cluster mean age of 0.8 Myrs (Hillenbrand 1997) and a cluster distance of $400 \mathrm{pc}$. As seen in figure 4(a), the luminosities of the Trapezium sources form a continuously populated sequence from the bright $\mathrm{OB}$ members $(\mathrm{K} \sim 5)$ through sources detected below our completeness limits.

To interpret this diagram, we compare the locations of the FLWO-NTT sources in color- 
magnitude space to the cluster's mean age isochrone as derived from theoretical pre-main sequence (PMS) calculations. Because the D'Antona \& Mazzitelli (1997, hereafter, DM97) models include masses and ages representative of the Trapezium Cluster and inorder to provide continuity with our work in MLL2000, we will use these tracks to define a complete cluster sample from figure 4(a). Differences among pre-main sequence tracks should not have significant effect upon our analysis of the color-magnitude diagram (see section 4.3). It is clear from this diagram that the cluster sources are reddened away from the theoretical $0.8 \mathrm{Myr}$ isochrone, which forms a satisfactory left hand boundary to the sources in this color-magnitude space. This isochrone, however, does not span the full luminosity range of the observations and a number $(\sim 40)$ sources lie below the faint end of the DM97 isochrone. As a result, our subsequent analysis that makes use of the DM97 models will be restricted to considering only those sources whose luminosities, after correction for extinction, would correspond to masses greater than the mass limit of the DM97 tracks, i.e., $0.017 \mathrm{M}_{\odot}$ or roughly 17 times the mass of Jupiter $\left(\mathrm{M}_{\text {Jup }}\right)$. Despite the lower mass limit imposed by these PMS tracks, our infrared census spans nearly three orders of magnitude in mass, illustrating the utility of studying the mass function of such rich young clusters.

Extinction acts to redden and to dim sources of a given mass to a brightness below our detection limits. To determine our ability to detect extincted stars as a function of mass, we draw a reddening vector from the luminosity (and color) of a particular mass star on the mean age isochrone until it intersects the $10 \sigma$ sensitivity limit of our census. We can detect the 1 Myr old Sun seen through $\mathrm{A}_{V} \sim 60$ magnitudes of extinction or a PMS star at the hydrogen burning limit seen through $\sim 35$ magnitudes. For very young brown dwarfs at our lower mass limit $\left(17 \mathrm{M}_{\text {Jup }}\right)$, we probe the cloud to $\mathrm{A}_{V}=17$ magnitudes. We use this latter reddening vector as a boundary to which we are complete in mass, and we draw a mass and extinction $\left(M-A_{V}\right)$ limited subset of sources bounded by the mean age isochrone and the $A_{V}=17$ reddening vector and mark these as filled circles in figure $4(\mathrm{a})$. Our $\mathrm{M}-\mathrm{A}_{V}$ limits probe the vast majority of the cluster population, including $81 \%$ of the sources the color-magnitude diagram.

In figure 4(b) we present the $\mathrm{M}-\mathrm{A}_{V}$ limited KLF, containing 583 sources. Thirty-two sources, detected only at $\mathrm{K}$ band (representing only $4 \%$ of our catalog), were also excluded from our further analysis. The median $\mathrm{K}$ magnitude of these sources is $\mathrm{K}=15$, and we expect that these are likely heavily reddened objects. We compare the $\mathrm{M}-\mathrm{A}_{V}$ limited KLF to the unfiltered Trapezium KLF. Clearly, heavily reddened sources contributed to the cluster KLF at all magnitudes and their removal results in a narrower cluster KLF. However, the structure (e.g., peak, slope, inflections, etc.) of the KLF remains largely unchanged. The secondary peak of the cluster KLF between $\mathrm{K} \sim 14-17$ seems to be real since it is present in both the raw and the $\mathrm{M}-\mathrm{A}_{V}$ limited KLFs, though we have not yet corrected for background field stars.

There are at least three possible sources of incompleteness in our mass/extinction limited 
sample. The first arises because sources that are formally within our mass and extinction limits may be additionally reddened by infrared excess from circumstellar disks and, hence, be left out of our analysis. However, this bias will affect sources of all masses equally because infrared excess appears to be a property of the young Trapezium sources over the entire luminosity range (Muench et al., 2001). Second, the Trapezium Cluster is not fully coeval and our use of the cluster's mean age to draw the $\mathrm{M}-\mathrm{A}_{V}$ sample means that cluster members at our lower mass limit $\left(17 \mathrm{M}_{\text {Jup }}\right)$ but older than the cluster's mean age $(\tau>0.8 \mathrm{Myrs})$ will be fainter than the lower boundary and left out of our sample. Further, sources younger than the mean age but below $17 \mathrm{M}_{\text {Jup }}$ will be included into the sample. This "age bias" will affect the lowest mass sources, i.e., $<20 \mathrm{M}_{J u p}$. Third, because of the strong nebular background, our true completeness limit (see section 2.1) is brighter than our formal $10 \sigma$ sensitivity for approximately $60 \%$ of the area surveyed. The resulting sample incompleteness only affects our sensitivity to sources less than $30 \mathrm{M}_{\text {Jup }}$ and with $\mathrm{A}_{V}>10$. We do not correct the Trapezium KLF to account for these effects or biases.

\subsection{Field Star Contamination to the KLF}

The lack of specific membership criteria for the embedded sources in the Trapezium Cluster requires an estimate of the number of interloping non-cluster field stars in our sample. Some published studies, for example LR2000 and Luhman et al., (2000), assume that the parental molecular cloud acts as a shield to background field stars; whereas HC2000 suggests that the background contribution is non-negligible. HC2000 estimates the field star contribution using an empirical model of the infrared field star population and convolving this model with a local extinction map derived from a molecular line map of the region. This approach may suffer from its dependence upon a field star model that is not calibrated to these faint magnitudes and that does not include very low mass field stars. As we show, there are also considerable uncertainties in the conversion of a molecular line map to an extinction map. For our current study, we use our observed K band field star luminosity function (see figure 3) to test these prior methodologies and to correct for the field star contamination. We point out that no such estimate can account for contamination due to young, low mass members of the foreground Orion OB1 association.

We compare in figure 5 the effects of six different extinction models upon our observed field star KLF. In panels A and B, we tested simple Gaussian distributions of extinction centered respectively at $\mathrm{A}_{V}=10$ and 25 magnitudes with $\sigma=5$ magnitudes. In both cases, the reddened field star KLF contains significant counts above our completeness limit and "background extinction shields" such as these do not prevent the infiltration of field stars into our counts. In the second pair of reddened off-fields (panels C and D), we followed the HC2000 prescription for estimating background field stars by convolving our observed field star KLF with the $\mathrm{C}^{18} \mathrm{O}$ map from Goldsmith, Bergin 
\& Lis (1997) converted from column density to dust extinction. We note that there is substantial uncertainty in the conversion from $\mathrm{C}^{18} \mathrm{O}$ column density to dust extinction. There is at least a factor of 2 variation in this conversion value in the literature, where Frerking, Langer \& Wilson (1982) derived a range from $0.7-2.4$ (in units of $10^{14} \mathrm{~cm}^{2} \mathrm{mag}^{-1}$ ) and Goldsmith, Bergin \& Lis (1997) estimated a range of values from $1.7-3$. Either the result of measurement uncertainty or the product of different environmental conditions, this variation produces a factor of 2 uncertainty in the extinction estimates from the $\mathrm{C}^{18} \mathrm{O}$ map. In short, we find that a $\mathrm{C}^{18} \mathrm{O} \rightarrow \mathrm{A}_{V}$ ratio of 3.0 (panel C) results in twice as many interloping background field stars as would a value of 1.7 (panel D; equivalent to that used by HC2000).

In panels $\mathrm{E}$ and $\mathrm{F}$ of figure 5 we derive the same reddened off-field KLFs as in the prior pair, but they have been filtered to estimate the actual contribution of field-stars to our $\mathrm{M}-\mathrm{A}_{V}$ limited sample. These filters, which were based upon on the K brightness of the lower mass limit of our PMS models and on the derived extinction limit of the $\mathrm{M}-\mathrm{A}_{V}$ sample, were applied during the convolution of the field star KLF with the cloud extinction model such that only reddened field stars that would have $\mathrm{A}_{V}<20$ and unreddened $\mathrm{K}$ magnitudes $<16$ would be counted into filtered reddened off-field KLF. The extinction limit was expanded from 17 to 20 magnitudes to account for the dispersion of the H-K distribution of un-reddened field-stars $(\sim 0.2)$. A factor of 2 uncertainty remains. Alves, Lada \& Lada (1999) derive a more consistent estimate of the $\mathrm{C}^{18} \mathrm{O} \rightarrow \mathrm{A}_{V}$ ratio from near-infrared extinction mapping of dark clouds, suggesting a median ratio of 2.1. Adopting $\mathrm{C}^{18} \mathrm{O} \rightarrow \mathrm{A}_{V}=2.1$, we estimate there are $\sim 20 \pm 10$ field stars in our $\mathrm{M}-\mathrm{A}_{V}$ limited KLF. From these experiments, we find, however, that both the raw and reddened off-field KLFs always peak at fainter magnitudes than the secondary peak of the Trapezium KLF, and that the subtraction of these field-star corrections from the Trapezium KLF do not remove this secondary peak. These findings suggest that the secondary KLF peak is a real feature in the Trapezium Cluster's infrared luminosity function.

\section{The Trapezium Cluster Initial Mass Function}

We analyze the Trapezium Cluster's K band luminosity function constructed in section 2 using our model luminosity function algorithm first described in MLL2000. Following our work in MLL2000, our goal is to derive the underlying mass function or set of mass functions whose model luminosity functions best fit the Trapezium Cluster KLF. We have improved our modeling algorithm by including statistical distributions of the reddening properties of the cluster. We have also improved our analysis by applying the background field star correction from section 2.4 and by employing improved fitting techniques for deriving IMF parameters and confidence intervals. Before deriving the cluster IMF, we use the extensive color information available from the FLWO- 
NTT catalog to explore the reddening (extinction and infrared excess) properties of the Trapezium sources. In section 3.1, we use this information to create recipes for deriving the probability distributions functions of extinction and excess which can be folded back into our modeling algorithm during our derivation of the Trapezium IMF. We present the new model luminosity functions and fitting techniques in section 3.2 and summarize the derived IMF in section 3.3.

\subsection{Deriving Distributions of Reddening}

\subsubsection{Extinction Probability Distribution Function}

We use the extensive color information provided by our FLWO-NTT catalog to construct a probability distribution function of the intra-cluster extinctions (hereafter referred to as the Extinction Probability Distribution Function or EPDF) based upon the color excesses of individual Trapezium sources. Because the stellar photospheric $(\mathrm{H}-\mathrm{K})$ color has a very narrow distribution of intrinsic photospheric values it should be the ideal color from which to derive line of sight extinction estimates, as shown, for example, in the Alves et al., (1998) study of the structure of molecular clouds. In figure 6(a) we show the histogram of observed (H - K) color for all our Trapezium Cluster sources. This histogram peaks at $(\mathrm{H}-\mathrm{K})=0.5$ and is quite broad especially when compared to the narrow unreddened photospheric (field-star) $(\mathrm{H}-\mathrm{K}$ ) distributions seen in figure 3(a). This broad distribution may be in part the result of extinction; however, as recently shown in Lada et al., (2000) and Muench et al., (2001), approximately 50\% of the these Trapezium Cluster sources, independent of luminosity, display infrared excess indicative of emission from circumstellar disks. This is illustrated in figure 6(b) where it is clear that there are both heavily reddened sources $\left(\mathrm{A}_{V} \sim 35\right)$ and sources with large infrared excesses (falling to the right of the reddening band for main sequence objects). If the ( $\mathrm{H}-\mathrm{K})$ color excess were assumed to be produced by extinction alone without accounting for disk emission, the resulting extinction estimates would be too large.

Meyer, Calvet \& Hillenbrand (1997) showed that the intrinsic infrared colors of star with disks are confined to a locus (the classical T-Tauri star locus or CTTS locus) in the $(\mathrm{H}-\mathrm{K}) /(\mathrm{J}-\mathrm{H})$ color-color diagram. We derive individual $\mathrm{A}_{V}$ estimates for sources in the $(\mathrm{H}-\mathrm{K}) /(\mathrm{J}-\mathrm{H})$ colorcolor diagram by dereddening these stars back to this CTTS locus along a reddening vector defined by the Cohen et al., (1981) reddening law. Sources without J comprise $\sim 20 \%$ of the catalog and as shown in figure 6 , their $(\mathrm{H}-\mathrm{K})$ colors appear to sample a more heavily embedded population, implying extinctions as high as $\mathrm{A}_{V} \sim 60$. $\mathrm{A}_{V}$ estimates are derived for these sources by assigning a typical star-disk $(\mathrm{H}-\mathrm{K})$ color $=0.5$ magnitudes, and de-reddening that source. Sources near to but below the CTTS locus are assigned an $\mathrm{A}_{V}=0$. The individual extinctions are binned into an extinction probability distribution function (EPDF) as shown in figure 7(a). Also shown are 
the effects of changing the typical star-disk $(\mathrm{H}-\mathrm{K})$ color assumed for those stars without $\mathrm{J}$ band. Little change is seen. Compared to the cloud extinction distribution function, which was integrated

over area from the $\mathrm{C}^{18} \mathrm{O}$ map, the cluster EPDF is very non-gaussian and peaks at relatively low extinctions, $\mathrm{A}_{V}=2.5$, having a median $\mathrm{A}_{V}=4.75$ and a mean $\mathrm{A}_{V}=9.2$. Further, the cluster EPDF is not well separated from the reddening distribution provided by the molecular cloud. Rather the cluster population significantly extends to extinctions as high as $\mathrm{A}_{V}=10-25$, near and beyond the peak of the cloud extinction function. Ancillary evidence of this significant population of heavily reddened stars is seen in the color-color diagram (figure 6b) which clearly illustrates the extension of the cluster to regions of the molecular cloud with $\mathrm{A}_{V}>10$. Lastly, it is clear that the deep nature of our survey has allowed us to sample both the majority of the embedded cluster, and the cloud over the full range of density.

In our revised model luminosity function algorithm, we randomly sample the cluster's EPDF to assign an $\mathrm{A}_{V}$ to each artificial star in the model LF. The effect of the EPDF on the model luminosity function is wavelength and reddening law (Cohen et al., 1981, in this case) dependent. In figure 8 we construct model I, J, H, and K luminosity functions, reddening each by the Trapezium Cluster EPDF. The effect of the EPDF on the intrinsic I and J band LFs is profound, rendering the reddened I band LF almost unrecognizable. Yet at longer wavelengths, specifically at $\mathrm{K}$ band, the effects of extinction are minimized. We note that the overall form of the reddened model $\mathrm{K}$ band luminosity function has not been changed by the Trapezium EPDF in a significant way, e.g., the peak of the model KLF is not significantly blurred and the faint slope of the KLF has not been changed from falling to flat. This suggests that our modeling of the Trapezium KLF in MLL2000, which did not account for reddening due to extinction, is generally correct. However, we likely derived too low of a turnover mass for the Trapezium IMF because reddening shifted the intrinsic LF to fainter magnitudes.

\subsubsection{Infrared Excess Probability Distribution Function}

Because we wish to use the Trapezium K band LF to minimize the effects of extinction, we must also account for the effects of circumstellar disk emission at $\mathrm{K}$ band. The frequency distribution of the resulting excess infrared flux is not a well known quantity, and when previously derived, it has depended significantly upon additional information derived from the spectral classification of cluster members (Hillenbrand \& Carpenter 2000; Hillenbrand et al., 1998). One of the goals of this present work is to construct a recipe for deriving the $\mathrm{K}$ band excess distribution directly from the infrared colors of the cluster members.

To derive a first-order infrared excess probability distribution function (IXPDF) for the Trapezium Cluster sources, we simply assume that any excess $(\mathrm{H}-\mathrm{K})$ color (above the photosphere, after 
removing the effects of extinction) reflects an excess at $K$ band alone, realizing this may underestimate the infrared excess of individual sources. We only use the sources having JHK measurements and lying above the CTTS locus in the color-color diagram. We remove the effects of extinction from each source's observed $(\mathrm{H}-\mathrm{K})$ color using the same method described above, i.e., dereddening back to the CTTS locus. However, the photospheric $(\mathrm{H}-\mathrm{K})$ color for each star cannot be discreetly removed from this data alone. The photospheric infrared colors of pre-main sequence stars appear to be mostly dwarf-like (Luhman 1999), and therefore, we used the observed field star $(\mathrm{H}-\mathrm{K})$ distribution shown in figure 3(a) as a probability distribution of photospheric values. We derive the IXPDF by binning the de-reddened $(\mathrm{H}-\mathrm{K})$ colors into a probability function and then subtracting the distribution of photospheric colors using a Monte Carlo integration.

The Trapezium Cluster IXPDF is shown in figure 7(b). The IXPDF peaks near 0.2 magnitudes with a mean $=0.37$, a median $=0.31$, and a maximum excess of $\sim 2.0$ magnitudes. Probabilities of negative excesses were ignored. The IXPDF is similar to the $(\mathrm{H}-\mathrm{K})$ excess distribution shown in HC2000 and derived in Hillenbrand et al., (1998) yet extends to somewhat larger excess values. Each artificial star in our models is randomly assigned a $\mathrm{K}$ band excess (in magnitudes) drawn from the IXPDF.

\subsection{Modeling the Trapezium Cluster KLF}

To model the Trapezium Cluster KLF, we apply the appropriate field star correction derived in section 2.4 to the $\mathrm{M}-\mathrm{A}_{V}$ limited KLF constructed in section 2.3. We fix the Trapezium Cluster's star forming history and distance to be identical to that used in MLL2000. Specifically, these are a distance of 400pc (m-M=8.0) and a star forming history characterized by constant star formation from 1.4 to 0.2 Myrs ago, yielding a cluster mean age of 0.8 Myrs (Hillenbrand 1997) and an age spread of 1.2 Myrs. We adopt our standard set of merged theoretical pre-main sequence tracks from MLL2000 ${ }^{3}$. Our merged standard set of tracks span a mass range from 60 to $0.017 \mathrm{M}_{\odot}$, allowing us to construct a continuous IMF within this range. We incorporated the cluster's reddening distributions derived in section 3.1 into our modeling algorithm and chose a functional form of the cluster IMF similar to that used in MLL2000; specifically, an IMF constructed of powerlaw segments, $\Gamma_{i}$ connected at break masses, $m_{j}$. As in MLL2000, we found that an underlying 3 power-law IMF produced model KLFs that fit the observations over most of the luminosity range,

\footnotetext{
${ }^{3}$ Our standard set of theoretical tracks are described in Appendix B of MLL2000: they are a merger of evolutionary calculations including a theoretical Zero Age Main Sequence (ZAMS) from Schaller et al., (1992), a set of intermediate mass (1-5 $\mathrm{M}_{\odot}$ ) "accretion-scenario" PMS tracks from Bernasconi (1996), and the low mass standard deuterium abundance PMS models from D’Antona \& Mazzitelli (1997) for masses from 1 to $0.017 \mathrm{M}_{\odot}$
} 
corresponding to masses from 5 to $0.03 \mathrm{M}_{\odot}$. In section 3.2.1, we utilize our $\chi^{2}$ minimization routine to identify those 3 power-law IMFs that best fit the observed KLF within this mass range, and we estimate confidence intervals for these IMF parameters in section 3.2.2. We find that the faint Trapezium brown dwarf KLF, corresponding to masses less than $0.03 \mathrm{M}_{\odot}$, contains structure and a secondary peak that are not well fit by the 3 power-law IMF models. In section 3.2.3 we model this secondary KLF peak using a corresponding break and secondary feature in the cluster brown dwarf IMF between 0.03 and $0.01 \mathrm{M}_{\odot}$.

\subsubsection{Results of $\chi^{2}$ Fitting: Best Fit Three Power-Law IMFs}

Our $\chi^{2}$ minimization procedure calculates the reduced $\chi^{2}$ statistic and probability for a particular model KLF fit to the Trapezium KLF over a range of magnitude bins. Parameters for the underlying three power-law IMF are taken from the best fit model KLFs, and we fit both reddened and unreddened model KLFs. The 3 power-law IMF derived from these fits is summarized in table 1. We found that the results of our model fits were dependent upon the dynamic range of K magnitude bins over which the models were minimized. Specifically, we find that our results are very sensitive to the formation of a secondary peak in the Trapezium KLF at $K=15.5$, which remains despite the subtraction of the field star KLF.

We derive good model KLF fits $\left(\chi^{2}\right.$ prob $\left.\sim 1\right)$ when fitting between the $K=7.5$ bin and the $\mathrm{K}=14.5$ bin (see figure 9a), the same luminosity range we modeled in MLL2000. Within the this fit range we find an optimal Trapezium IMF nearly identical to that found in MLL2000 even after accounting for reddening. The derived IMF rises steeply from the most massive stars with $\Gamma_{1}=-1.3$ before breaking to a shallower IMF slope of $\Gamma_{2}=-0.2$ at $0.6 \mathrm{M}_{\odot}\left(\log m_{1} \sim-0.2\right)$. The derived IMF peaks near the hydrogen burning limit $\left(0.10-0.08 \mathrm{M}_{\odot}\right.$ or $\left.\log m_{2}=-1.0-1.1\right)$ and then breaks and falls steeply throughout the brown dwarf regime with $\Gamma_{3} \sim+1.0$. We also derive good fits to $K=15$ (just before the secondary peak in the cluster KLF), with the resulting IMF peaking at slightly higher masses $\left(0.13-0.10 \mathrm{M}_{\odot}\right)$ and falling with a slightly shallower slope, $\Gamma_{3} \sim+0.7$ to 0.8 . The unreddened luminosities of this fit range correspond to a mass range from 5.0 to $0.03 \mathrm{M}_{\odot}$.

However, we cannot produce models KLFs based upon a three power-law IMF that adequately fit the secondary peak in the Trapezium KLF. For example, our best fit to the secondary peak in figure 9(b) is inconsistent with the overall form of the faint KLF, being unable to replicate both the falling KLF at $K=14.5$ nor the secondary peak at $K=15.5$. Such structure in the faint Trapezium KLF implies similar non-power law structure in the underlying IMF, while our current models based upon a three power-law IMF essentially assign a single power-law IMF slope for the entire brown dwarf regime. We will explore this structure in the faint brown dwarf KLF and IMF in 
section 3.2.3, but first we examine the confidence intervals for the derived 3 power-law IMFs.

\subsubsection{Results of $\chi^{2}$ Fitting: Range of Permitted Three Power-Law IMFs}

Our $\chi^{2}$ fitting routine also allows us to investigate the range of permitted cluster IMFs from modeling the cluster KLF. We illustrate the range of IMFs and the effects of source reddening on our fits in figure 10 and summarize the corresponding constraints on the IMF parameters in table 1. In each panel, we plot the contours of $\chi^{2}$ probability for two of the 5 dependent IMF parameters while restricting the other three parameters to a best fit model. In each panel we also display contours for fits with (solid) and without (dashed) source reddening, and we examine the dependence of these parameters for models fit to the $K=14.5$ and $K=15.0$ bins.

In all our fitting experiments (here and MLL2000), the high mass slope of the cluster IMF, $\Gamma_{1}$, was well constrained with slopes measured between -1.0 and -1.3. Based on this result, we fix $\Gamma_{1}$ to equal -1.3. Panels (a) - (c) in figure 10 display the ranges of the other 4 IMF parameters when fitting to a K limit $=14.5$. Panel (a) plots the dependence of the two break masses, $m_{1}$ and $m_{2}$. The fits for these parameters are well behaved with $90 \%$ contours have a typical width of 0.1 0.2 dex in units of log mass. Source reddening has two clear effects upon our fit results. When source reddening is included, the high mass break, $m_{1}$, decreases and the low mass break, $m_{2}$, increases. The second effect is that the size of the $90 \%$ confidence contour increases when source reddening is included into the model fits. Panel (b) displays the dependence of the low mass break, $m_{2}$, on the middle power-law slope, $\Gamma_{2} . \Gamma_{2}$ is fairly well constrained to be slightly rising to lower masses, and the permitted range of $m_{2}$ is again roughly $0.1-0.2$ dex, centered near 0.1 $\mathrm{M}_{\odot}(\log m \sim-1)$. Accounting for source reddening again shifts the low-mass break to slightly higher masses, increases the size of the $90 \%$ contour, and in this case, flattens the central powerlaw.

Panel (c) displays the dependence of $\Gamma_{3}$ upon the second break mass, $m_{2}$. Though $m_{2}$ is fairly well constrained to have values between 0.13 and $0.08 \mathrm{M}_{\odot}$, the low mass power-law slope, $\Gamma_{3}$, has a large range of possible slopes from 0.50 to 1.50 within the $90 \% \chi^{2}$ contour for models with source reddening. Panel (d) plots the same parameters as panel (c) but for fits to the $\mathrm{K}$ limit $=15$. These fits give somewhat flatter $\Gamma_{3}$ slopes and somewhat higher mass $m_{2}$ breaks, but are actually slightly better constrained. As discussed in the previous section, our model KLFs employing a 3

power-law IMF do not provide good fits to the secondary peak in the KLF. As the fit range shifts to fainter magnitudes, $\Gamma_{3}$ flattens, but the total $\chi^{2}$ confidence depreciates due to the secondary peak. We explore the IMF parameters necessary to fit this secondary peak in the next section. 


\subsubsection{Fitting the Secondary Peak in the Trapezium Cluster KLF}

In contrast to our expectations in MLL2000, the departure from a power-law decline and the formation of a secondary peak at the faint end of the Trapezium KLF remains after correcting for reddened background field stars. When we attempt to fit the faint KLF using an underlying three power-law IMF, we find that our model KLFs, while producing excellent fits over the majority of the Trapezium KLF, could not simultaneously reproduce the formation of the secondary peak. Since there is no known corresponding feature in the mass-luminosity relation (see section 4.2), we hypothesize that the KLF's break from a single continuous declining slope at $\mathrm{K}>14.5$ (M < $30 \mathrm{M}_{\text {Jup }}$ ) and the formation of a secondary KLF peak directly imply a similar break and feature in the cluster IMF. Further, the rapid tailing off of the cluster KLF below this secondary peak also directly implies a similar rapid decline or truncation in the underlying IMF, as was also discussed in LR2000.

We modeled the secondary KLF peak by adding a fourth, truncated, power-law segment, $\Gamma_{4}$, to the three power-law IMFs derived in section 3.2.1. The truncation of the fourth power-law segment enabled us to model the rapid tailing off of the cluster KLF below the secondary peak, but was also dictated by the artificial low mass cut off present in the adopted merged PMS tracks, which for the sub-stellar regime come from DM97. As such, the truncation mass of the model IMF was arbitrarily set to $0.017 \mathrm{M}_{\odot}$. We found that this 4 power-law truncated IMF produced good $\chi^{2}$ model KLF fits to the secondary KLF peak. The best fit model KLF shown in figure 11(a) has an underlying brown dwarf IMF that breaks from a steady decline at $m_{3}=0.025 \mathrm{M}_{\odot}$ and then rises steeply with $\Gamma_{4} \sim-5$ before truncating at the lower mass limit. Examination of the confidence intervals for the $m_{3}$ and $\Gamma_{4}$ parameters showed that higher mass breaks $\left(m_{3} \sim 0.035\right)$ required flatter $\Gamma_{4}$ slopes, but they had worse $\chi^{2}$ and peaked before the observed peak at $\mathrm{K}=15.5$. This suggests that were the slope of the mass-luminosity relation continuous (and constant) toward lower masses, the exact location of the secondary IMF peak would shift to somewhat lower masses than we can derive using the truncated PMS tracks.

\subsection{Derived Trapezium Cluster IMF}

\subsubsection{The Overall Cluster IMF}

Figure 11(b) shows our overall best fit Trapezium IMF and graphically displays the range of cluster IMFs permitted by our modeling of the Trapezium KLF using our standard set of merged PMS tracks. We adopt the following four power-law function with a truncation at the lowest masses for the underlying IMF of the Trapezium: 


$$
\frac{d N}{d \log m}=\left\{\begin{array}{lll}
M_{\star}^{\Gamma} & ; \quad \Gamma=\left\{\begin{array}{lll}
-1.21 & : & M_{\star}>0.600 \mathrm{M}_{\odot} \\
-0.15 & : & 0.600 \mathrm{M}_{\odot}>M_{\star}>0.120 \mathrm{M}_{\odot} \\
+0.73 & : & 0.120 \mathrm{M}_{\odot}>M_{\star}>0.025 \mathrm{M}_{\odot} \\
-5.00 & : & 0.025 \mathrm{M}_{\odot}>M_{\star}>0.017 \mathrm{M}_{\odot}
\end{array}\right. \\
0 \quad ; \quad M_{\star}=0.017 \mathrm{M}_{\odot}
\end{array}\right.
$$

We find that despite the use of deeper, more complete observations, the application of detailed mass and extinction limits to the cluster sample, the inclusion of source reddening into the model luminosity function algorithm and the correction of our infrared census for reddened field stars, our derived Trapezium IMF is not a significant revision over that found in MLL2000. We find that the inclusion of source reddening into our modeling algorithm, while providing a more accurate representation of the cluster properties, results in cluster IMFs that have very similar power-law slopes and break masses as fits without source reddening, especially when fit to the same luminosity range. Source reddening does indeed blur the precision of the IMFs we can derive. For example, our MLL2000 IMF derived without accounting for source reddening is somewhat broader and peaks to slightly lower mass than the IMF derived here with source reddening. Though the overall derived IMF has not significantly changed from our findings in MLL2000, our more complete infrared census and improved understanding of the field-star population do allow us to explore the Trapezium IMF at lower masses than MLL2000. We find that the secondary peak of the observed Trapezium KLF is not the result of background field stars, and we derive a corresponding secondary peak in the low mass brown dwarf IMF between 10 and $30 \mathrm{M}_{\text {Jup }}$. However, because of the restriction imposed by the low mass limit of the PMS tracks, both the precise location and amplitude of the secondary peak and the precise form of the IMF below $17 \mathrm{M}_{J u p}$ are somewhat uncertain.

\subsubsection{The Sub-Stellar IMF, A Closer Look}

To better define the secondary peak in the Trapezium IMF, we consider only the sub-stellar regime of the Trapezium KLF ( $\mathrm{K} \geq 13$ ), where we can employ a different set of PMS tracks that cover the corresponding brown dwarf regime but also extend to masses less than the limit of our standard merged PMS tracks. The Burrows et al., (1997, hereafter, B97) PMS models are available from 0.12 to $0.001 \mathrm{M}_{\odot}\left(1 \mathrm{M}_{\text {Jup }}\right)$, and for the relevant age range of the Trapezium. While the mass to K luminosity relation is relatively robust between different sets of PMS tracks, the B97 PMS models do display a somewhat flatter mass-luminosity relation for sub-stellar objects than do DM97. We rederived the cluster's $\mathrm{M}-\mathrm{A}_{V}$ limited, background corrected Trapezium KLF following our prescription in section 2.3 but using the B97 isochrone. Since the nebular background decreases 
our survey's completeness to heavily reddened $\left(\mathrm{A}_{V}>10\right)$, very low mass $\left(\mathrm{M}<0.03 \mathrm{M}_{\odot}\right)$ brown dwarfs, we draw this $\mathrm{M}-\mathrm{A}_{V}$ sample to our completeness limit rather than our $10 \sigma$ sensitivity limit to ensure the precision of the sub-stellar IMF. The resulting $\mathrm{M}-\mathrm{A}_{V}$ limited KLF from the extended B97 isochrone samples the cluster population to a predicted mass limit of $0.01 \mathrm{M}_{\odot}$ and an $\mathrm{A}_{V}=9$.

New model KLF fits (see figure 12a) that employ the B97 tracks and use a three power-law underlying sub-stellar IMF yield a power-law brown dwarf IMF falling with a similar but somewhat steeper slope than our standard tracks (see summarized IMF parameters in table 2) ${ }^{4}$. Similar to DM97, the B97 tracks require the presence of a significant secondary peak that departs from the power-law function at $m_{3} \sim 0.02 \mathrm{M}_{\odot}$ and rises very steeply with $\Gamma_{4}=-5$ as shown in figure 12b. Further, the extended mass range of the B97 tracks allows us to resolve the location of the secondary peak: our fits require a peak near the deuterium burning limit, i.e., $13-14 \mathrm{M}_{\text {Jup }}$, followed by a rapidly declining IMF with a slope of $\Gamma_{5}=+5$ down to $10 \mathrm{M}_{\text {Jup }}$. The sharp decline in the substellar IMF below this peak is not the result of the application of the off-field correction. Fits to Trapezium sub-stellar KLF without correcting for background field stars yeild nearly identical cluster IMFs (see figure 12a and table 2). Further, the sharp decline in our derived sub-stellar IMF below the deuterium burning limit and independent of the background correction confirms a similar, straightforward interpretation of the observed rapid turn down in the cluster KLF and dearth of sources in the $(\mathrm{H}-\mathrm{K}) / \mathrm{K}$ color magnitude diagram nearly a full magnitude above our completeness limits (see figure 4 ).

Lastly, this feature in the IMF appears to be a statistically significant departure from the power-law decline of the brown dwarf IMF as was implied by our $\chi^{2}$ fitting in section 3.2.1. We ran a Monte Carlo simulation of the derived Trapezium sub-stellar IMF for a population of 150 brown dwarfs. In figure 13 we show the resulting histogram form of the average simulated cluster IMF. Using equally sized bins in log mass units, we calculated the statistical variation in an IMF bin as a function of 50 drawn samples. From these plotted $1 \sigma$ error bars, it is clear that the derived secondary peak is a significant statistical result. In addition, these results imply that a statistically significant identification of such a feature at the tail of the IMF requires the examination of a rich sub-stellar population such as that provided by the Trapezium Cluster.

\footnotetext{
${ }^{4}$ Fiting the sub-stellar sample drawn to an $\mathrm{A}_{V}<17$ with model LFs using B97 tracks yielded a power-law decline closer to that derived from our standard merged models
} 


\section{Discussion}

\subsection{The Structure of the Trapezium KLF and IMF}

\subsubsection{The Stellar Regime}

From our careful construction and improved modeling of the Trapezium Cluster KLF, we are able to derive the underlying Trapezium Cluster IMF, spanning the entire mass range from OB stars to sub-stellar objects near the deuterium burning limit. We find that the stellar Trapezium IMF first rises steeply with a Salpeter-like power-law slope from high mass stars to near $0.6 \mathrm{M}_{\odot}$ where the IMF flattens and forms a broad peak extending to the hydrogen burning limit. There the IMF turns over and declines into the brown dwarf regime. From our modeling experiments in MLL2000, we knew that where an underlying IMF has a power-law form, the young cluster's model KLF also has a power-law form. Further we found that peaks in the model KLFs can arise both due to peaks in the underlying IMF and from features in the M-L relation. From our current modeling of the Trapezium KLF, we find that these conclusions about the relationship between the structure of the KLF and IMF are unchanged by the presence of source reddening. The power-law slope of the bright end $(\mathrm{K}<11.5)$ of the cluster KLF reflects the power-law slope of the derived IMF. The formation of the primary KLF peak is also similar to the structure of the underlying IMF we derive. The broad main peak of the Trapezium KLF is formed by a combination of a peak in the underlying stellar IMF and a feature in the theoretical mass-luminosity relation due to deuterium burning. Moreover, we find that our KLF modeling has allowed us to disentangle these two effects. The main KLF peak at $\mathrm{K}=11-11.5$ corresponds to PMS stars between 0.4 and $0.2 \mathrm{M}_{\odot}$, which according to the DM97 PMS models are undergoing deuterium burning at the mean age of this cluster, while the derived IMF has a broad peak at somewhat lower masses $\left(0.2-0.08 \mathrm{M}_{\odot}\right)$ than the KLF peak would to first order imply. Lastly, our detailed KLF modeling has determined that the turn-over and decline in the cluster KLF does reflect a similar turn-over and decline of the Trapezium IMF across the hydrogen burning limit and is not solely a product of the deuterium burning spike (e.g., Zinnecker, McCaughrean \& Wilking 1993).

\subsubsection{The Sub-Stellar Regime}

As in our work in MLL2000, our KLF modeling technique has permitted us to derive the Trapezium sub-stellar IMF, while the improved depth of our IR census has allowed us to extend this derivation from $0.03 \mathrm{M}_{\odot}$ down to near the deuterium burning limit. Our KLF modeling that now includes source reddening confirms that the steady decline of the cluster KLF between $\mathrm{K}=12$ and $\mathrm{K}=15$ reflects a steady power-law decline in the sub-stellar IMF. Independent of our modeling 
results, however, no more than $22_{-2}^{+4} \%$ of the sources are sub-stellar objects ${ }^{5}$.

The secondary peak in the brown dwarf regime of the cluster KLF at K=15.5 and the subsequent rapid decline of the cluster KLF, however, do not correspond to any known features in the theoretical mass-luminosity relations we have examined (see section 4.2 and figure 14). Moreover, detailed KLF modeling using two different sets of PMS tracks require both the presence of a break from a single power-law decline of the Trapezium brown dwarf IMF around $0.02-0.03 \mathrm{M}_{\odot}$ and the formation of a significant, secondary IMF peak. Using the Burrows et al., (1997) tracks, this IMF peak is located near the deuterium burning limit, 13-14 $\mathrm{M}_{\text {Jup }}$, and is followed by a rapid decline to lower masses. Although both sets of PMS tracks suggest the presence of a secondary peak, the precise details (e.g., location and amplitude) may be track dependent. For example, in the Trapezium sub-stellar IMF found using the B97 tracks, 36\% of the brown dwarfs in the cluster are found in the secondary IMF peak while $64 \%$ have their mass distribution governed by the power-law regime. For the IMF found using our standard merged tracks, these number are 15 and $85 \%$, respectively, however the truncation of the tracks at the lowest masses will slightly skew these latter percentages.

One proviso to the derivation of a significant IMF peak at the deuterium burning limit is the contamination of our IR census by non-cluster members. Though, we have accounted for the background field stars contribution to the cluster KLF, we have also shown that there is reasonable uncertainty in the cloud extinction properties. Additionally, the large beamsize of the $\mathrm{C}^{18} \mathrm{O}$ map may mask low extinction holes in the molecular cloud. Since there are $\sim 75$ sources in the secondary peak of the $\mathrm{M}-\mathrm{A}_{V}$ limited KLF before background subtraction, our current background field star estimate would have to be off more than a factor of two to remove any feature from the IMF at these low masses; it would have to be off by a factor of 4 , however, to account for all of the brown dwarf members. Alternately, our IR census may be contaminated by the presence of low mass members from the intervening but only slightly older Orion OB1c association. Though spectroscopic follow up of a few of these faint sources would separate out background stars (and provide a good test of the $\mathrm{C}^{18} \mathrm{O} \rightarrow \mathrm{A}_{V}$ conversion), members of the foreground $\mathrm{OB} 1$ association would be difficult to spectroscopically separate from actual Trapezium cluster members. However, as we have shown, the derived turn down in the "sub-brown dwarf" IMF below the deuterium burning limit appears independent of background correction.

We conclude, therefore, that if the mass-luminosity relation for low mass brown dwarfs is reasonably robust and does not contain a previously unidentified feature, and our estimate of the contamination of our infrared census by non-cluster members is accurate, then the existing structure of the faint cluster KLF can only be created by a break from a single declining power-law

\footnotetext{
${ }^{5}$ Error based upon the uncertainty in the hydrogen burning limit due to distance and to cluster mean age.
} 
brown dwarf IMF, the formation of a corresponding peak in the underlying Trapezium IMF near the deuterium burning limit, and a rapid decline of the IMF into the planetary mass regime.

\subsection{Sensitivity of Results to Theoretical PMS Models}

The accuracy of an IMF derived for a young stellar cluster is intrinsically dependent upon the robustness of the conversion from observables to a mass function (or individual masses) provided by the theoretical evolutionary models. In MLL2000, we came to the somewhat surprising conclusion that model KLFs were fairly insensitive to differences in the evolutionary PMS models from which the mass-luminosity relations were drawn. This was despite that fact that the detailed physics (e.g., opacities, model atmospheres, internal convection theory, and initial conditions) involved with calculating the theoretical PMS evolutionary models are poorly constrained and that changes in the assumed physics of these models have been shown to produce significant differences in the locations of evolutionary tracks and isochrones on the theoretical HR diagram (D'Antona \& Mazzitelli 1994, 1997; Baraffe et al., 1998; D’Antona 1998; Siess, Dufour \& Forestini 2000; Baraffe et al., 2002). Our findings in MLL2000 would also appear to disagree with recent summaries of the IMF in young clusters which concluded, based upon the track variations in the HR diagram, that the accuracy of current PMS models are the primary uncertainty to the form of the derived IMF (Meyer et al., 2000). Therefore, we explore in more detail the dependence of the theoretical mass-luminosity relation relevant for luminosity function modeling upon the different PMS tracks.

In figure 14 we compare the theoretical mass-infrared luminosity ( $\mathrm{K}$ magnitude) relations converted from six sets of theoretical PMS models for a progressive series of young cluster mean ages. In table 3 we summarize the different input physics and parameters used by various PMS models. The sets of theoretical PMS models were taken from literature sources and converted to observables using a single set of bolometric corrections (see MLL2000). Remarkably, the theoretical mass-K magnitude relations are fairly degenerate between the different PMS models, and those differences that do exist are the largest at very young ages $(\tau<1$ Myrs), agreeing with the recent analysis of (Baraffe et al., 2002). Consequently, this implies that for the Trapezium Cluster there will be some uncertainty in our derived mass function due to the PMS tracks. On the other hand, while the models of Burrows et al., (1997) and Siess, Dufour \& Forestini (2000) display the most significant variations in their predicted M-L relations, we have shown in section 3.3.2 that the sub-stellar Trapezium IMF derived from KLF modeling using the B97 tracks is not significantly different than that found using the DM97 tracks. As we concluded in MLL2000, most differences in the mass-luminosity relations due to differences in input physics are much smaller than we could ever observe and will not impact our modeling results. This result may be understood by 
considering the fact that the luminosity of a PMS star is determined by very basic physics, simply the conversion of gravitational potential energy to radiant luminosity during the Kevin-Helmholtz contraction. And this primarily depends on the general physical conditions in the stellar interior (e.g., whether the interior is radiative or partially to fully convective). The luminosity evolution at the youngest ages ( $<1$ Myrs) will depend, however, on the initial conditions of the contracting PMS star as it leaves its proto-stellar stage, though these differences are quickly erased (Baraffe et al., 2002).

This is significantly different than the situation for mass functions derived by placing the stars on the theoretical HR diagram using spectroscopic and photometric observations. Because most young stars have late type K-M spectral types, they are on nearly vertical Hayashi contraction tracks in the HR diagram. As a result a star's mass derived from the HR diagram is primarily a function of its assigned effective temperature, i.e., its observed spectral type. We illustrate this dependence in figure 15(a) where we plot the predicted effective temperatures as a function of mass for stars in a 1 Myr old cluster. In contrast to the quite similar mass-luminosity relations, a star's mass derived based upon its spectral type is uncertain due to differences in the PMS models by factors of 3 (or more). The conversion from spectral type to mass is made worse by the uncertain conversion of spectral type to effective temperatures for late type sources, resulting from their subgiant gravities (Luhman 1999). Such uncertainties will undoubtedly result in spectroscopically derived IMFs that vary substantially as a function of PMS tracks used (compare, for example, the Luhman et al., 2000, derivation of the Trapezium IMF from DM97 and BCAH98 tracks). Further, these track differences, while decreasing with time, are not resolved by 5 Myrs as shown in figure 15(b). In summary, the uncertainties in the PMS models primarily manifest themselves in variations in the predicted effective temperatures of the young stars rather than the predicted luminosities.

We do not surmise that luminosity function modeling, which employs mass-luminosity relations, is free from systematic dependencies. As concluded in MLL2000, a cluster's mean age must be known in order to derive a cluster's initial mass function from its luminosity function; this can only be derived from placing the stars on the theoretical HR diagram. In general, however, age is a function of luminosity for low mass stars on the HR diagram and will be more or less similar when derived from these PMS models. The exception again occurs at the youngest ages, where the definition of a star's age may differ if the models include the proto-stellar lifetimes. Even in the case of the Palla \& Stahler (1999, hereafter, PS99) models, which begin as protostars accreting along an initial mass-radius relationship or birthline in the HR diagram (Stahler 1983), the massluminosity relations are not substantially divergent from canonical theoretical models except at the very youngest ages. 


\subsection{Comparison of Trapezium IMFs Based on Infrared Observations}

In addition to our initial modeling in MLL2000 and our present study, a number of other authors have recently derived Trapezium IMFs based upon deep infrared observations. While all of these derivations make use of the same set of theoretical pre-main sequence models for converting observations to mass (functions), they use somewhat different cluster parameters and employ a variety of different methodologies. Systematic uncertainties might arise due to varying of cluster parameters such as distance, due to different assumptions about the cluster population such as field star contamination or from simple observational effects such as survey area or the wavelength regime analyzed. Further, it is not understood how closely different methods can arrive at the same IMF.

In figure 16 we compare the IMFs derived by MLL2000, LR2000, HC2000 and Luhman et al., (2000). Globally, these IMFs are remarkably similar. They all have Salpeter-like high mass slopes, all reach a broad peak at sub-solar masses and all decline in frequency with decreasing mass below the hydrogen burning limit with brown dwarf IMF slopes between +1 and +0.5 . After inspecting the different methods and cluster parameters used by these authors, which we summarize in table 4 , this result should be in part expected. When different methods use the same PMS tracks (in this case DM97; $\mathrm{M}<1 \mathrm{M}_{\odot}$ ) and essentially the same star forming histories, the resulting IMFs should basically agree.

The cluster parameters used by these workers are not exactly homogeneous. Further, there are slight variations between these IMFs that might be due in part to observational effects. For example, the truncation or turn down in the high mass end of the LR2000 and HC2000 IMFs is due to bright source saturation in these surveys, not to a real IMF feature. At the low mass end, the IMF derivations appear to diverge below $30 \mathrm{M}_{\text {Jup }}(-1.5$ in log solar mass units) with a "spike" in the LR2000 IMF but no feature in the Luhman et al study. Because LR2000 surveys the largest area while the Luhman et al., (2000) study surveys the smallest area, one might suspect that this difference is due to an increase in field star contamination or, perhaps, counting statistics for the smaller study. The latter is the most likely explanation since both our study and that of HC2000 survey similar large areas and apply field star corrections while finding sub-stellar IMFs that contain either a secondary peak or a plateau at the lowest masses.

Lastly, the methodologies employed range from a purely statistical approach (e.g., the LF modeling of MLL2000 and this paper) to the derivation of individual masses of the stars via a hybrid combination of spectroscopy and infrared colors (e.g., Luhman et al.). It is unclear how to make detailed comparisons of these methods, however, in general, the LR2000, HC2000 and MLL2000 primarily depend upon the theoretical mass-luminosity relation extracted from the PMS tracks. The stellar portion of the Luhman et al IMF depends upon the theoretical HR diagram, while the sub-stellar depends upon the predicted infrared colors and magnitudes. One apparent 
difference between the resulting IMFs that might be related to the different methods is the exact location of the IMF's "peak," or what is sometimes termed the "characteristic" mass. This "peak"

mass varies between IMF derivations by $0.7 \mathrm{dex}$ in log solar mass units. It is not immediately apparent that internal uncertainties in the IMF derivations could cause this scatter. For example, the "peak" of the MLL2000 IMF is revised only 0.1 dex by the inclusions of source reddening. For methods that depend upon mass-luminosity relations, the resulting IMF will be dependent upon the assumed cluster distance and age; modest changes in these parameters should result in slightly different M-L relations and slightly different IMFs. However, there is no strict correlation between "peak" mass and the cluster age or distance used. Hence, we conclude that specific IMF details such as the exact location of "peak" mass cannot be securely identified by these methods; although, we can conclude that the Trapezium IMF peaks at sub-solar masses somewhere between $0.3 \mathrm{M}_{\odot}$ and the hydrogen burning limit.

\subsection{The Trapezium and the Global IMF}

From our current work and by comparison of our work to that of other authors, the general form of the Trapezium IMF is readily apparent: a continuous IMF that rises with a relatively steep slope toward sub-solar masses, flattens and forms a broad peak between $0.3 \mathrm{M}_{\odot}$ and the hydrogen burning limit before turning over and declining into the brown dwarf regime. This IMF structure is roughly half-gaussian, though, not exactly log-normal (see figure 11b ), and it is quite consistent with current derivations of other star cluster IMFs. Open cluster IMFs such as the Pleiades (Bouvier et al., 1998) and M35 (Barrado y Navascués et al., 2001) rise with similar power-law slopes and form broad peaks at sub-solar masses before apparently rolling over and declining into the brown dwarf regime. The color-magnitude diagrams of the very luminous clusters NGC 2362 (Moitinho et al. 2001), NGC 3603 (Brandl et al., 1999), and NGC 6231 (Baume, Vázquez \& Feinstein 1999) all display evidence of IMFs that peak at sub-solar masses, though more complete discussion awaits detailed derivations of their sub-solar and sub-stellar IMFs. Further, this apparent general form of the IMF has also been found for the IMFs of globular clusters (Paresce \& De Marchi 1999) and for the field star IMF (Miller \& Scalo 1979; Kroupa, Tout \& Gilmore 1993; Scalo 1998; Kroupa 2001), though again, the sub-stellar IMFs are not yet robustly known. The continuity of the IMF across so many environments suggests that a single star formation process may be responsible for producing the majority of the mass spectrum. Indeed, evidence that stars and brown dwarfs form with similar initial frequencies of circumstellar disks (Muench et al., 2001) and similar disk properties (Natta \& Testi 2001) suggests that even sub-stellar objects form via the same mechanism as stars. While various physical processes might influence the fine details (the IMF's "peak" mass, for example) of the IMF's final form ( e.g., Adams \& Fatuzzo 1996), the original fragmentation distribution function of a turbulent molecular cloud probably dominates the final form of the stellar 
IMF (Klessen 2001).

It is not clear, however, the extent to which this documented continuity between the Trapezium, open cluster and field star IMFs extends across the entire brown dwarf regime. Very young stellar clusters appear to have consistent low mass IMFs, i.e., IMFs that decline across the hydrogen burning limit (Najita, Tiede, \& Carr 2000; Luhman et al., 2000). As we have already discussed, LR2000 and our work here find that there is reasonable evidence in the Trapezium luminosity function(s) that the brown dwarf Trapezium IMF departs at the lowest masses (e.g., $<30 \mathrm{M}_{\text {Jup }}$; see section 4.3) from a single power-law decline for the brown dwarf regime and forms a strong secondary peak in the IMF near the deuterium burning limit. While a similar IMF feature has yet to be identified in the sub-stellar regime of the well studied IC 348 young cluster (see Najita, Tiede, \& Carr 2000), the recent work of Béjar et al., (2001) suggests a mass function for the young $\sigma$ Orionis cluster (Walter et al., 1997) that is slowly falling throughout the brown dwarf regime but may rise toward the deuterium burning limit.

Were a secondary peak in the IMF of the lowest mass brown dwarfs confirmed, then it may provide evidence for a secondary, competing formation mechanism for these low mass objects. Indeed, the transition in the sub-stellar IMF at $30 \mathrm{M}_{u p}$ from a steady power-law decline to the secondary peak at the deuterium burning limit may represent the transition from the formation of brown dwarfs as individual fragments of the molecular cloud to their formation, for example, as truncated stellar embryos that were dynamically ejected from hierarchical proto-stellar systems before they had a chance to accrete into higher mass objects (Reipurth \& Clarke 2001). Yet the subsequent rapid decline in the IMF below the deuterium burning limit constrains the mass range over which this secondary mechanism is operating. The frequency and characteristics of circumstellar disks around these very low mass brown dwarfs and planetary mass objects may provide an essential test of their formation from individual pre-sub-stellar cores or via some an entirely different mechanism.

\section{Conclusions}

Using a new and very complete near-infrared census of the Trapezium Cluster, we have performed a detailed analysis of the Trapezium Cluster's K band luminosity function and its underlying mass function. Following our earlier work in Muench, Lada \& Lada (2000), we expanded our luminosity function modeling to include the effects of source reddening, and we studied in detail the field star contribution to the cluster KLF. We applied our new models to the Trapezium KLF to

explore its structure and to derive the cluster's initial mass function. From this analysis we draw the following conclusion(s): 
1. The Trapezium Cluster IMF rises in number with decreasing mass and forms a broad peak at sub-solar masses between $0.3 \mathrm{M}_{\odot}$ and the hydrogen burning limit before declining into the brown dwarf regime. Independent of modeling details, no more than $\sim 22 \%$ of the young sources fall below the hydrogen burning limit, placing a strict limit on the brown dwarf population in this cluster.

2. The Trapezium Cluster sub-stellar IMF breaks from a single declining power-law slope between $0.02-0.03 \mathrm{M}_{\odot}$ and forms a significant secondary peak near the deuterium burning limit $\left(\sim 13 \mathrm{M}_{\text {Jup }}\right)$. We derive these results through detailed analysis of the likely field star contamination and from our modeling of the cluster's faint KLF using two different sets of theoretical mass-luminosity relations, although the precise details of this peak do depend upon the PMS models. Regardless, this peak may contain between 15 and $36 \%$ of all the sub-stellar objects in this cluster. Below this peak the sub-stellar IMF declines rapidly toward lower masses suggesting that the yield of freely floating, planetary mass objects during the formation of the Trapezium Cluster was extremely low.

3. We find that source reddening (due to infrared excess and extinction) has only modest effects upon our modeling of the Trapezium cluster's luminosity function. Source reddening tends to broaden the IMFs derived and blur the precision with which we can derive IMF parameters. However, the Trapezium IMF we derive here after accounting for source reddening and field stars is not a substantial revision over that Trapezium IMF we derived in MLL2000.

4. Pre-main sequence luminosity evolution and the resulting age dependent mass-luminosity relations are relatively robust results of most modern PMS evolutionary models, except at the very youngest ages where the models are affected by initial conditions. Conversely, the predicted effective temperatures, hence predicted spectral types, are considerably less robust. This suggests that modeling a cluster's $\mathrm{K}$ band luminosity function is likely to produce a faithful representation of the true IMF of the cluster. Further, we find that the different published methodologies used for deriving the Trapezium IMF from near-infrared photometry produce nearly identical results, although the precise location of a "peak" or characteristic mass for the Trapezium cannot be securely identified.

5. The globally consistent form of the stellar IMF suggests a single physical mechanism may dominate the star formation process in galactic clusters. If the secondary peak in the Trapezium Cluster KLF and the corresponding strong secondary peak in the sub-stellar IMF near the deuterium burning limit were proven to be real cluster characteristics, then this implies that a secondary physical mechanism may be responsible for the formation of very low mass brown dwarfs. Whether this secondary mechanism could be the ejection of very low mass $\left(10-20 \mathrm{M}_{J_{u p}}\right)$ brown dwarfs from hierarchical proto-stellar systems, (e.g., Reipurth \& Clarke), or some other process remains unclear. 
We would particularly like to thank Alyssa Goodman, Kevin Luhman and John Stauffer for helpful discussions, and Pavel Kroupa for especially productive suggestions regarding the modeling algorithms. We acknowledge Ted Bergin for providing the $\mathrm{C}^{18} \mathrm{O}$ and dust continuim maps of the Trapezium Region and thank the anonymous referee for making a number of suggestions that improved the results of the paper. AAM was supported by a Smithsonian Predoctoral Fellowship and by the NASA Graduate Student Research Program (grant NTG5-50233). EAL and AAM acknowledge support from a Research Corporation Innovation Award and Presidential Early Career Award for Scientists and Engineers (NSF AST 9733367) to the University of Florida. This publication makes use of data products from the Two Micron All Sky Survey, which is a joint project of the University of Massachusetts and the Infrared Processing and Analysis Center/California Institute of Technology, funded by the National Aeronautics and Space Administration and the National Science Foundation.

\section{A. FLWO-NTT Near-Infrared Catalog of the Trapezium Cluster}

We summarize in table 5 the characteristics of the three observing runs used to obtain the infrared photometry that comprise the FLWO-NTT Near-Infrared Catalog of the Trapezium Cluster. We compare the area(s) covered by the FLWO-NTT catalog to those of other recent IR surveys in figure 17. The reduction and analysis of the 1997-1998 FLWO observations are fully summarized in Lada et al., (2000), and we do not discuss these further here. Preliminary analysis of the NTT observations was presented in Muench et al., (2001), and we detail these observations and their reduction below.

\section{A.1. NTT 3.5m JHK observations}

Our NTT images of the Trapezium Cluster were obtained under conditions of superb seeing ( $\sim 0.5^{\prime \prime}$ FWHM) on 14 March 2000 using the SOFI infrared spectrograph and imaging camera. The NTT telescope uses an active optics platform to achieve ambient seeing and high image quality, and the SOFI camera employs a large format $1024 \times 1024$ pixel Hawaii HgCdTe array. To obtain a single wide field image of the Trapezium Cluster, we configured SOFI to have a $4.95 \times 4.95$ field of view with a plate scale of 0 "' 29 /pixel. Each exposure consisted of 9 separate dithers each randomly falling within $20^{\prime \prime}$ of the observation center. Each individual dither was the co-average of eight 1.2 second exposures, yielding an total effective integration time of 86.4 seconds for each combined image.

We observed the Trapezium Cluster with identical sequential pairs of on and off-cluster 
dithered images. During one hour on 14 March 2000, we obtained four image pairs of the Trapezium Cluster and off-cluster positions. These were, in temporal order, at $\mathrm{K}_{\mathrm{S}}(2.162 \mu \mathrm{m}), \mathrm{H}(1.65 \mu \mathrm{m})$, $\mathrm{J}(1.25 \mu \mathrm{m})$ and again at $\mathrm{K}_{\mathrm{S}}$, and the on-cluster images had FWHM estimates of $0.53^{\prime \prime}, 0.55^{\prime \prime}$, $0.61^{\prime \prime}$ and $0.78^{\prime \prime}$. Seeing estimates of stars in the paired non-nebulous off-cluster image(s) yielded similar if not marginally higher resolution point spread functions. Observations were taken near transit with a very small range of airmass $(1.138<\sec (z)<1.185)$.

Data reduction of the NTT images was performed using routines in the Image Reduction and Analysis Facility (IRAF) and Interactive Data Language (IDL). Our standard data reduction algorithm was described in Lada et al., (2000) for the FLWO images, and it was subsequently used for the NTT images. Simply, individual dithered frames were reduced using sky and flat field images derived from the non-nebulous off-cluster dithered images which were interspersed with the on-cluster images. Each set of reduced dithered frames were then combined using a standard "shift-and- add" technique. While all the FLWO data was linearized after dark-subtraction using a system supplied linearity correction, linearization coefficients were not obtained for the NTT data. "Sky" flat-fields constructed from the NTT images were compared to system flat-fields which are regularly taken and monitored by the NTT staff. While the NTT system flat-fields were found to vary by only 2-3\% over long periods of time, when we compared our sky flat-fields to the system flat-fields, significant small scale variations (5-10\%) were revealed across the array. We concluded this was due to our relatively short NTT integration times which results in poor sampling of the intrinsically non-flat SOFI array. Therefore, we substituted the system supplied flat-fields into our reduction procedure. The high resolution of our NTT images results in moderate under-sampling of the point spread functions; we tested to see if sub-pixel linear reconstruction (drizzling) of our images would improve our data quality. Since our images have only a few dithers (9), the drizzle algorithm did not improve our result over standard integer "shift-and-add."

The 2000 NTT images had FWHM estimates ranging between 1.8 and 2.1 pixels, and these images are therefore marginally under-sampled and not easily suitable for PSF photometry. Further, the SOFI field of view suffers from coma-like geometric distortions on the northern 10-15\% of the array. For these two reasons, we decided to perform only aperture photometry on the NTT images. Multi-aperture photometry was performed on sources detected in the NTT image using annuli with radii from 2 to 10 pixels. The sky was measured from the mode of the distribution of pixel values in an annuli from 10 to 20 pixels. From inspection of the curves of growth of both isolated and nebulous sources, we chose a 3 pixel radius $\left(1.8^{\prime \prime}\right.$ beam) for most of our NTT sources. Additionally, the choice of small apertures allowed us to minimize the effects of nebular contamination and crowding on the stellar PSF. For faint sources in very confused or highly nebulous regions, we repeated the photometry with a 2 pixel aperture and a sky annulus from 7 to 12 pixels. The change in sky annulus does not significantly affect our photometry because the fraction of the stellar PSF beyond 7 pixels contains less than 5\% of the flux, and the errors resulting from 
including this flux in the sky estimate are smaller than the errors introduced from using too distant a sky annulus on the nebulous background.

Aperture corrections were derived for our data by choosing $\sim 15$ relatively bright stars as free of nebular contamination as possible. We performed multi-aperture photometry on them and using the IRAF MKAPFILE routine to visually inspect the stellar curves of growth and calculate corrections. Since small apertures were used to minimize the effects of the bright nebular background, the resulting corrections which constituted a somewhat substantial fraction of the stellar flux. Aperture corrections were carefully checked by comparing the corrections derived for on (nebulous) and off-cluster positions, which are interspersed in time with the on-cluster frames, and found to agree or to correlate with changes in seeing. The typical 3 pixel aperture correction was -0.14 magnitudes and for those stars photometered using a 2 pixel aperture, a correction of -0.34 was used.

\section{A.2. Photometric Comparisons of Datasets}

We report in the electronically published catalog all the photometry from the FLWO and NTT observations. We explored any photometric differences between the FLWO and NTT observations. These differences include the filter systems, the methods and effective beamsizes of the photometry and the epochs of the observations. We tested if any color terms were present due to differing photometric (filter) systems, we compared the magnitudes and colors of 504 sources common to both the NTT and FLWO photometry.We compared the $(\mathrm{J}-\mathrm{H})$ and $(\mathrm{H}-\mathrm{K})$ colors of the NTT photometry to the FLWO photometry and fit these comparisons with linear relations. The $(\mathrm{J}-\mathrm{H})$ colors were well fit by a linear relation (slope $\sim 1$ ); however, we found an offset, $\Delta(J-H) \simeq 0.10$ magnitudes between the two systems. A similar comparison to the photometry of sources in the 2MASS catalog ${ }^{6}$ indicated this offset was at $\mathrm{J}$ band and was restricted to the FLWO sources. Comparison of 2MASS photometry to the NTT photometry revealed no systematic offsets. A comparison of the FLWO and NTT $(\mathrm{H}-\mathrm{K}$ ) colors was also well fit by a linear relation (slope $\sim$ $0.97)$ though this slope suggests that for the reddest sources, the NTT $\left(\mathrm{H}-\mathrm{K}_{\mathrm{S}}\right)$ color is bluer than the FLWO (H - K) color.

Further, it was evident from these comparisons that while the global filter systems are quite similar, the difference in the NTT and FLWO photometry of individual sources was larger than expected from formal photometric errors ${ }^{7}$. From our fake star experiments and from the photometry

\footnotetext{
${ }^{6}$ A current un-restricted search of the 2MASS First and Second Incremental Point Source and Extended Source Catalogs currently returns only 171 sources.

${ }^{7}$ the quadratic sum of uncertainties from aperture corrections, zeropoint and airmass corrections, flat fielding error
} 
of sources in overlap regions on mosaicked frames, we determined our measured photometric error is $5 \%$ for the majority of our sources increasing up to $15 \%$ for the sources at our completeness limit. However, when comparing sources common to both the FLWO and NTT data (well above our completeness limit), we derived $1 \sigma$ standard deviations of $\sim 0.22$ for magnitudes and $\sim 0.18$ for colors. Very similar dispersions were derived when comparing our FLWO photometry to the Hillenbrand et al., (1998) or 2MASS catalogs or when comparing our NTT data to the Hillenbrand \& Carpenter (2000) $\mathrm{H}$ and $\mathrm{K}$ band dataset. We attribute a portion of this additional photometric noise between the different datasets to the intrinsic infrared variability of these pre-main sequence sources which has been found for stars in this cloud to have an average of 0.2 magnitudes at infrared wavelengths (Carpenter, Hillenbrand \& Strutskie 2001). We note that the difference in the beamsize used for the FLWO and NTT photometry and by the various other published data sets will also contribute a degree of added photometric noise due to the presence of the strong nebular background, thus making the NTT photometry preferable to the FLWO data for its higher angular resolution.

\section{A.3. Astrometry and the Electronic Catalog}

Astrometry with reasonably high precision was performed by matching the XY pixel locations of a large number $(>50$ ) of the observed sources to the equatorial positions of these sources listed on the 2MASS world coordinate system and deriving full plate solutions using the IRAF CCMAP routine. Mosaic positions of the 1997 and 1998 observations were shifted to fall onto a common XY pixel grid defined by the K band FLWO 1997 mosaic images. To create the common K band $\mathrm{XY}$ grid, sources in the overlap regions between mosaic positions were matched and global offsets calculated. The two camera arrays of the FLWO STELIRcam instrument are not centered precisely on the sky and the $\mathrm{J}$ and $\mathrm{H}$ band coordinates were transformed using the IRAF GEOMAP routine into the K band XY coordinate grid. The NTT positions were aligned to the NTT J band image. For the FLWO plate solution, 161 2MASS sources were matched to the FLWO XY coordinates yielding a plate scale of 0.596 "/pixel and an astrometric solution with rms errors of $\sim 0.10^{\prime \prime}$. An independent solution of 82 NTT sources matched to the 2MASS database yielded a plate scale of $0.288 \% /$ pixel and an astrometric solution having rms errors $\sim 0.07^{\prime \prime}$.

We construct the electronic version of the FLWO-NTT catalog based upon all the sources detected by our FLWO and NTT observations, and we compliment our electronic catalog by including sources identified in other catalogs and falling within our survey area, but that were saturated, undetected or unresolved by our observations. Since our final catalog covers a substantially different

and sky noise 
area than comparable deep infrared surveys and includes numerous new sources, we chose to assign new source designations for our final catalog. These are based upon the IAU standard format that includes a catalog acronym, a source sequence, and source specifier. For the catalog acronym, we chose the MLLA, based upon the initials of the last names of the authors. This acronym is currently unused in the Dictionary of Celestial Nomenclature. We chose to sequence the catalog using a running number incremented from 00001 to 01010 . We use a specifier only where necessary to distinguish unresolved sources, typically employing the designations (A), (B), etc. NTT astrometry is preferentially used in the final catalog. For undetected or unresolved sources, we made every effort to include astrometry from the source's identifying catalog if the original catalog could be globally aligned to the FLWO-NTT catalog. We list cross-references based on the most comprehensive or deep surveys; these include the Hillenbrand (1997), HC2000, Luhman et al., (2000) designations and McCaughrean \& Stauffer (1994). For sources lacking cross-references in these catalogs, we list their 2MASS designations (circa the 2nd Incremental 2MASS Point Souce Catalog) where possible. The LR2000 designations are based on their derived equatorial coordinates and due to significant astrometric errors do not correspond to the positions we derive in the FLWO-NTT catalog. For example, we find off-sets of $-0.42^{\prime \prime}$ in RA and $0.44^{\prime \prime}$ in DEC between our positions and those of LR2000. After removing these offsets, we still find medain residuals of $0.44^{\prime \prime}$ between our coordinates and those of LR2000 with errors as large as $1^{\prime \prime}$; this is in contrast to the rms residuals of $0.1^{\prime \prime}$ between our catalog and the 2MASS and HC2000 positions. Hence we do not list the LR2000 position-dependent designations except where necessary to identify sources undetected by our catalog. 


\section{REFERENCES}

Adams, F.C., \& Fatuzzo, M. 1996, ApJ, 464, 256

Alexander, D.R., Augason, G.C., \& Johnson, H.R. 1989, ApJ, 345, 1014

Alexander, D.R., \& Ferguson, 1994, ApJ, 437, 879

Alves, J., Lada, C.J., Lada, E.A., Kenyon, S.J., \& Phelps, R. 1998, ApJ, 506, 292

Alves, J., Lada, C.J., \& Lada, E.A. 1999, ApJ, 515, 265

Barrado y Navascués, D., Stauffer, J.R., Bouvier, J., \& Martín, E.L. 2001, ApJ, 546, 1006

Baume, G., Vázquez, R.A., \& Feinstein, A. 1999, A\&AS, 137, 233

Baraffe, I., Chabrier, G., Allard, F., \& Hauschildt, P.H. 1998, A\&A, 337, 403 (BCAH98)

Baraffe, I., Chabrier, G., Allard, F., \& Hauschildt, P.H. 2002, A\&A, in press (BCAH02)

Béjar, V.J.S., Martín, E.L., Zapatero Osorio, M.R., Rebolo, R., Barrado y Navascués, D., BailerJones, C.A.L., Mundt, R., Baraffe, I., Chabrier, C., \& Allard, F. 2001, ApJ, 556, 830

Bernasconi, P.A. 1996, A\&AS, 120, 57

Bessell, M. S. 1995, Proceedings of the ESO Workshop: The Bottom of the Main Sequence - and Beyond, ed C. G. Tinney, (Heidelberg: Springer), 123

Bouvier, J., Stauffer, J.R., Martín, E.L., Barrado y Navascués, D., Wallace, B., \& Bejar, V.J.S. 1998, A\&A, 336, 490

Brandl, B., Brandner, W., Eisenhauer, F., Moffat, A.F.J., Palla, F., \& Zinnecker, H. 1999, A\&A, 352,69

Burrows, A., Marley, M., Hubbard, W.B., Lunine, J.I., Guillot, T., Saumon, D., Freedman, R., Sudarsky, D., \& Sharp, C. 1997, ApJ, 491, 856 (B97)

Canuto, V.M., \& Mazzitelli, I. 1991, ApJ, 370, 295

Canuto, V.M., \& Mazzitelli, I. 1992, ApJ, 389, 724

Canuto, V.M., Goldman, I., \& Mazzitelli, I. 1996, ApJ, 473, 550

Carpenter, J.M., Hillenbrand, L.A., \& Strutskie, M.F. 2001, AJ, 121, 3160

Cohen, J.G., Persson, S.E., Elias, J.H., \& Frogel, J.A. 1981, ApJ, 249, 481 
D’Antona, F., \& Mazzitelli, I. 1994, ApJS, 41, 467

D’Antona, F., \& Mazzitelli, I. 1997, Cool Stars in Clusters and Associations, ed G. Micela and R. Pallavicini, (Mem. S.A.It.), 68, 807

D’Antona, F. 1998, 38th Herstmonceux Conference: The Stellar Initial Mass Function, ed G. Gilmore \& D. Howell, (San Francisco: A.S.P), 142, 157

Frerking, M.A., Langer, W.D., \& Wilson, R.W. 1982, ApJ, 262, 590

Gilmore, G. 2001, Starbursts: Near and Far, eds. L.J. Tacconi \& D. Lutz, in press

Goldsmith, P.F., Bergin, E.A., \& Lis, D.C. 1997, ApJ, 491, 615

Hauschildt, P.H., Allard, F., \& Baron, E. 1999, ApJ, 512, 377

Hillenbrand, L.A. 1997, AJ, 113, 1733

Hillenbrand, L.A., Strom, S.E., Calvet, N., Merrill, K.M., Gatley, I., Makidon, R., Meyer, M.R., \& Skrutskie, M.F. 1998, AJ, 116, 1816

Hillenbrand, L.A., \& Carpenter, J.M. 2000, ApJ, 540, 236 (HC2000)

Iglesias, C.A., \& Rogers, F.J. 1996, ApJ, 464, 943

Kenyon, S.J., \& Hartmann, L.W. 1995, ApJS, 101, 117

Klessen, R.S. 2001, ApJ, 556, 837

Kroupa, P., Tout, C.A., \& Gilmore, G. 1993, MNRAS, 262, 545

Kroupa, P. 2001, MNRAS, 322, 231

Lada, C.J., Muench, A.A., Haisch, K.J., Lada, E.A., Alves, J., Tollestrup, E.V., \& Willner, S.P. 2000, AJ, 120, 3162

Lucas, P.W., \& Roche, P.F. 2000, MNRAS, 314, 858 (LR2000)

Luhman, K.L., Rieke, G.H., Lada, C.J., \& Lada, E.A. 1998, ApJ, 508, 347

Luhman, K.L., \& Rieke, G.H. 1999, ApJ, 525, 440

Luhman, K.L. 1999, ApJ, 525, 466

Luhman, K.L., Reike, G.H., Young, E.T., Cotera, A.S., Chen, H., Rieke, M.J., Schneider, G., \& Thompson, R.I. 2000, AJ, 540, 1016 
McCaughrean, M.J., \& Stauffer, J.R. 1994, AJ, 108, 1382

McCaughrean, M., Zinnecker, H., Rayner, J., \& Stauffer, J. 1995, Proceedings of the ESO Workshop: The Bottom of the Main Sequence - and Beyond, ed C. G. Tinney, Springer: Heidelberg, 207

Meyer, M.R., Calvet, N., \& Hillenbrand, L.A. 1997, AJ, 114, 233

Meyer, M.R., Adams, F.C., Hillenbrand, L.A., Carpenter, J.M., \& Larson, R.B. 2000, Protostars and Planets IV, ed V. Mannings, A.P. Boss, and S.S. Russell, (Tucson: Univer. of Arizona), 121

Mihalas, D., Däppen, W., \& Hummer, D.G. 1988, ApJ, 331, 815 (MHD)

Miller, G.E., \& Scalo, J.M. 1979, ApJS, 41, 513

Moitinho, A., Alves, J., Huélamo, N., \& Lada, C.J. 2001, ApJ, 563, 73

Muench, A.A., Lada, E.A., \& Lada, C.J. 2000, ApJ, 533, 358 (MLL2000)

Muench, A.A., Alves, J., Lada, C.J., \& Lada, E.A. 2001, ApJ, 558, 51

Najita, J., Tiede, G., \& Carr, J. 2000, ApJ, 541, 977

Natta, A., \& Testi, L. 2001, A\&A, 376, 22

Palla, F., \& Stahler, S.W. 1999, AJ, 525, 772 (PS99)

Paresce, F., \& De Marchi, G. 1999, ApJ, 534870

Pols, O.R., Tout, C.A., Eggleton, P.P., \& Han, Z. 1995, MNRAS, 274, 964 (PTEH)

Reipurth, B., \& Clarke, C. 2001, AJ, 122, 432

Rogers, F.J., \& Iglesias, C.A. 1992, ApJ, 401, 361 (OPAL92)

Rogers, F.J., Swenson, F.J, \& Iglesias, C.A. 1996, ApJ, 456, 902 (OPAL96)

Salpeter, E.E. 1955, ApJ, 121, 161

Saumon, D., \& Chabrier, G. 1991, Phys. Rev. A, 44, 5122 (SC)

Saumon, D., \& Chabrier, G. 1992, Phys. Rev. A, 46, 2084 (SC)

Saumon, D., Chabrier, G., \& VanHorn, H.M. 1995, ApJS, 99, 713 (SCVH) 
Scalo, J. 1998, 38th Herstmonceux Conference: The Stellar Initial Mass Function, ed G. Gilmore and D. Howell, (San Francisco: A.S.P), 201

Schaller, G., Schaerer, D., Meynet, G., \& Maeder, A 1992, A\&AS, 96, 269

Siess, L., Dufour, E., \& Forestini, M. 2000, A\&A, 358, 593 (SDF)

Stahler, S.W. 1983, ApJ, 274, 822

Walter, F.M, Wolk, S.J., Freyberg, M., \& Schmitt, J.H.M.M. 1997 Memorie della Societa Astronomica Italiana, 68, 1081

Wilking, B.A., Greene, T.P., \& Meyer, M.R. 1999, AJ, 117, 469

Zinnecker, H., McCaughrean, M. \& Wilking, B. A. 1993, Protostars and Planets III, ed H. Levy and J.I. Lunine, (Tucson: Univer. of Arizona), 429 
Table 1. Three Power-Law IMF Parameters and Fits ${ }^{(a)}$

\begin{tabular}{|c|c|c|c|c|c|}
\hline Parameter $^{(b)}$ & Range of Fits & Best Fit ${ }^{(c)}$ & $( \pm)$ & Best Fit ${ }^{(d)}$ & $( \pm)$ \\
\hline \multicolumn{6}{|c|}{ Model fit to $\mathrm{K}=14.5 ; \quad \chi^{2} \sim 1^{(e)}$} \\
\hline$\Gamma_{1}$ & $-1.0 \longleftrightarrow-2.0$ & -1.16 & 0.16 & -1.24 & 0.20 \\
\hline $\log m_{1}$ & $+0.1 \longleftrightarrow-1.1$ & -0.17 & 0.10 & -0.19 & 0.13 \\
\hline$\Gamma_{2}$ & $-0.4 \longleftrightarrow+0.4$ & -0.24 & 0.07 & -0.16 & 0.15 \\
\hline $\log m_{2}$ & $+0.1 \longleftrightarrow-1.4$ & -1.05 & 0.05 & -1.00 & 0.13 \\
\hline$\Gamma_{3}$ & $-0.4 \longleftrightarrow+2.0$ & 1.10 & 0.25 & 1.08 & 0.38 \\
\hline \multicolumn{6}{|c|}{ Model Fit to $\mathrm{K}=15.0 ; \quad \chi^{2} \sim 1$} \\
\hline$\Gamma_{1}$ & & -1.13 & 0.16 & -1.21 & 0.18 \\
\hline $\log m_{1}$ & & -0.19 & 0.11 & -0.22 & 0.11 \\
\hline$\Gamma_{2}$ & & -0.24 & 0.15 & -0.15 & 0.17 \\
\hline $\log m_{2}$ & & -1.00 & 0.10 & -0.92 & 0.13 \\
\hline$\Gamma_{3}$ & & 0.82 & 0.15 & 0.73 & 0.20 \\
\hline \multicolumn{6}{|c|}{ Model Fit to $\mathrm{K}=15.5 ; \quad \chi^{2} \sim 0.3$} \\
\hline $\log m_{2}$ & & -0.89 & - & -0.77 & 一 \\
\hline$\Gamma_{3}$ & & 0.30 & - & 0.30 & - \\
\hline \multicolumn{6}{|c|}{ Model Fit to $\mathrm{K}=16.5 ; \quad \chi^{2} \sim 0.3$} \\
\hline $\log m_{2}$ & & - & - & -0.72 & - \\
\hline$\Gamma_{3}$ & & - & - & 0.23 & - \\
\hline
\end{tabular}

${ }^{(a)}$ All tabulated fits derived using our standard set of PMS tracks (primarily from DM97).

${ }^{(b)}$ The parameters $\Gamma_{i}$ are the power-law indices of the IMF which here is defined as the number of stars per unit $\log \left(\frac{M}{M_{\odot}}\right)$. The parameters $m_{j}$ are the break masses in the power-law IMF and are in units of $\log \left(\frac{M}{M_{\odot}}\right)$.

${ }^{(c)}$ Model fits without Source Reddening.

${ }^{(d)}$ Model fits accounting for Source Reddening.

${ }^{(e)} \chi^{2}$ given for fits with Source Reddening. 
Table 2. Three Power-Law Trapezium Sub-Stellar IMF ${ }^{(a)}$

\begin{tabular}{|c|c|c|c|c|c|c|}
\hline \multirow{2}{*}{$\begin{array}{l}\text { IMF Parameter } \\
\qquad(\varphi)\end{array}$} & \multicolumn{3}{|c|}{ Fit to $K=16.0$} & \multicolumn{3}{|c|}{ Fit to $K=16.5$} \\
\hline & $<\varphi>$ & $1 \sigma$ & $\operatorname{median}(\varphi)$ & $<\varphi>$ & $1 \sigma$ & $\operatorname{median}(\varphi)$ \\
\hline \multicolumn{7}{|c|}{ Fits to $\mathrm{M}-\mathrm{A}_{V}$ KLF w/o Off-field Correction ${ }^{(b)}$} \\
\hline$\Gamma_{1}$ & +1.39 & 0.22 & $+1,30$ & +1.36 & 0.18 & +1.30 \\
\hline$m_{1}$ & 0.0214 & 0.0022 & 0.020 & 0.0220 & 0.0025 & 0.020 \\
\hline$\Gamma_{2}$ & -4.87 & 1.52 & -5.00 & -5.50 & 2.01 & -5.00 \\
\hline$m_{2}$ & 0.0125 & 0.0012 & 0.012 & 0.0137 & 0.0005 & 0.014 \\
\hline$\Gamma_{3}$ & +3.70 & 2.60 & +4.00 & +5.70 & 1.59 & +6.00 \\
\hline \multicolumn{7}{|c|}{ Fits to $\mathrm{M}-\mathrm{A}_{V}$, Off-field Corrected $\mathrm{KLF}^{(b)}$} \\
\hline$\Gamma_{1}$ & +1.53 & 0.13 & $+1,60$ & +1.51 & 0.14 & +1.60 \\
\hline$m_{1}$ & 0.0217 & 0.0024 & 0.020 & 0.0228 & 0.0025 & 0.025 \\
\hline$\Gamma_{2}$ & -5.43 & 1.95 & -6.00 & -5.04 & 2.00 & -4.00 \\
\hline$m_{2}$ & 0.0130 & 0.0008 & 0.013 & 0.0137 & 0.0008 & 0.014 \\
\hline$\Gamma_{3}$ & +3.87 & 2.47 & +4.00 & +5.61 & 2.25 & +6.00 \\
\hline
\end{tabular}

${ }^{(a)}$ Fits to Sub-Stellar Trapezium KLF using B97 tracks.

${ }^{(b)} \mathrm{M}-\mathrm{A}_{V} \mathrm{KLF}$ has limits of $\mathrm{A}_{V} \leq 9$ and $\mathrm{M} \geq 0.01 \mathrm{M}_{\odot}$ 
Table 3. Summary of Theoretical Pre-Main Sequence Calculations

\begin{tabular}{|c|c|c|c|c|c|c|c|c|c|}
\hline $\begin{array}{l}\text { Model }^{(a)} \\
\text { Name }\end{array}$ & $\begin{array}{c}\tau_{\min }{ }^{(b)} \\
(\mathrm{Myrs})\end{array}$ & $\begin{array}{l}\mathbf{M}_{\max } \\
\left(\mathbf{M}_{\odot}\right)\end{array}$ & $\begin{array}{l}\mathrm{M}_{\min } \\
\left(\mathrm{M}_{\odot}\right)\end{array}$ & $\begin{array}{r}\text { Initial }^{(c)} \\
\text { Conditions }\end{array}$ & {$[\mathrm{D} / \mathrm{H}]^{(d)}$} & $\begin{array}{l}\text { Opacity }{ }^{(e)} \\
\text { Table }\end{array}$ & $\operatorname{EOS}^{(f)}$ & $\begin{array}{l}\text { Convection } \\
\text { Model }\end{array}$ & $\begin{array}{l}\text { Atmosphere } \\
\text { Model }\end{array}$ \\
\hline DM94 & 0.1 & 2.50 & 0.020 & Canonical & 2.0 & OPAL92+Alex89 & MHD & FST-1 & Gray \\
\hline B97 & 0.1 & 0.15 & 0.001 & Canonical & 2.0 & $?$ & $\mathrm{SC}$ & $?$ & Non-Gray \\
\hline DM97 & 0.1 & 3.00 & 0.017 & Canonical & 2.0 & OPAL92+Alex94 & $\mathrm{MHD}+\mathrm{OPAL}$ & FST-2 & Gray \\
\hline BCAH98 & 1.0 & 1.40 & 0.020 & Canonical & 2.0 & OPAL96+Alex94 & $\mathrm{SCVH}$ & MLT-1.0 & Non-Gray(NextGen) \\
\hline PS99 & 0.1 & 6.00 & 0.100 & Birthline & - & OPAL96+Alex94 & PTEH & MLT-1.5 & Gray \\
\hline SDF00 & 0.1 & 7.00 & 0.100 & Canonical & 2.0 & OPAL96+Alex94 & PTEH(r) & MLT-1.6 & Analytic Fit \\
\hline
\end{tabular}

a All models used standard solar metallicity $(\mathrm{Z}=0.02)$.

${ }^{\mathrm{b}}$ Minimum Age (Myrs) listed by the models.

${ }^{\mathrm{c}}$ Initial Physical Conditions from which the tracks are evolved. Canonical: the model stars are evolved from "infinite" spheroids; Birthline: the PS99 models begin with spherically accreting (single accretion rate, $10^{-5} \mathrm{M}_{\odot} y r^{-1}$ ) protostars evolving along the birthline before beginning their PMS contraction phase.

${ }^{\mathrm{d}}$ Deuterium abundance relative to hydrogen, in units of $10^{-5}$. The initial D/H for the PS99 models was $2.0 \times 10^{-5}$, however this is significantly modified by the burning of deuterium during the model's proto-stellar phase.

e Opacity Table (interior not atmosphere): Alex89 (Alexander et al., 1989); OPAL92 (Rogers \& Iglesias 1992); Alex94 (Alexander \& Ferguson 1994); OPAL96 (Iglesias \& Rogers 1996)

${ }^{\mathrm{f}}$ Convection Model: FST (Full Spectrum Turbulence. FST-1: Canuto \& Mazzitelli (1991, 1992), FST-2: Canuto et al., (1996)); MLT-1.X (Mixing Length Theory, $1 . \mathrm{X}=\alpha=1 / \mathrm{H}_{p}$ )

g Equation of State: MHD (Mihalas, Däppen \& Hummer 1988); SC (Saumon \& Chabrier 1991, 1992); SCVH (Saumon, Chabrier \& VanHorn 1995); PTEH (Pols et al., 1995, r: Revised by SDF), OPAL (Rogers, Swenson \& Iglesias 1996

${ }^{\text {h }}$ Treatment of Atmosphere: Analytic Fit (SDF00) is a 1D fit of $T(\tau)$ to atmosphere models; NextGen (Hauschildt, Allard \& Baron 1999) 
Table 4. Comparison of published Trapezium IMFs $\stackrel{(a)}{(a)}$

\begin{tabular}{|c|c|c|c|c|c|c|c|c|c|c|}
\hline $\begin{array}{l}\text { Work } \\
\text { Name }\end{array}$ & $\begin{array}{c}\text { IMF } \\
\text { Method }\end{array}$ & $\begin{array}{c}\text { Distance } \\
(\mathrm{pc}),(\mathrm{m}-\mathrm{M})\end{array}$ & $\begin{array}{l}\tau, \Delta \tau^{(b)} \\
(\mathrm{Myr})\end{array}$ & Extinction? ${ }^{(c)}$ & IR Excess? ${ }^{(c)}$ & $\begin{array}{l}\text { Field Star }{ }^{(c)} \\
\text { Correction? }\end{array}$ & $\mathrm{A}_{V}$ Limit? & $\begin{array}{c}\text { IMF Peak } \\
\left(\frac{M}{M_{\odot}}\right)\end{array}$ & $\begin{array}{l}\operatorname{Area}^{(d)} \\
(\square \mathrm{pc})\end{array}$ & Comments ${ }^{(e)}$ \\
\hline MLL2000 & $\begin{array}{l}\text { Model KLF } \\
\text { Fit }\end{array}$ & $400,8.00$ & $0.8,1.2$ & $\begin{array}{l}\text { Not } \\
\text { Included }\end{array}$ & $\begin{array}{l}\text { Not } \\
\text { Included }\end{array}$ & $\begin{array}{l}\text { Not } \\
\text { Included }\end{array}$ & None & 0.08 & 0.34 & $\begin{array}{r}\text { Literature } \\
\text { K only }\end{array}$ \\
\hline HC2000 & $(\mathrm{H}-\mathrm{K}) / \mathrm{K}$ & $480,8.40$ & $0.4,1.0$ & Derived & Empirical & $\begin{array}{l}\text { Reddened } \\
\text { Galaxy Model }\end{array}$ & $\begin{array}{c}\mathrm{A}_{V}<2.5,10 \\
\text { no limit }\end{array}$ & 0.15 & 0.35 & $\begin{array}{l}\text { Keck } \\
\mathrm{H}, \mathrm{K}\end{array}$ \\
\hline LR2000 & $\mathrm{M}_{J} \rightarrow \mathrm{M}_{\odot}$ & $440,8.22$ & $1.0,0.0$ & Derived & $\begin{array}{l}\text { Assumed } \\
\text { None }\end{array}$ & $\begin{array}{l}\text { Assumed } \\
\text { None }\end{array}$ & $(\mathrm{J}-\mathrm{H})<1.5$ & 0.40 & 0.50 & $\begin{array}{r}\text { UKIRT } \\
\text { I, J, H }\end{array}$ \\
\hline $\begin{array}{l}\text { Luhmen } \\
\text { et al }\end{array}$ & $\begin{array}{l}\text { IR Spectra } \\
+ \text { Colors }\end{array}$ & $450,8.27$ & $0.4, \sim 1$ & Derived & $\begin{array}{l}\text { Assumed } \\
\text { None }\end{array}$ & $\begin{array}{l}\text { Assumed } \\
\text { None }\end{array}$ & $\mathrm{A}_{H}<1.4$ & 0.25 & 0.073 & $\begin{array}{r}\text { NICMOS } \\
\text { F110, F160 }\end{array}$ \\
\hline
\end{tabular}

${ }^{(a)}$ All IMF derivations used the D’Antona \& Mazzitelli (1997) pre-main sequence models for masses less than $1 \mathrm{M}_{\odot}$.

${ }^{(b)}$ Cluster mean age and age spread used by authors. For Luhman et al., (2000), an empirical star forming history was used by the authors and those tabulated here are approximate characterizations.

${ }^{(c)}$ Listed if and how these quantities: extinction, excess and the contribution of background field stars, were included into that work's IMF derivation.

${ }^{(d)}$ Size (area) of surveys in $\square$ parsec assuming D $=400 \mathrm{pc}$. The two values for this work are for the NTT/FLWO overlap region and the larger FLWO region.

${ }^{(e)}$ Comments include location of observation(s) and broadband filters used. 
Table 5. Summary of Near-Infrared Observations

\begin{tabular}{lcccl}
\hline \hline \multicolumn{2}{c}{ Observatory $^{(a)}$} & $\begin{array}{c}\text { Date } \\
\text { YYYY/MM/DD }\end{array}$ & \multicolumn{3}{c}{$\begin{array}{c}\text { Passband } \\
(b)\end{array}$} \\
& Plate Scale $(c)$ & Comments \\
Beamsize & \\
\hline FLWO & $1997 / 12 / 14$ & $\mathrm{H}$ & $0.596 / 3.58$ & see also Lada et al. (2000) \\
FLWO & $1997 / 12 / 14$ & $\mathrm{~K}$ & $0.596 / 3.58$ & \\
FLWO & $1997 / 12 / 15$ & $\mathrm{H}$ & $0.596 / 3.58$ & \\
FLWO & $1997 / 12 / 15$ & $\mathrm{~K}$ & $0.596 / 3.58$ & \\
FLWO & $1997 / 12 / 16$ & $\mathrm{~J}$ & $0.596 / 3.58$ & \\
FLWO & $1998 / 11 / 04$ & $\mathrm{~J}$ & $0.596 / 3.58$ & see also Lada et al. (2000) \\
FLWO & $1998 / 11 / 04$ & $\mathrm{H}$ & $0.596 / 3.58$ & \\
FLWO & $1998 / 11 / 04$ & $\mathrm{~L}$ & $0.596 / 3.58$ & \\
NTT & $2000 / 03 / 14$ & $\mathrm{~K}$ & $0.288 / 1.73$ & see also Muench et al (2001) \\
NTT & $2000 / 03 / 14$ & $\mathrm{H}$ & $0.288 / 1.73$ & \\
NTT & $2000 / 03 / 14$ & $\mathrm{~J}$ & $0.288 / 1.73$ & \\
NTT & $2000 / 03 / 14$ & $\mathrm{~K}$ & $0.288 / 1.73$ & \\
\hline
\end{tabular}

(a) FLWO: Fred Lawrence Whipple Observatory, Smithsonian Astrophysical Observatory; NTT: New Technology Telescope, European Southern Observatory.

${ }^{(b)}$ Filter central wavelengths $\left.\lambda(\mu \mathrm{m})\right)$ : FLWO- J) 1.25 ; H) 1.65 ; K) 2.20 ; L) 3.50 ; . NTT- J) $\left.1.25 ; \mathrm{H}) 1.65 ; \mathrm{K}_{\mathrm{S}}\right) 2.16$.

${ }^{(c)}$ Plate scale: arcsec/pixel; Beamsize: the effective diameter of photometry beam (arcsec) 
Table 6. FLWO-NTT Near-Infrared Catalog

\begin{tabular}{|c|c|c|c|c|c|c|c|c|c|c|c|c|c|c|c|c|c|c|c|c|c|}
\hline \multirow[t]{2}{*}{ Seq. } & \multirow[t]{2}{*}{ Spec. } & \multirow{2}{*}{$\begin{array}{l}\text { R.A. } \\
\text { (J2000) }\end{array}$} & \multirow{2}{*}{$\begin{array}{c}\text { Dec. } \\
\text { (J2000) }\end{array}$} & \multicolumn{4}{|c|}{ FLWO (Mag) } & \multicolumn{4}{|c|}{ FLWO (Err) } & \multicolumn{3}{|c|}{ NTT (Mag) } & \multicolumn{3}{|c|}{ NTT (Err) } & \multirow{2}{*}{$\begin{array}{l}\text { Phot } \\
\text { Flag }\end{array}$} & \multirow{2}{*}{$\begin{array}{c}\text { H97 } \\
\text { ID }\end{array}$} & \multirow{2}{*}{$\begin{array}{l}\text { HC2000 } \\
\text { ID }\end{array}$} & \multirow{2}{*}{$\begin{array}{c}\text { Other } \\
\text { ID }\end{array}$} \\
\hline & & & & $\mathrm{J}$ & $\mathrm{H}$ & $\mathrm{K}$ & $\mathrm{L}$ & $\mathrm{J}$ & $\mathrm{H}$ & $\mathrm{K}$ & $\mathrm{L}$ & $\mathrm{J}$ & $\mathrm{H}$ & $\mathrm{K}_{\mathrm{S}}$ & $\mathrm{J}$ & $\mathrm{H}$ & $\mathrm{K}_{\mathrm{S}}$ & & & & \\
\hline 00001 & & 53522.45 & -52610.9 & 14.59 & 13.68 & 99.00 & 12.40 & 0.02 & 0.06 & -1.00 & 0.26 & & & & & & & 0 & & & \\
\hline 00002 & & 53526.57 & -52609.6 & 16.24 & 15.41 & 99.00 & 99.00 & 0.04 & 0.01 & -1.00 & -1.00 & & & & & & & 0 & & & \\
\hline 00003 & & 53511.65 & -52609.0 & 13.51 & 11.63 & 10.56 & 9.21 & 0.04 & 0.01 & 0.02 & 0.02 & & & & & & & 0 & & & \\
\hline 00004 & & 53515.97 & -52607.4 & 14.12 & 12.25 & 99.00 & 10.30 & 0.02 & 0.01 & -1.00 & 0.04 & & & & & & & 0 & & & \\
\hline 00005 & & 53509.21 & -52605.7 & & & & & & & & & 17.01 & 16.18 & 15.65 & 0.05 & 0.04 & 0.06 & 0 & & & \\
\hline 00006 & & 53520.13 & -52604.2 & & & & & & & & & 14.57 & 13.01 & 12.27 & 0.01 & 0.01 & 0.01 & 0 & & & 0535201-052604(4) \\
\hline 00007 & & 53504.48 & -52604.1 & 12.14 & 11.25 & 10.89 & 10.15 & 0.03 & 0.04 & 0.03 & 0.03 & & & & & & & 0 & & & 0535044-052604(4) \\
\hline 00008 & & 53505.18 & -52603.4 & 13.54 & 12.88 & 12.52 & 12.04 & 0.01 & 0.01 & 0.03 & 0.12 & & & & & & & 0 & 262 & & \\
\hline 00009 & & 53511.48 & -52602.6 & 9.91 & 9.06 & 8.84 & 8.47 & 0.05 & 0.01 & 0.02 & 0.02 & & & & & & & 0 & 365 & & \\
\hline 00010 & & 53522.57 & -52602.1 & & & & & & & & & 17.18 & 16.56 & 16.37 & 0.09 & 0.08 & 0.08 & 0 & & & \\
\hline 00011 & & 53506.92 & -52600.5 & 13.48 & 13.14 & 12.54 & 11.43 & 0.02 & 0.05 & 0.02 & 0.06 & 13.39 & 12.56 & 12.19 & 0.01 & 0.01 & 0.01 & 0 & 299 & & \\
\hline 00012 & & 53524.34 & -52600.3 & $\begin{array}{l}13.09 \\
13.09\end{array}$ & 12.42 & 12.12 & 11.38 & 0.08 & 0.03 & 0.02 & 0.10 & 13.05 & 12.37 & 12.02 & 0.01 & 0.01 & 0.01 & 0 & 3101 & & \\
\hline 00013 & & 53510.48 & -52600.3 & 13.01 & 12.25 & 11.82 & 11.11 & 0.03 & 0.03 & 0.02 & 0.05 & 12.92 & 12.07 & 11.66 & 0.01 & 0.01 & 0.01 & 0 & 3104 & & \\
\hline 00014 & & 53510.76 & -52600.0 & 15.54 & $\begin{array}{l}13.61 \\
13.0\end{array}$ & $\begin{array}{l}11.02 \\
12.60\end{array}$ & 11.72 & 0.04 & 0.08 & 0.02 & 0.09 & 15.42 & 13.60 & 12.57 & 0.01 & 0.01 & $\begin{array}{l}0.01 \\
0.01\end{array}$ & 0 & & & \\
\hline 00015 & & 53515.42 & -52559.5 & 13.72 & 12.84 & 12.38 & 11.56 & 0.01 & 0.02 & 0.02 & 0.06 & 13.68 & 12.78 & 12.26 & 0.01 & 0.01 & 0.02 & 0 & 3103 & & \\
\hline
\end{tabular}

Note. - The complete version of this table is in the electronic edition of the Journal. The printed edition contains only a sample.

Note. - This table is available only on-line as a machine-readable table. 
Fig. 1.- Color composite near-infrared $\left(\mathrm{JHK}_{\mathrm{S}}\right)$ NTT image of the Trapezium Cluster. Taken with SOFI at the ESO NTT telescope, La Silla, Chile, March 2000. North is up and east is left and the field of view is $5^{\prime} \times 5^{\prime}$. Note the very red objects which trace the location of the parental molecular cloud and lie in an arcing ridge from southwest of the namesake Trapezium stars through the Orion South and the BN/KL regions and then directly north the Trapezium stars. The NTT region is compared to that of other deep near-IR surveys in figure 17.

Fig. 2.- Raw near-infrared infrared luminosity functions. a) Trapezium Cluster $\mathrm{J}$ and $\mathrm{H}$ band Luminosity Functions. The Trapezium HLF is the open histogram and the Trapezium JLF is the shaded histogram. Completeness (90\%) limits are marked by a solid vertical line at $18.15(\mathrm{~J})$ and a broken vertical line at $17.8(\mathrm{H})$. b) Trapezium Cluster K band Luminosity Function. The Trapezium KLF constructed from the FLWO-NTT catalog is compared to the KLF constructed in MLL2000. The $\mathrm{K}=17.590 \%$ completeness limit is demarked by a vertical broken line.

Fig. 3.- Observed control field KLF for the Trapezium Cluster. a) The two histograms are the off-field KLFs obtained as part of the FLWO and NTT observations. The NTT off-fields are aproximately 2 magnitudes deeper than the FLWO off-fields, but the FLWO off-fields covered twice the area of the NTT off-fields. Both are scaled to the size of the Trapezium NTT region. The inset diagram shows the distribution of $\mathrm{H}-\mathrm{K}$ colors for these two off-fields. Their similar narrow widths indicate they are free of interstellar extinction; b) The weighted average of the FLWO and NTT field stars KLFs is compared to the Trapezium Cluster KLF constructed in figure 2(b).

Fig. 4.- Deriving cluster KLF $\mathrm{M}-\mathrm{A}_{V}$ Completeness limits. a) Trapezium Cluster $(\mathrm{H}-\mathrm{K}) / \mathrm{K}$ color-magnitude diagram. Only stars falling within the Trapezium NTT region are shown. Stars selected to fall into our mass-limited, extinction-limited sample are indicated by filled circles. The distribution of sources in this color-magnitude space is compared to the location of the pre-main sequence 0.2 and 0.8 Myrs isochrones from DM97. Reddening vectors representing 17 magnitudes of visual extinction are shown for 2.50, 0.08 and $0.02 \mathrm{M}_{\odot}$ stars at the mean age assumed for this cluster (0.8 Myrs). The zero-age main sequence (Kenyon \& Hartmann 1995; Bessell 1995) is shown for 03-M6.5 stars at a distance of 400pc. b) Effects of mass/extinction limits on the cluster KLF. Comparison of the $\mathrm{M}-\mathrm{A}_{V}$ limited KLF derived from (a) to the raw Trapezium KLF (see figure $2 \mathrm{~b})$. Sensitivity $(\mathrm{K}=18.1)$ and completeness $(\mathrm{K}=17.5)$ limits are shown as vertical broken lines.

Fig. 5.- Testing the contribution of reddened field stars to the Trapezium Cluster KLF. Panels A \& B: The first pair of panels displays the observed off-field KLF (figure 3b ) reddened by "background shields" of extinction in the form of gaussian distributions (of $\mathrm{A}_{V}$ ) centered at 10 (panel A) and 25 (panel B) magnitudes; Panels C \& D: The second two panels are the observed off-field KLF reddened by an extinction map converted from a $\mathrm{C}^{18} \mathrm{O}$ map. The two panels represent the variation 
in the predicted field star contamination as a function of the uncertainty in the $\mathrm{C}^{18} \mathrm{O}$ to $\mathrm{A}_{V}$ conversion; Panels E \& F: The final panels are of the same experiment as performed in $\mathrm{C} \& \mathrm{D}$, but they have been filtered to reflect the actual contribution due to the $\mathrm{M}-\mathrm{A}_{V}$ limits.

Fig. 6.- Infrared color distributions for the FLWO-NTT Trapezium observations. a) Histogram of the observed $(\mathrm{H}-\mathrm{K})$ color for the FLWO-NTT Trapezium sources. The subset of these sources which lack $\mathrm{J}$ band measurements are indicated by the shaded histogram; b) Trapezium Cluster ( $\mathrm{H}$ K) vs ( J - H) color-color diagram for the NTT region. Symbols indicate if the source's colors were taken from the FLWO catalog (filled circles, JHK) or the NTT catalog (open circles, $\mathrm{JHK}_{\mathrm{S}}$ ).

Fig. 7.- Probability distributions of source reddening for the Trapezium Clusters sources. a) Extinction Probability Distribution Function (EPDF) for the Trapezium Cluster sources. Plotted are three variations in the EPDF under different assumptions of the typical $(\mathrm{H}-\mathrm{K})_{\text {(star-disk) }}$ color for the $20 \%$ of the stars lacking $\mathrm{J}$ band measurements. See section 3.1 .1 for derivation. It is compared to the extinction probability distribution function integrated from the $\mathrm{C}^{18} \mathrm{O} \rightarrow \mathrm{A}_{V}$ map. Note that they are not well separated distributions. A broken vertical line indicates the $\mathrm{A}_{V}=17$, $\mathrm{M}-\mathrm{A}_{V}$ limit; b) Infrared Excess Probability Distribution Function (IXPDF) for the Trapezium Cluster. The derived $\mathrm{H}-\mathrm{K}_{\mathrm{S}}$ color excess distribution function is assumed to reflect a magnitude excess at $\mathrm{K}$ band alone. See section 3.1.2 for derivation.

Fig. 8. - Effect of the Trapezium Cluster EPDF on cluster luminosity functions. A model luminosity function of the Trapezium (using the Trapezium IMF derived in MLL2000) is convolved with the Trapezium Cluster EPDF at four different wavelengths. Reddening effects are most significant at $\mathrm{I}$ and $\mathrm{J}$ bands and are minimized at $\mathrm{K}$ band.

Fig. 9.- Best fitting model KLFs and corresponding cluster IMFs. Top panels show the $\mathrm{M}-\mathrm{A}_{V}$ limited, background subtracted Trapezium KLF (histogram) and best fit reddened model KLFs (unconnected filled circles). Bottom panels display the resulting underlying IMFs and corresponding chi-sq probabilities. Panel (a) shows models fit between $K=7.5$ and 14.5, the same range fit in MLL2000. Panel (b) shows three power-law IMF fits to the secondary peak in the cluster $\mathrm{KLF}$ at $\mathrm{K}=15.5$, which correspond to low $\chi^{2}$ probability due to the presence of structure and the secondary KLF peak.

Fig. 10.- Contours of $\chi^{2}$ probability for the 5 parameters of the underlying three power-law IMF. Two parameters are compared in each panel while fixing the other three to a best fit value. Solid contours are best fit ranges from models that include source reddening. Dashed contours are from best fit models without source reddening. Contour levels are shown at intervals 95, 90, 70, 50 and $30 \%$ confidence. Panels (a) to (c) are shown for fits to $K=14.5$ and panel (d) is shown for fits to $\mathrm{K}=15$. 
Fig. 11.- Overall Trapezium IMF. a) Trapezium $\mathrm{M}-\mathrm{A}_{V}$ limited KLF fit over the entire luminosity range by employing a four power-law IMF that truncates at the lower mass limit our standard merged PMS tracks $\left(\sim 17 \mathrm{M}_{\text {Jup }}\right)$. The brown dwarf IMF breaks from a steady decline $\left(\Gamma_{3}=0.73\right)$ between 0.03 and $0.02 \mathrm{M}_{\odot}$ and rises to the mass limit of the PMS tracks. The rapid tailing off of the cluster KLF does imply that the sub-stellar Trapezium IMF is characterized by a truncation near $17 \mathrm{M}_{\text {Jup }}$ (see section 4.1.2). Above the $m_{3}$ mass break the IMF is that described in table 1 for fits to $\mathrm{K}=15$. b) Best fit Trapezium IMF and range of Trapezium IMFs derived from KLF modeling. Hatched areas are derived from the range of $90 \%$ confidence contours for KLF fits. Solid line is the best fit Trapezium IMF listed in eq. 1. The Trapezium IMF is also compared to the log-normal Miller \& Scalo (1979) field star IMF.

Fig. 12.- The Trapezium Sub-Stellar IMF. a) Best fit Model KLFs based on multi-power law IMFs are compared to the $\mathrm{M}-\mathrm{A}_{V}$ Trapezium KLF. The Trapezium Sub-Stellar $\mathrm{M}-\mathrm{A}_{V} \mathrm{KLF}$ is shown with (solid histogram) and without (dashed histogram) correction for background field stars. Error bars are from counting statistics and the vertical dotted line demarks the $\mathrm{K}=17.5$ completeness limit. Model KLFs best fit to the $\mathrm{M}-\mathrm{A}_{V} \mathrm{KLF}$ with (filled circles) and without (open circles) background correction are also shown, having error bars that corresponding to the $1 \sigma$ variation of that KLF bin for 50 iterations of the model. b) Derived Sub-Stellar Trapezium IMF using the B97 PMS tracks. Similar to the sub-stellar IMF derived using our standard PMS models (figure 11b), the derived Trapezium brown dwarf IMF steadily declines, breaks and forms a significant secondary peak at $0.014-0.013 \mathrm{M}_{\odot}$ before sharply declining below the deuterium burning limit. Our sample completeness limit $\left(0.01 \mathrm{M}_{\odot}\right)$ is displayed as a vertical dotted line. The IMF fit range allowed by the KLF modeling is graphically displayed and are best fit 3 power-law IMFs from fits with and without background correction are shown (see table 2).

Fig. 13.- A Monte Carlo simulation of the Trapezium Sub-Stellar IMF. Fifty (50) samples of 150 brown dwarfs were drawn from the best fit Trapezium Sub-Stellar IMF derived from B97 tracks, and each was binned into histogram form using equal sized log mass bins. The plotted histogram represents the average of that bin for the iterations, and the error bars represent the derived $1 \sigma$ standard deviation of that IMF bin from the Monte Carlo simulation.

Fig. 14. - Comparison of theoretical mass-luminosity (infrared K magnitude) relations. Theoretical pre-main sequence mass-luminosity relations were extracted from six different sets of evolutionary models (see table 3). In all cases the intrinsic model quantities (luminosity, effective temperature) were converted to $\mathrm{K}$ magnitudes using a single $\mathrm{T}_{\text {eff }}$-bolometric correction relationship. Shown for 6 sets of cluster mean ages: 0.1 (a); 0.5 (b); 1 (c); 2 (d); 3 (e), and 5 (f) Myrs. Baraffe et al., (1998) models do not include models for ages less than 1 Myrs.

Fig. 15.- Comparison of theoretical mass- $\mathrm{T}_{\text {eff }} /$ spectral type relations (figure adopted from 
D'Antona 1998). The spectral type - effective temperature - mass relationships were taken directly from the 6 sets of PMS models at 1 (a) and 5 (b) Myrs. Also shown is the gravity (dwarf vs sub-giant) dependence of the spectral type to effective temperature calibration for late type PMS stars. Because very young sub-solar mass stars and brown dwarfs are primarily on vertical Hayashi contraction tracks in the HR diagram, there is theoretically a close correspondence between effective temperature and mass. The effective temperature to spectral type scale (right hand y-axis) is a cool dwarf scale ( summed from Kenyon \& Hartmann 1995; Bessell 1995; Wilking et al. 1999). The inset spectral sequence is the hotter sub-giant temperature-spectral scale tuned by Luhman (1999).

Fig. 16.-Comparison of five published Trapezium IMFs derived from IR photometry. All derivations used the DM97 PMS tracks for masses less than $1 \mathrm{M}_{\odot}$. Table 4 summarizes differences among the derivation methods. The HC2000 Trapezium IMF corresponds to their $\mathrm{A}_{V}<10 \mathrm{lim}-$ ited sample. The MLL2000 IMF is fit (g) from their table 2.

Fig. 17.- Comparison of the survey areas of recent deep infrared studies of the Trapezium Cluster. The two shaded regions represent the 6.5 × 6.5 FLWO survey and the $5^{\prime} \times 5^{\prime}$ NTT survey presented in this work. Also shown are the NICMOS survey (Luhman et al., 2000, solid black border), the Keck survey (Hillenbrand \& Carpenter 2000, solid white border), and the UKIRT survey (Lucas $\&$ Roche 2000, broken black border). The locations of luminous cluster members, spectral types B3 and earlier, are shown as white stars. 
Figure 1 
Figure 2a

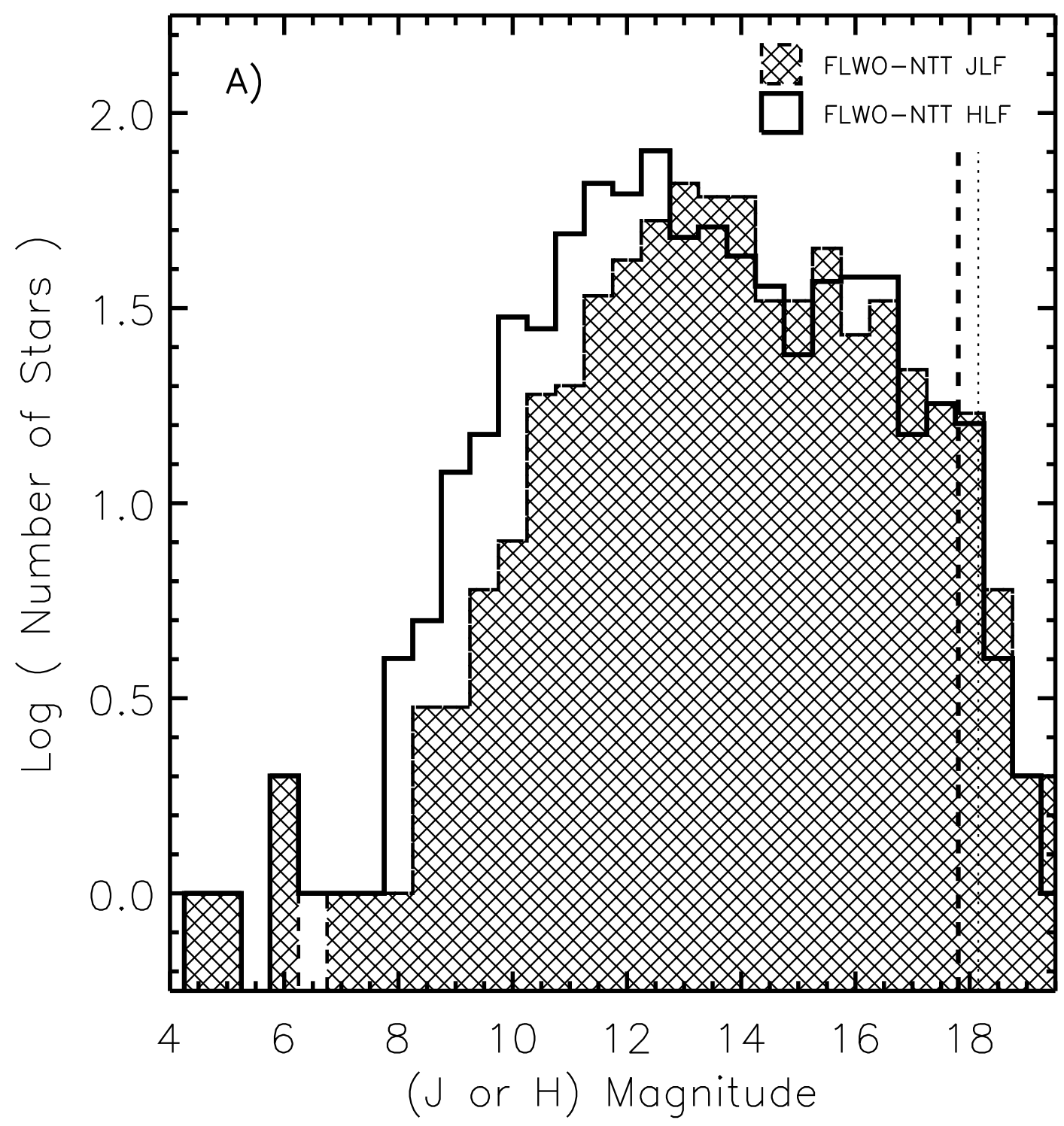


Figure $2 b$

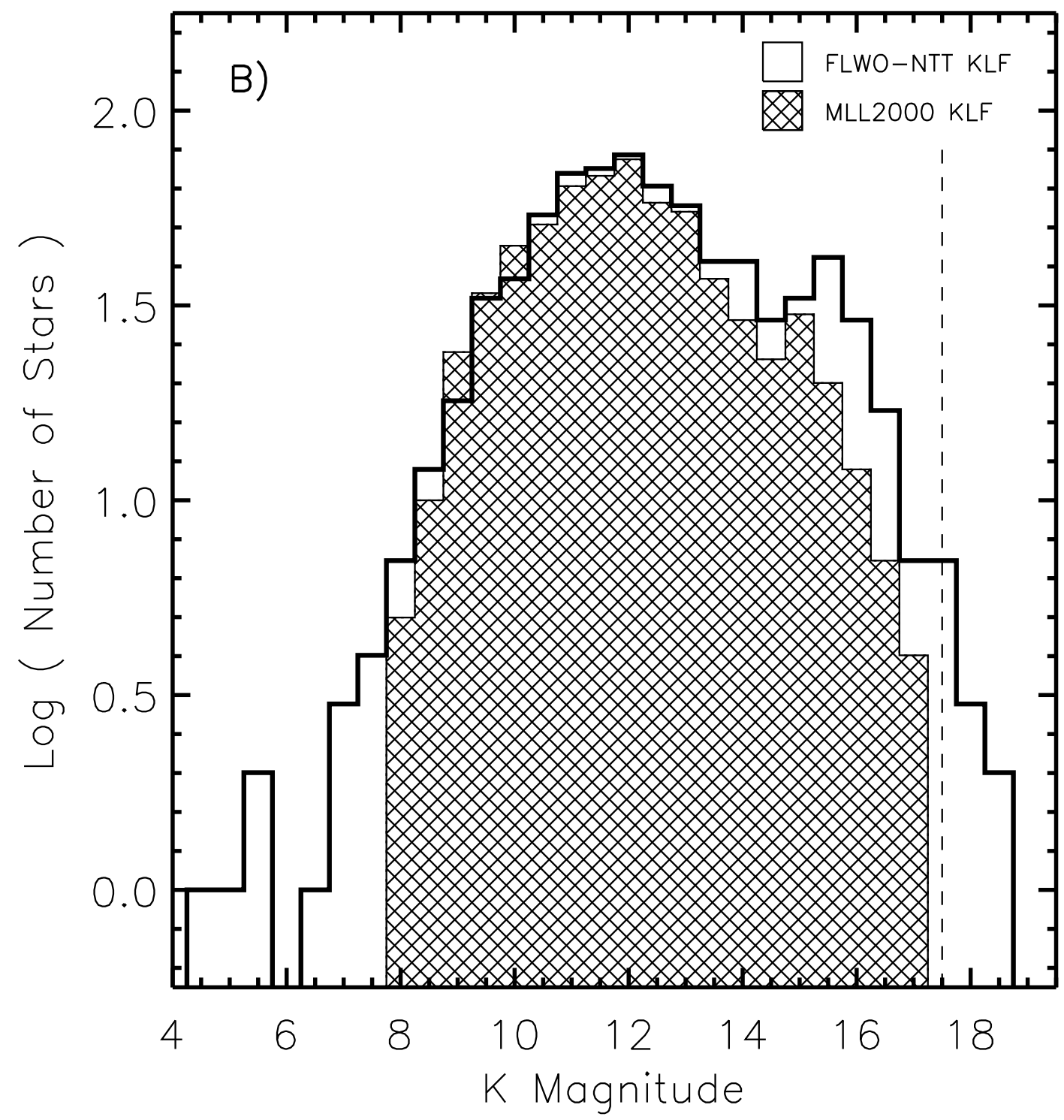




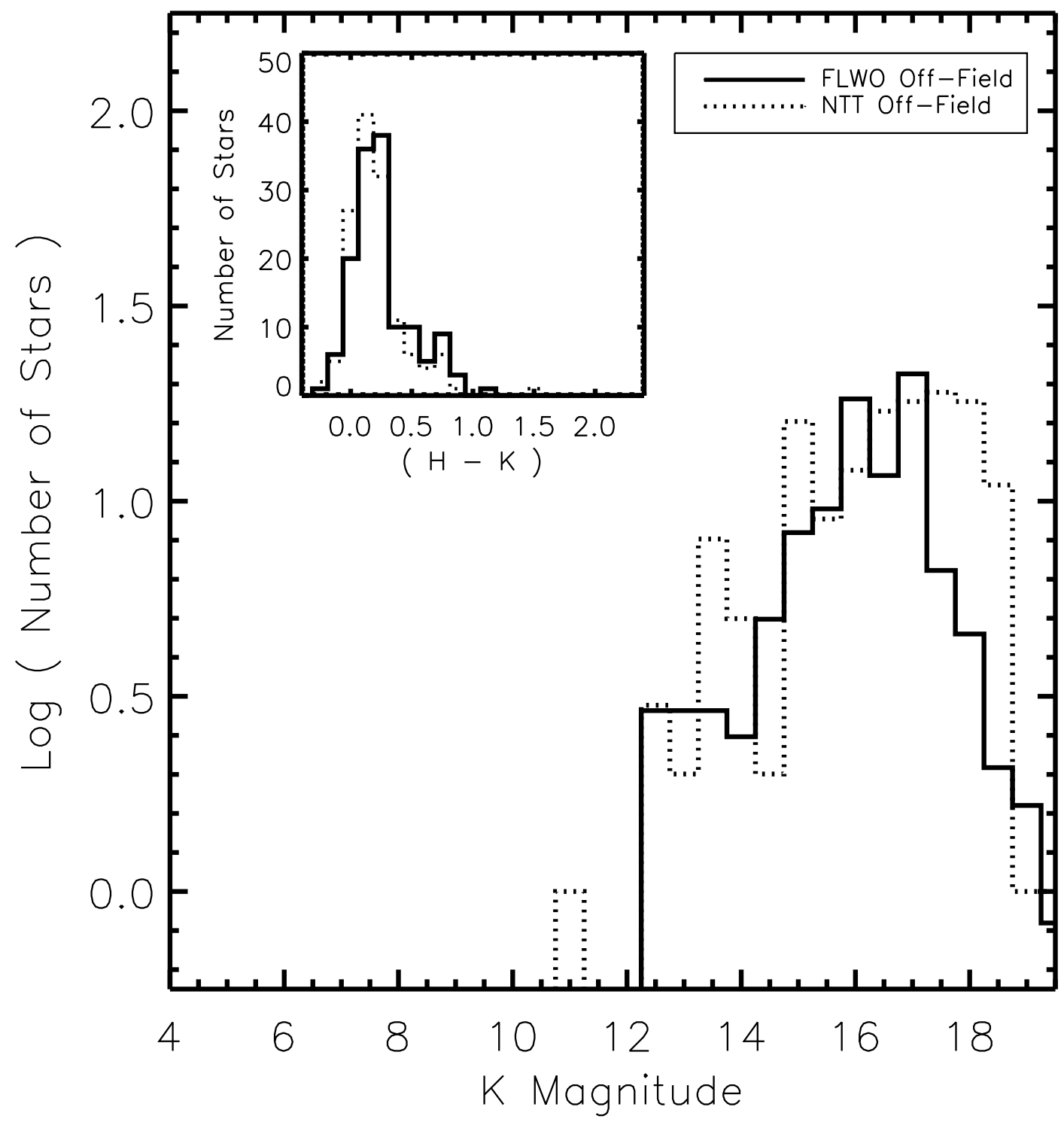


Figure $3 b$

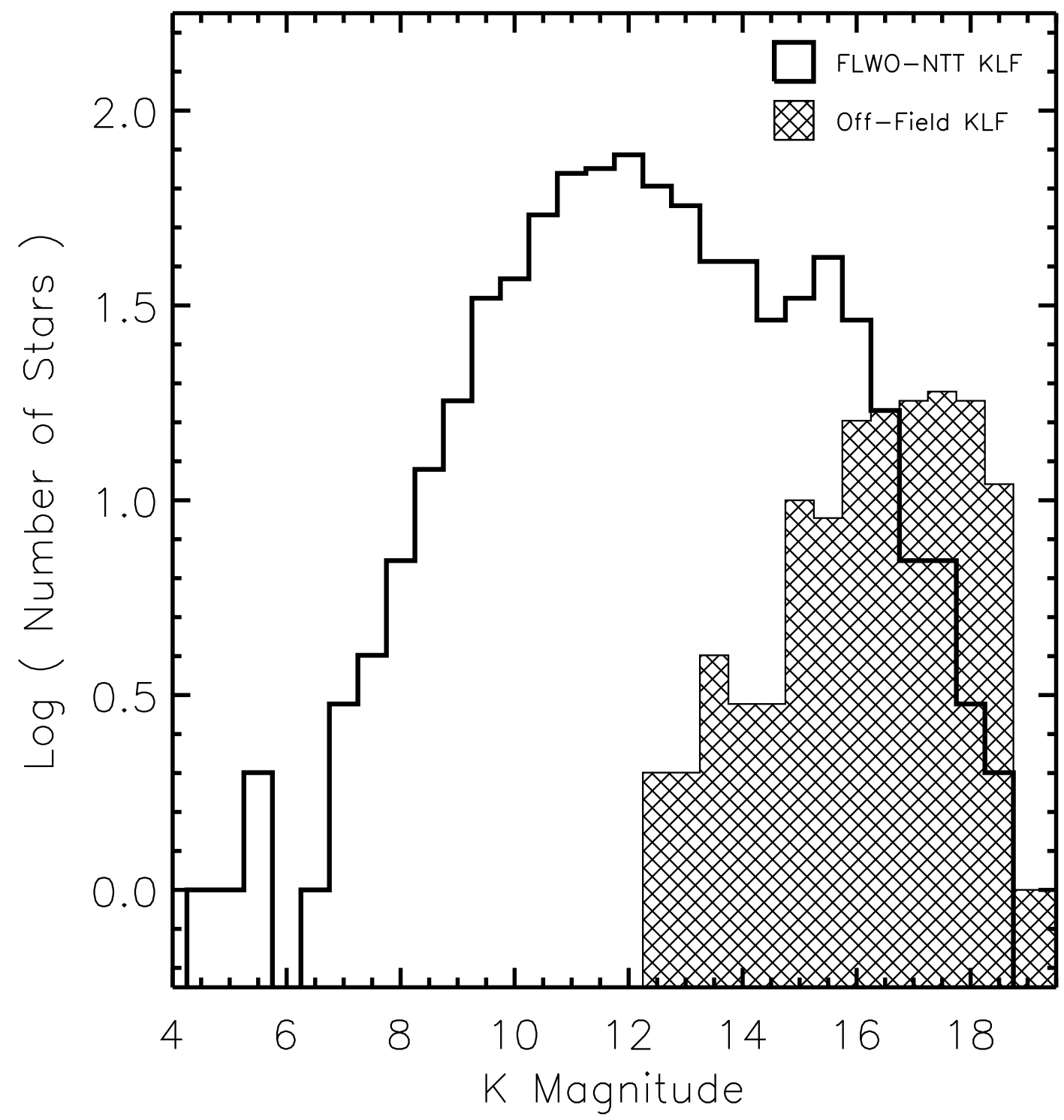




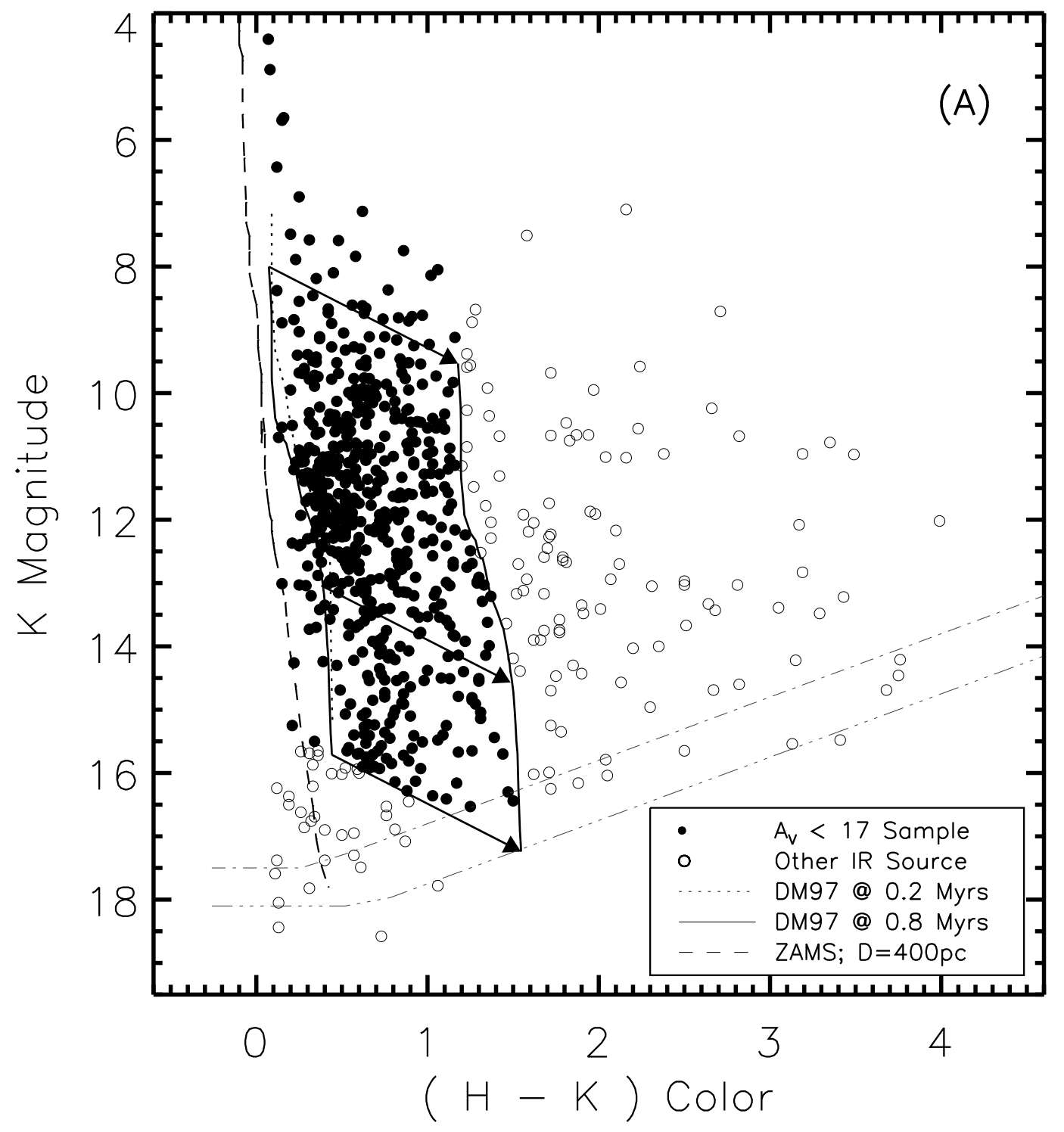


Figure $4 b$

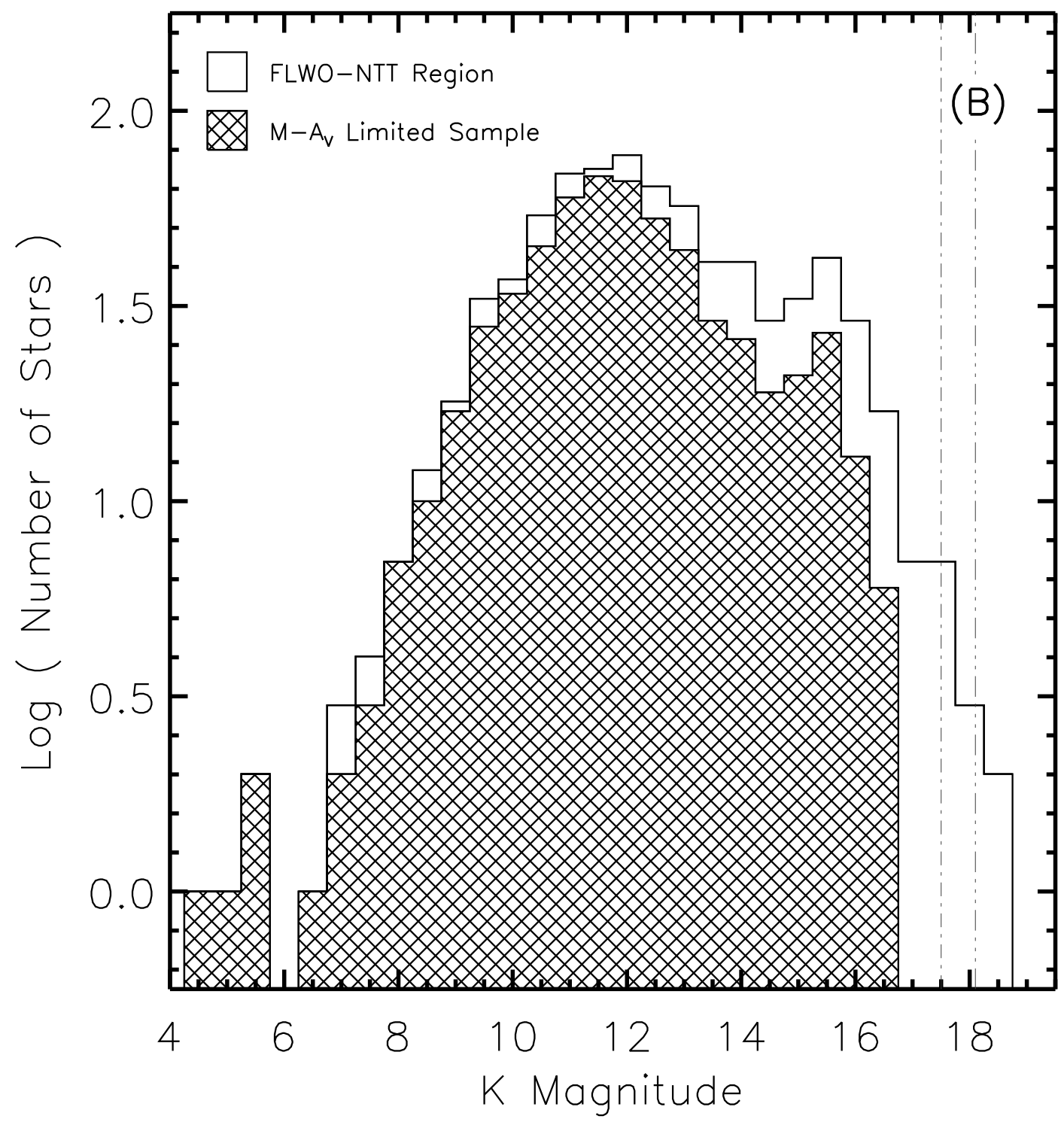



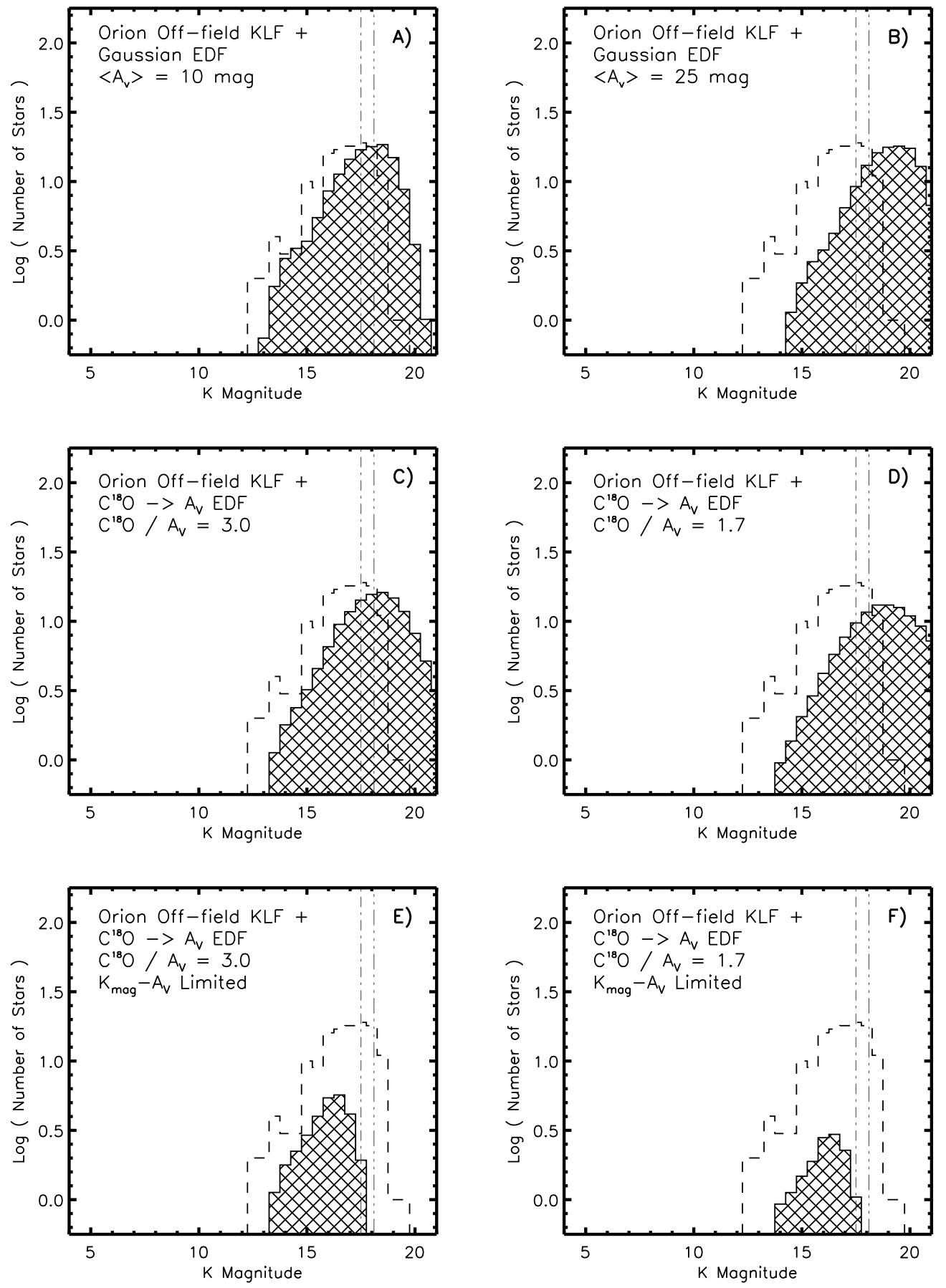


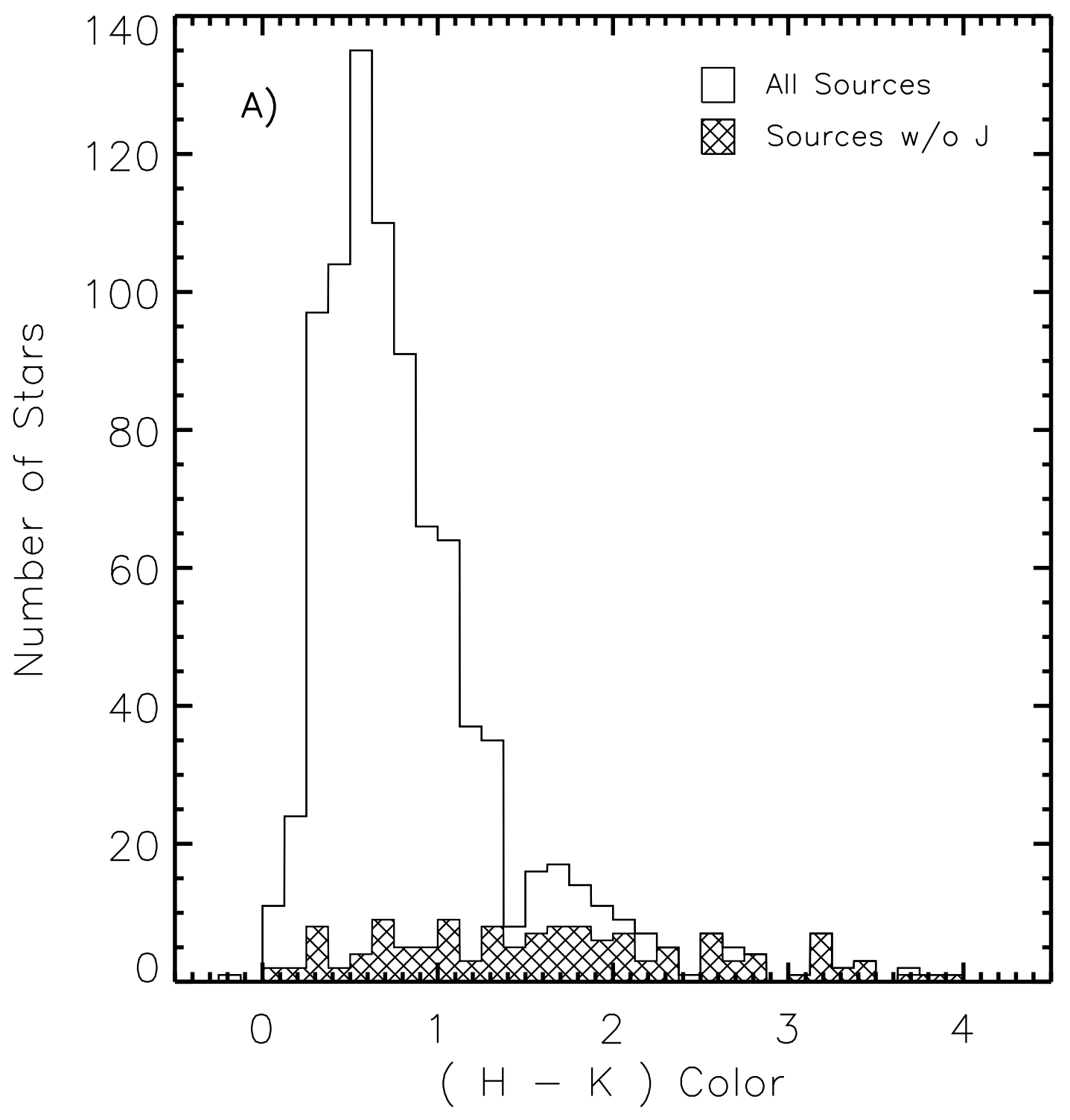




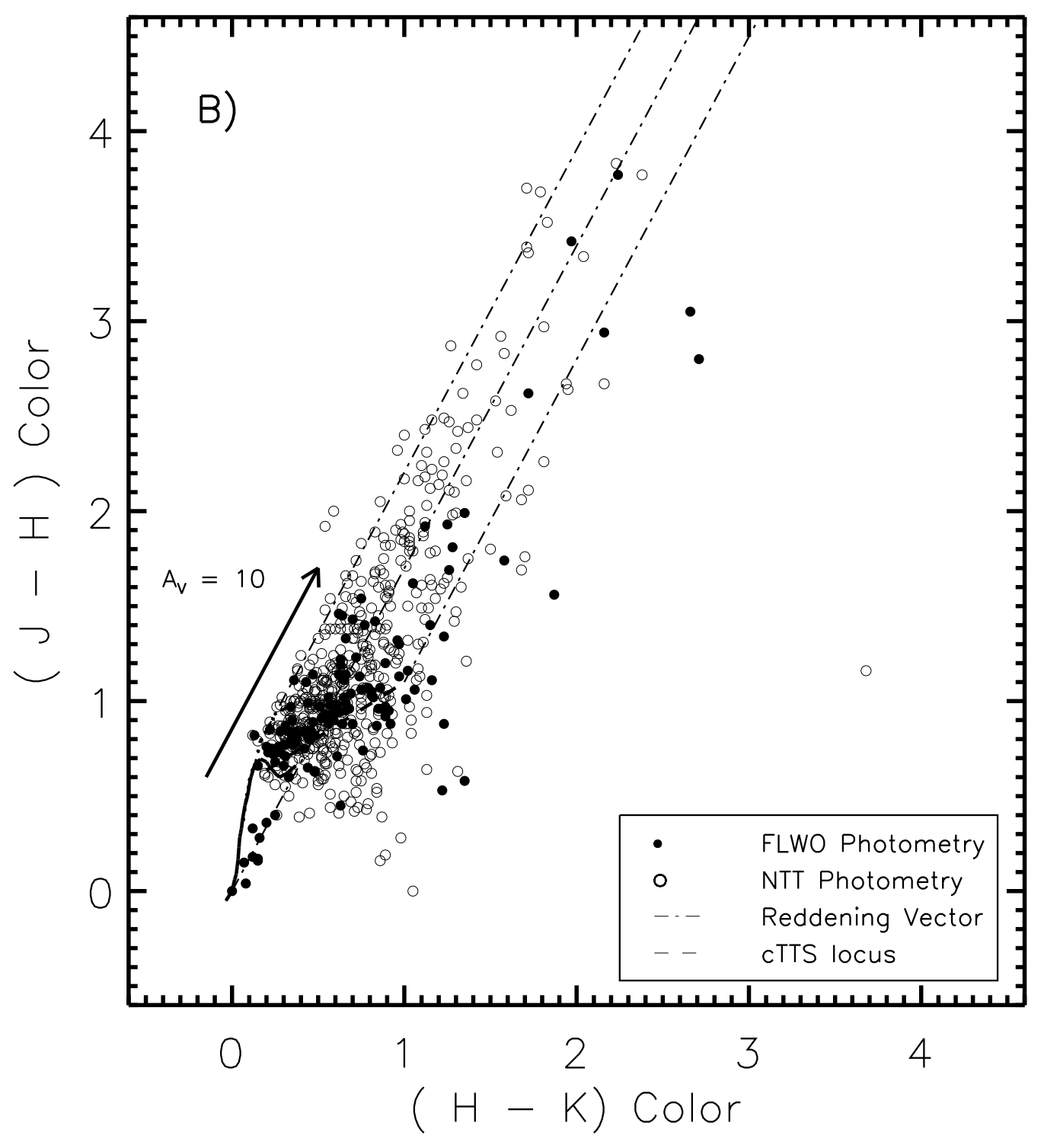




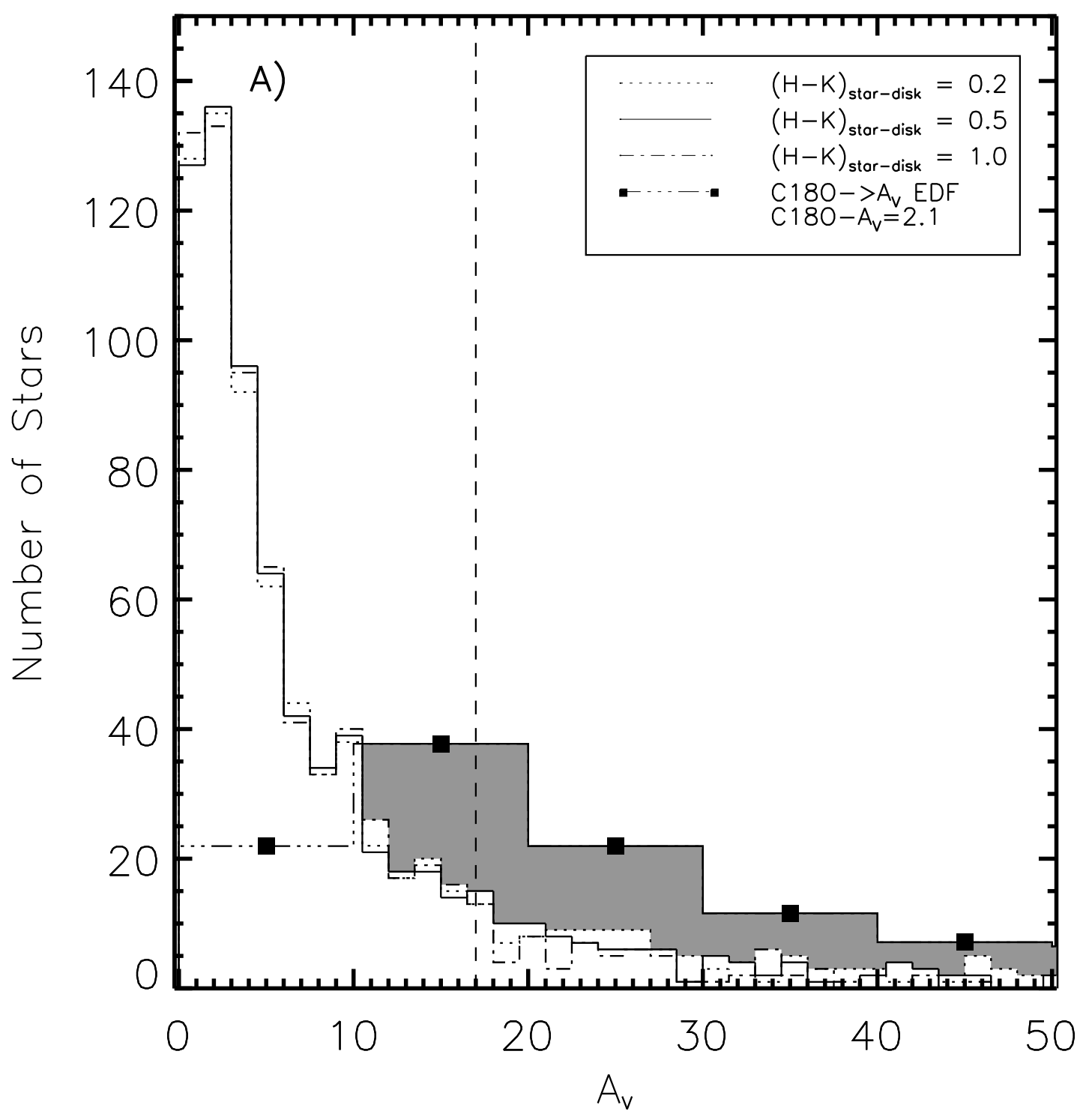


Figure $7 b$

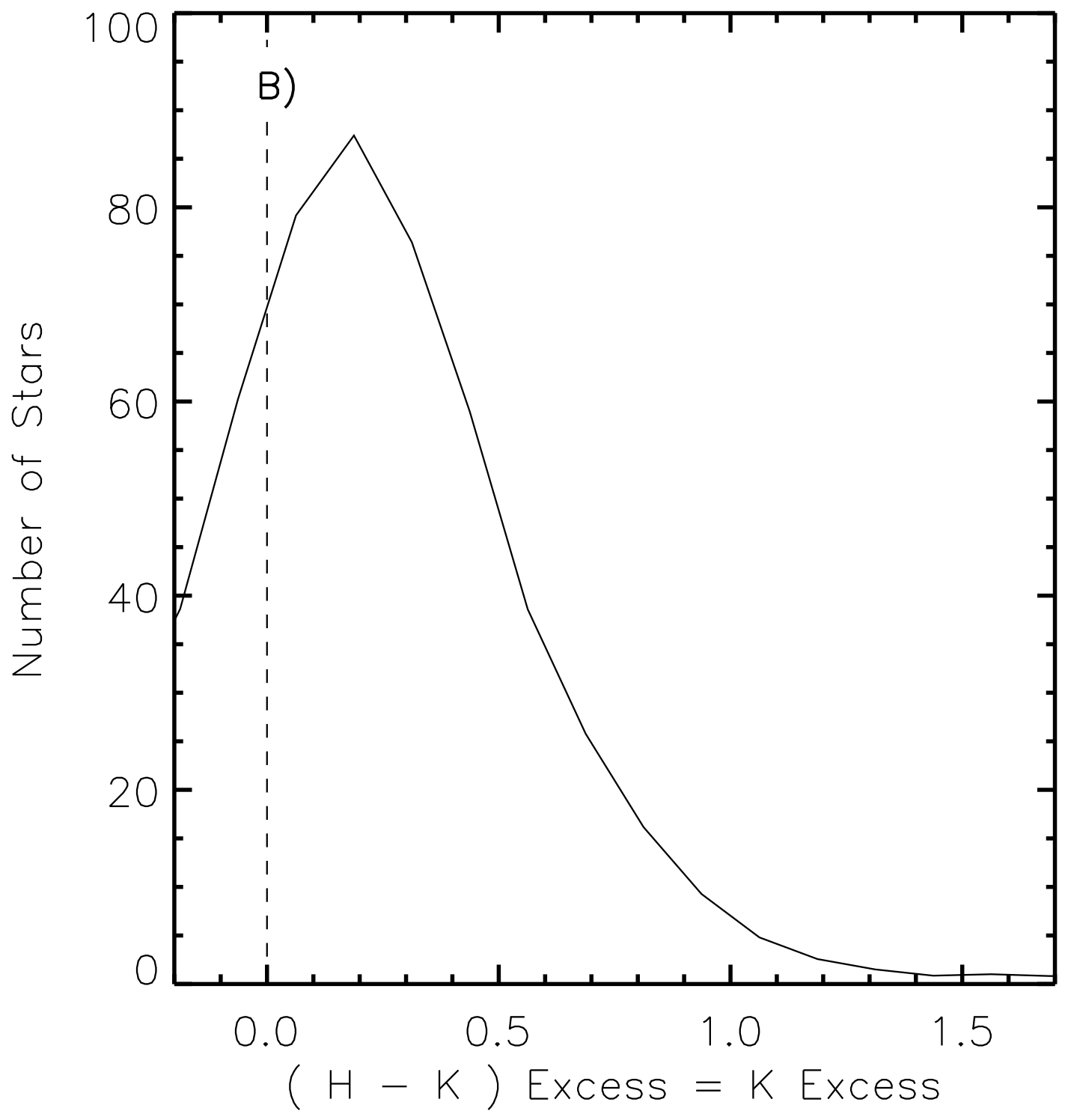



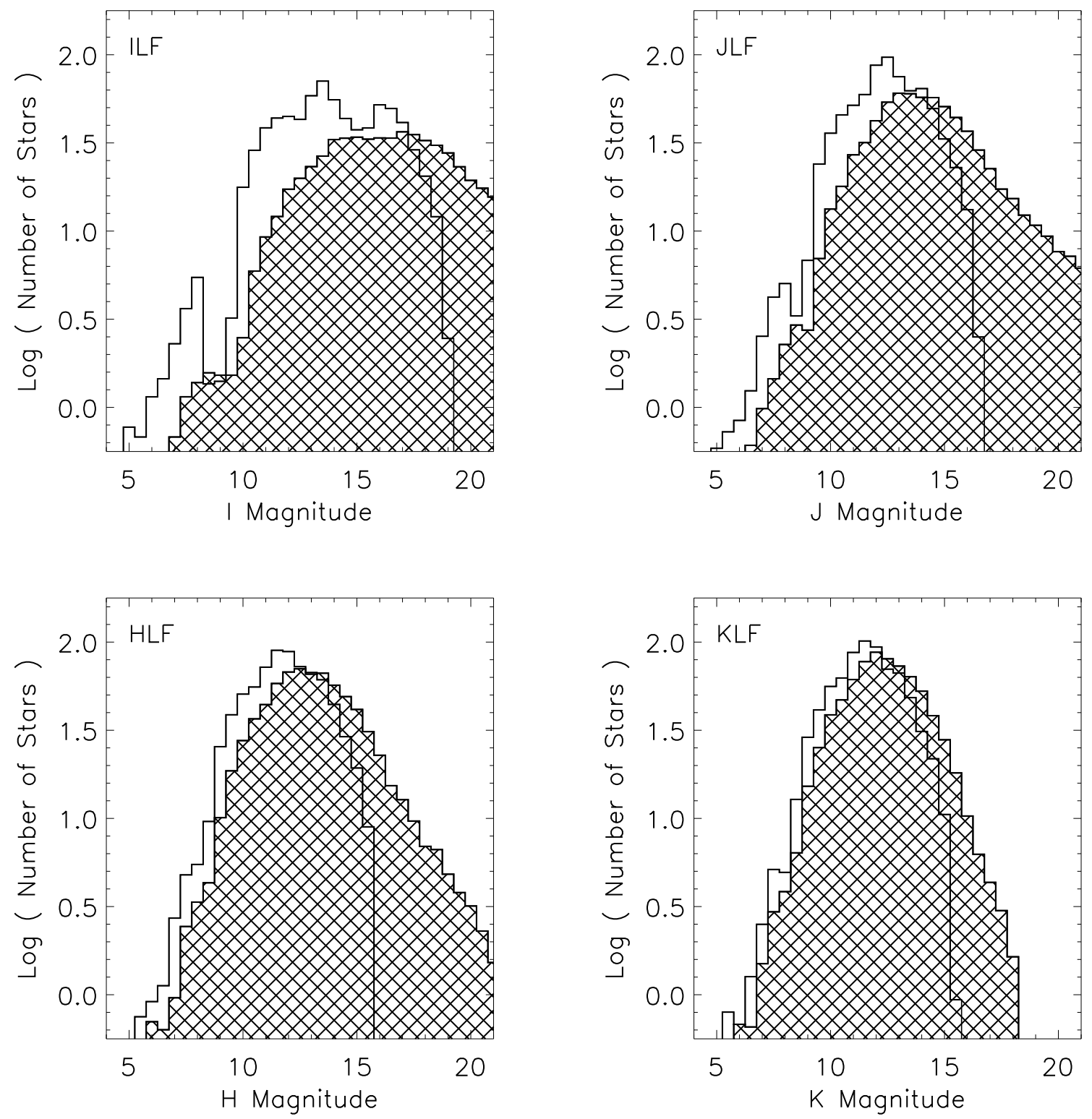
Figure 9
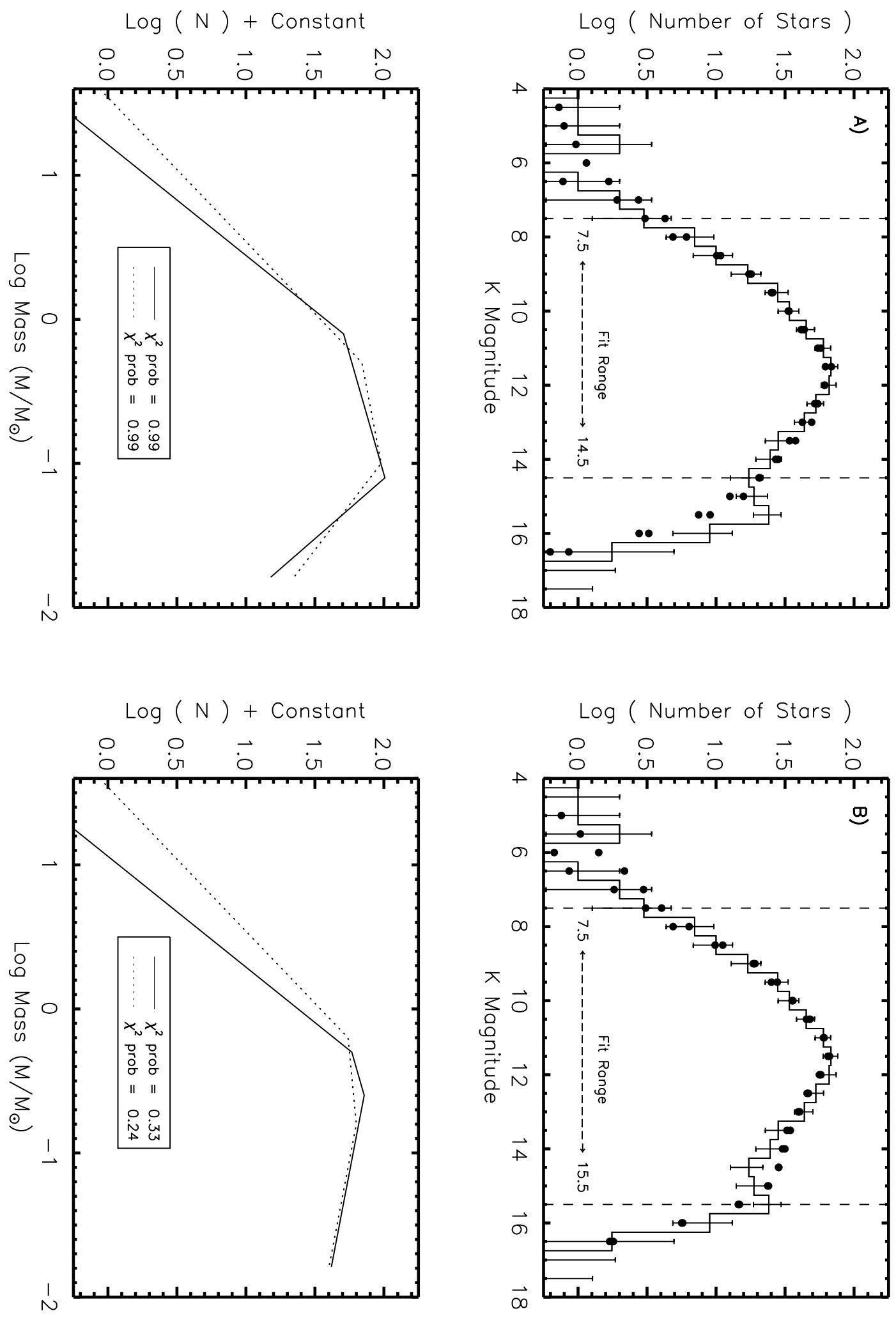

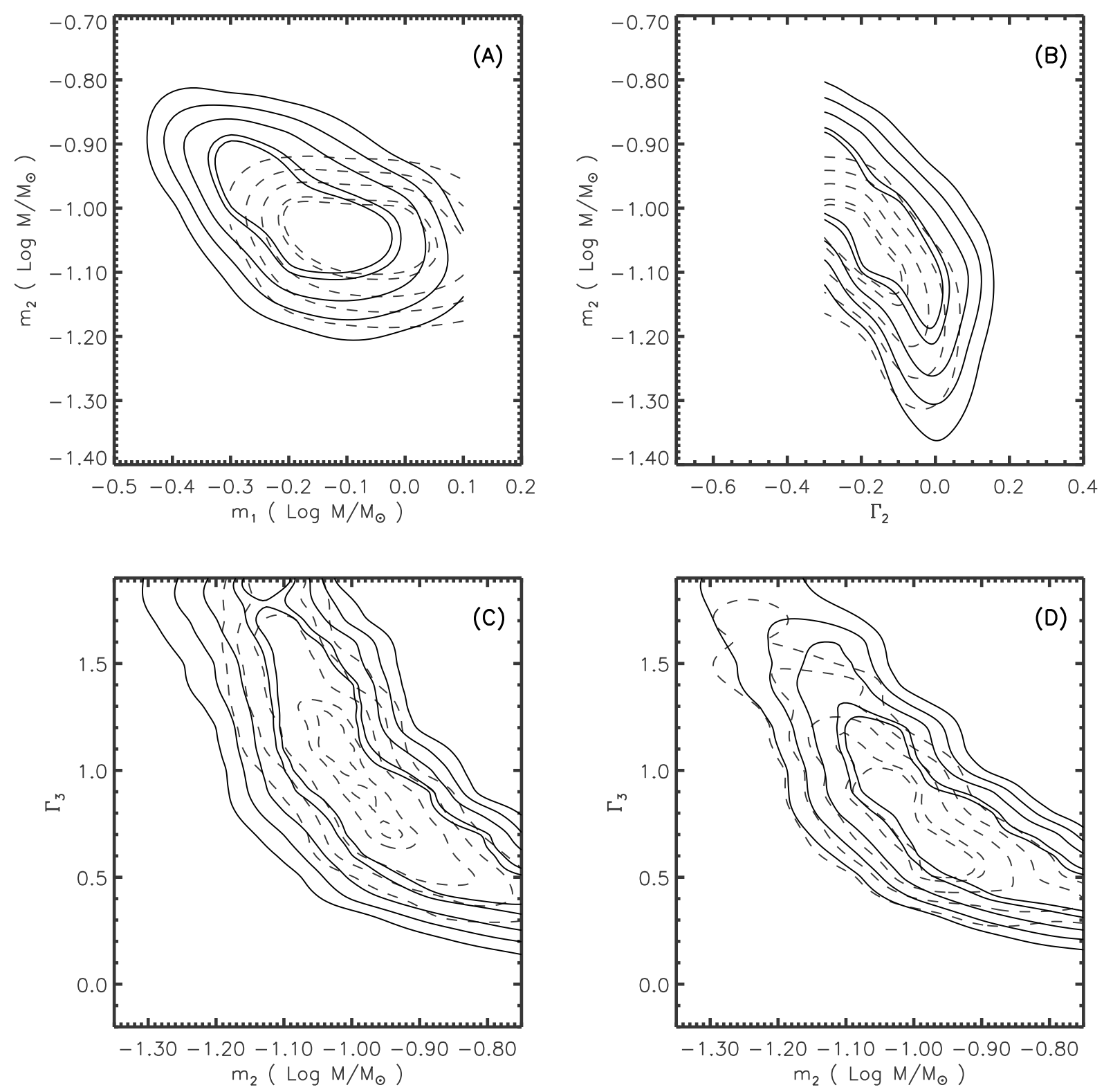
Figure 11a

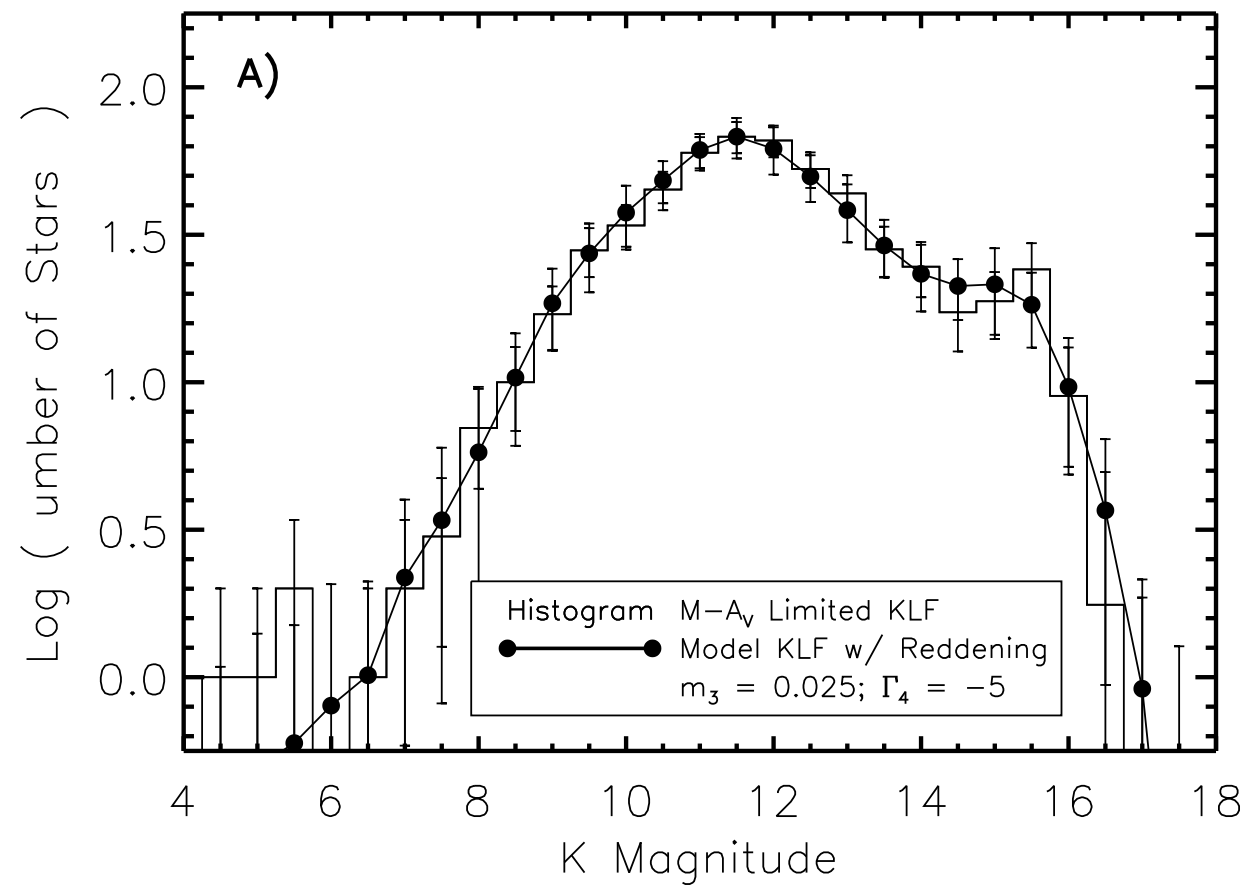


Figure $11 b$

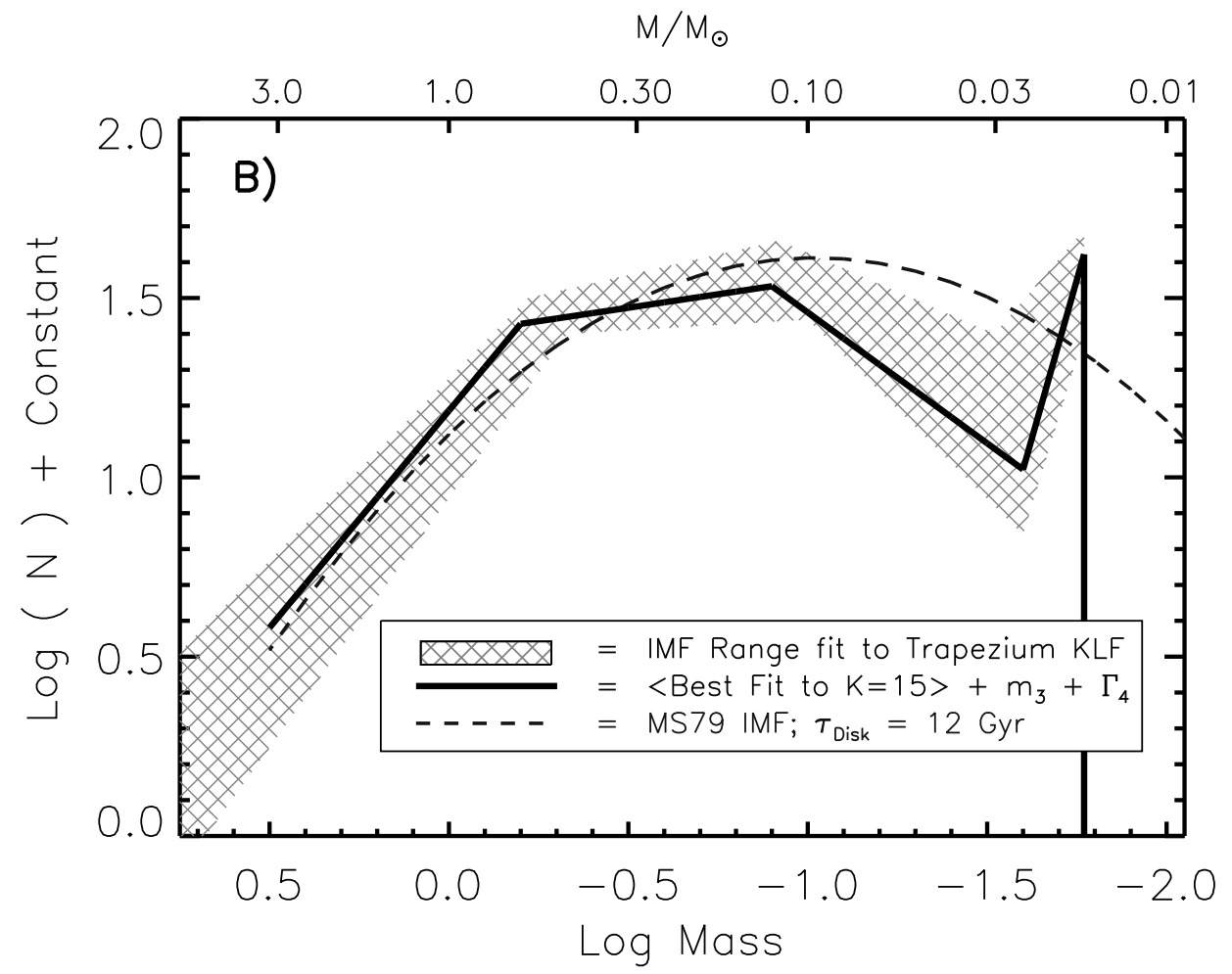


Figure 12a

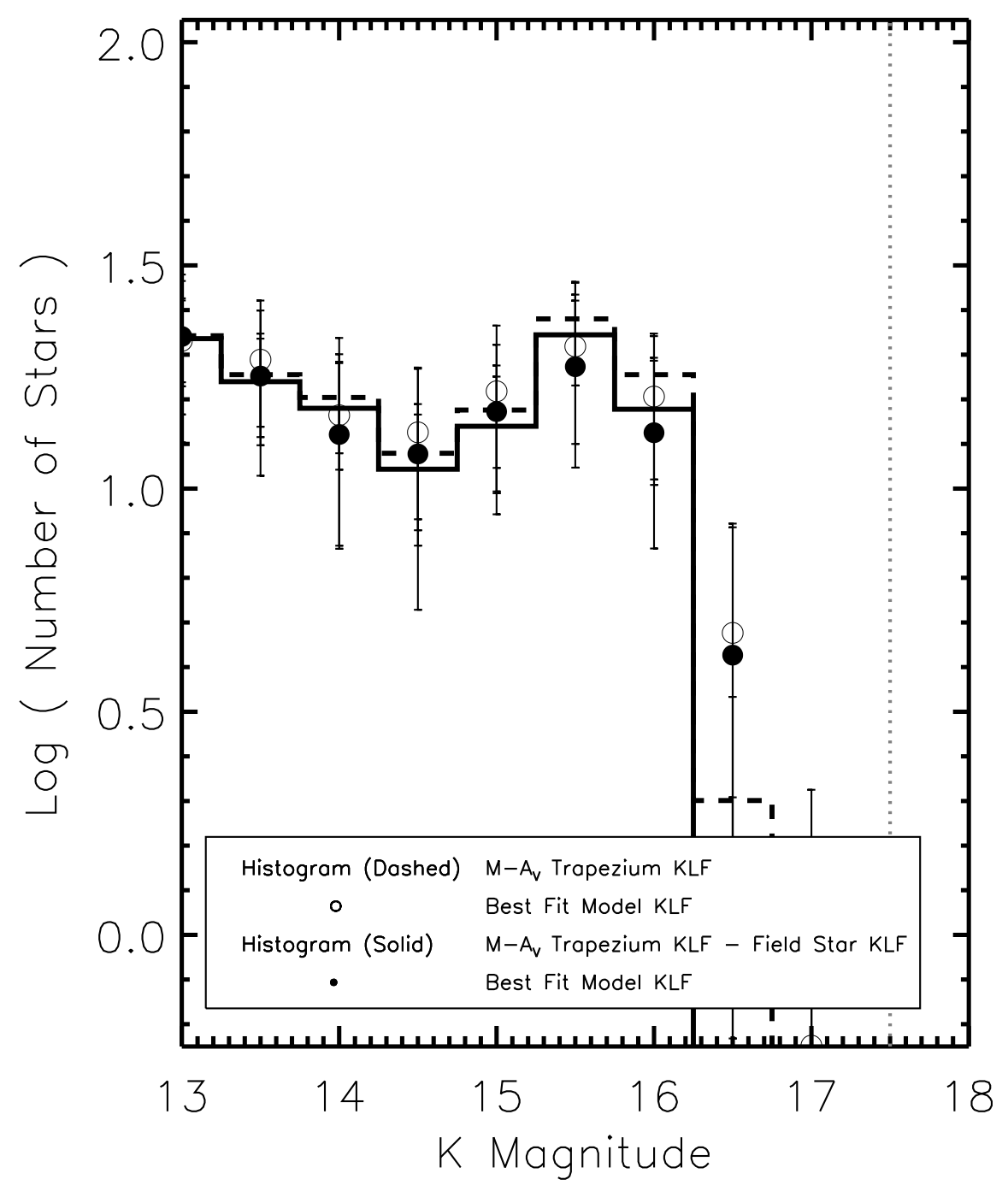


Figure $12 b$

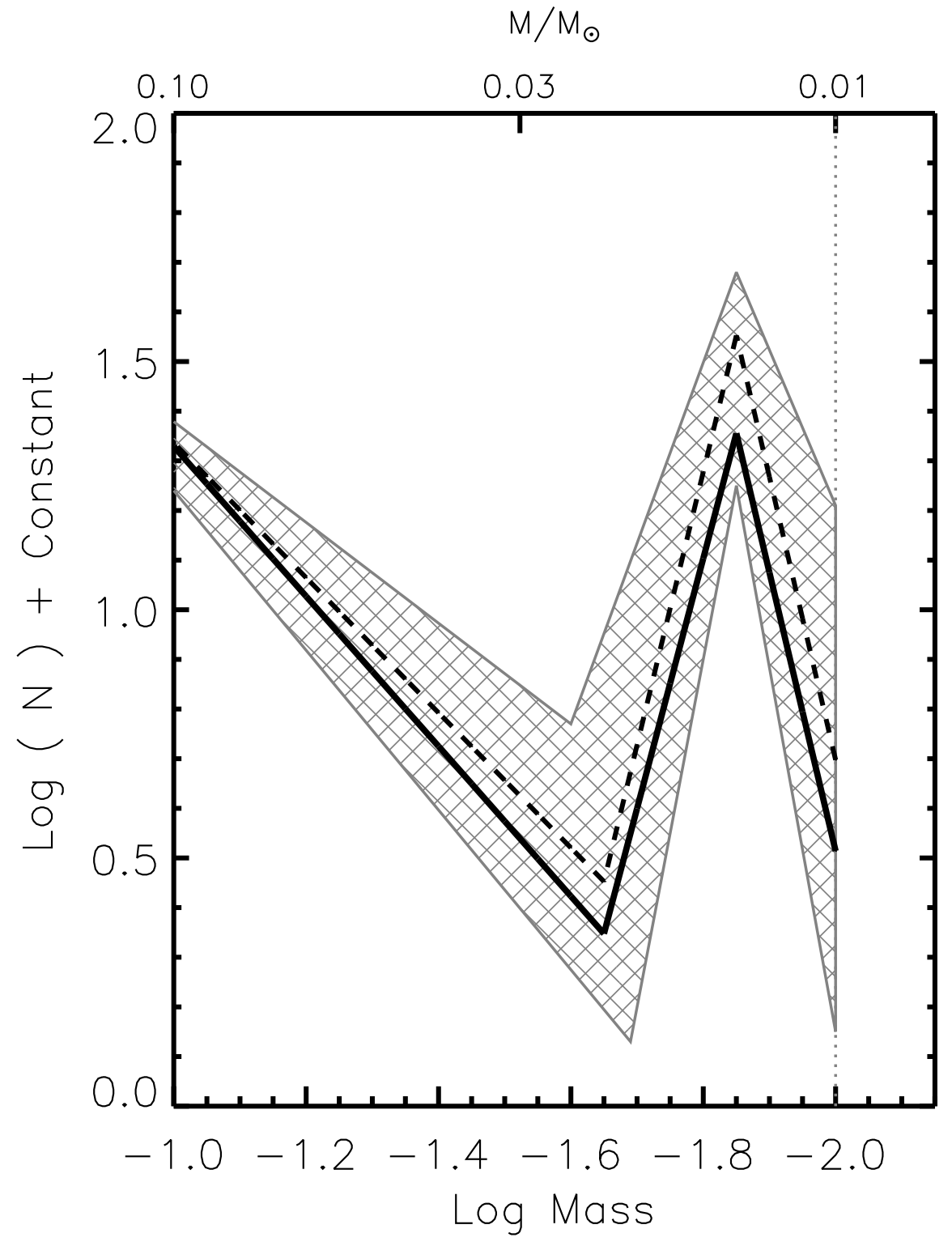


Figure 13

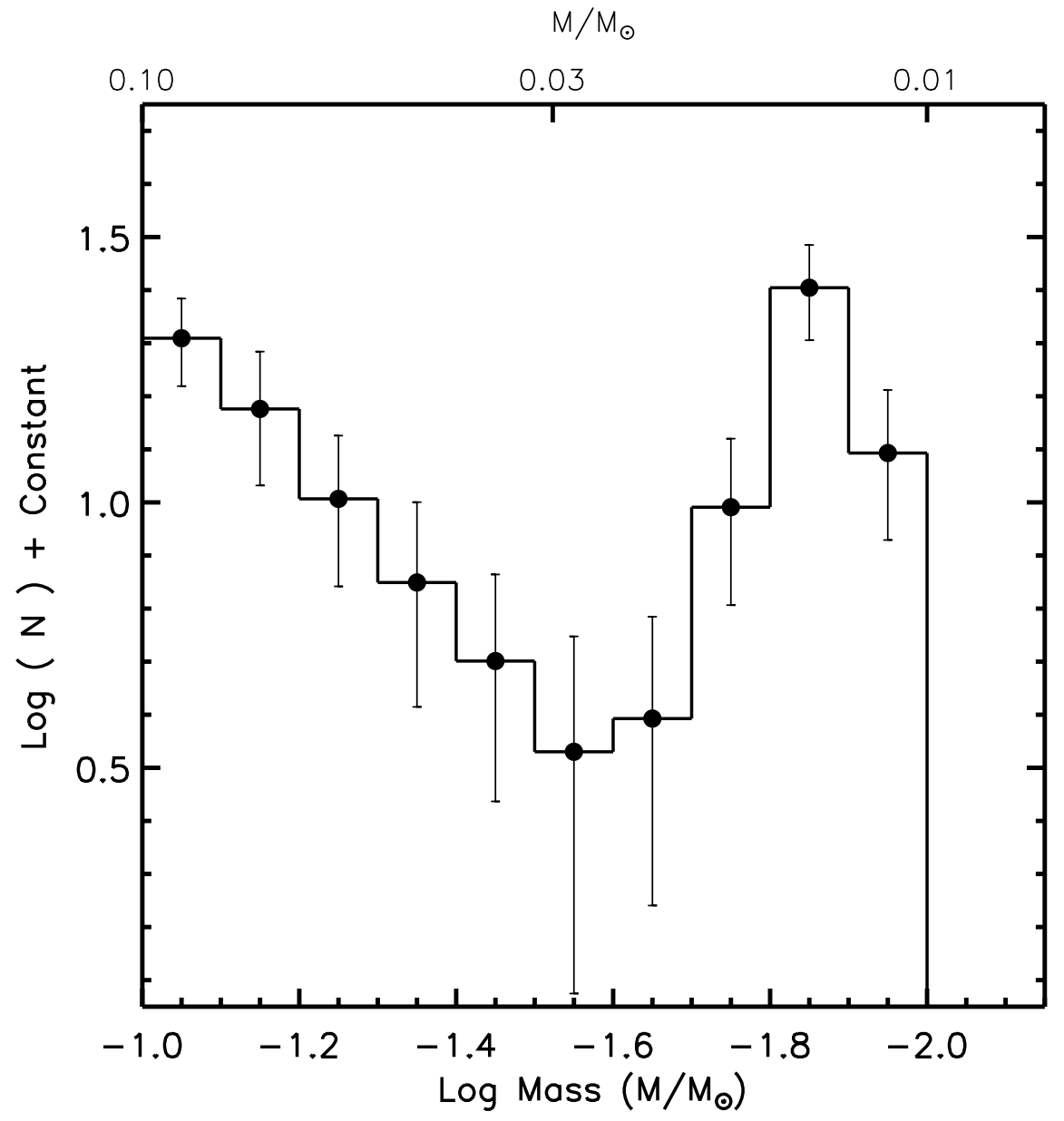




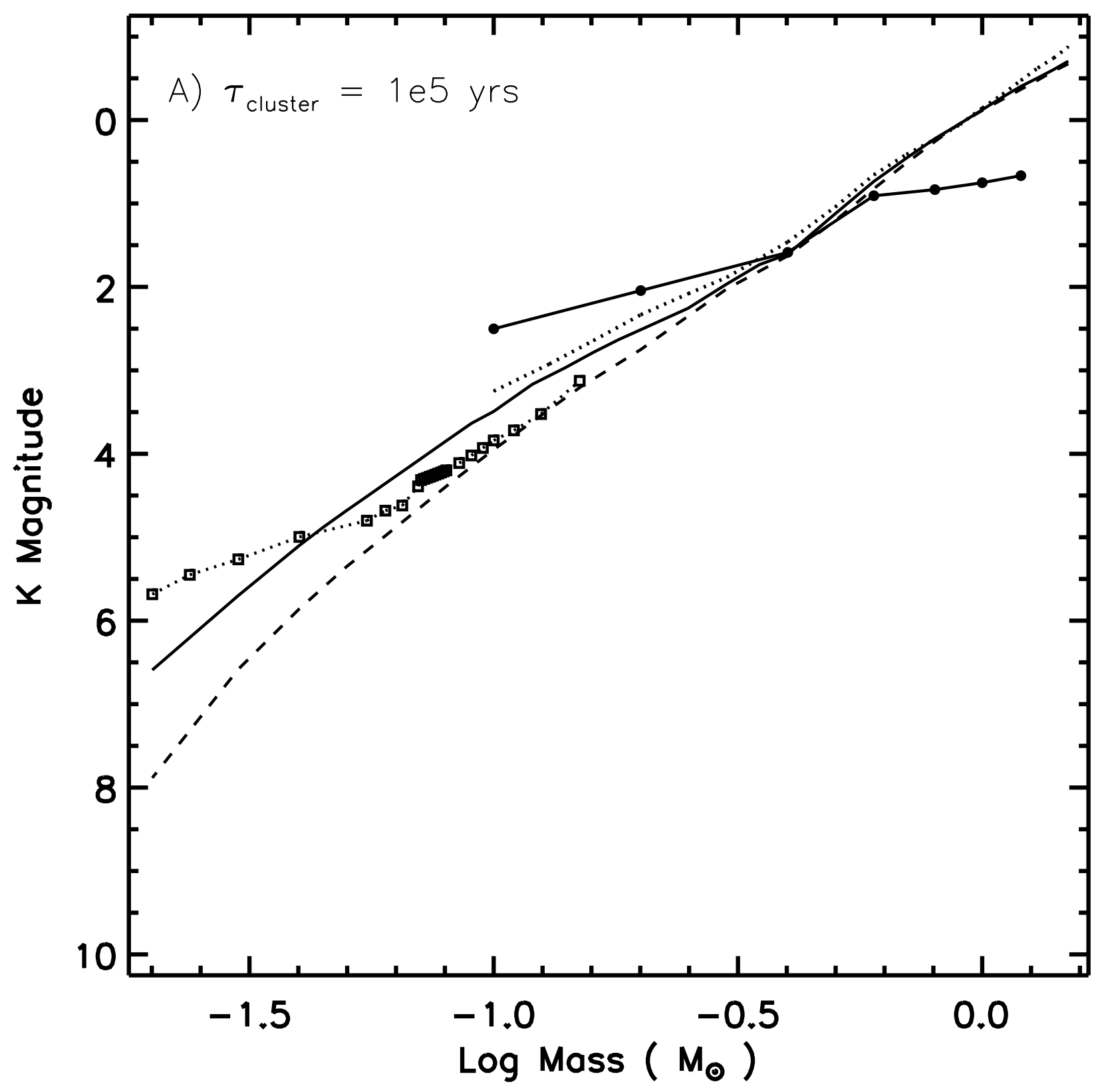




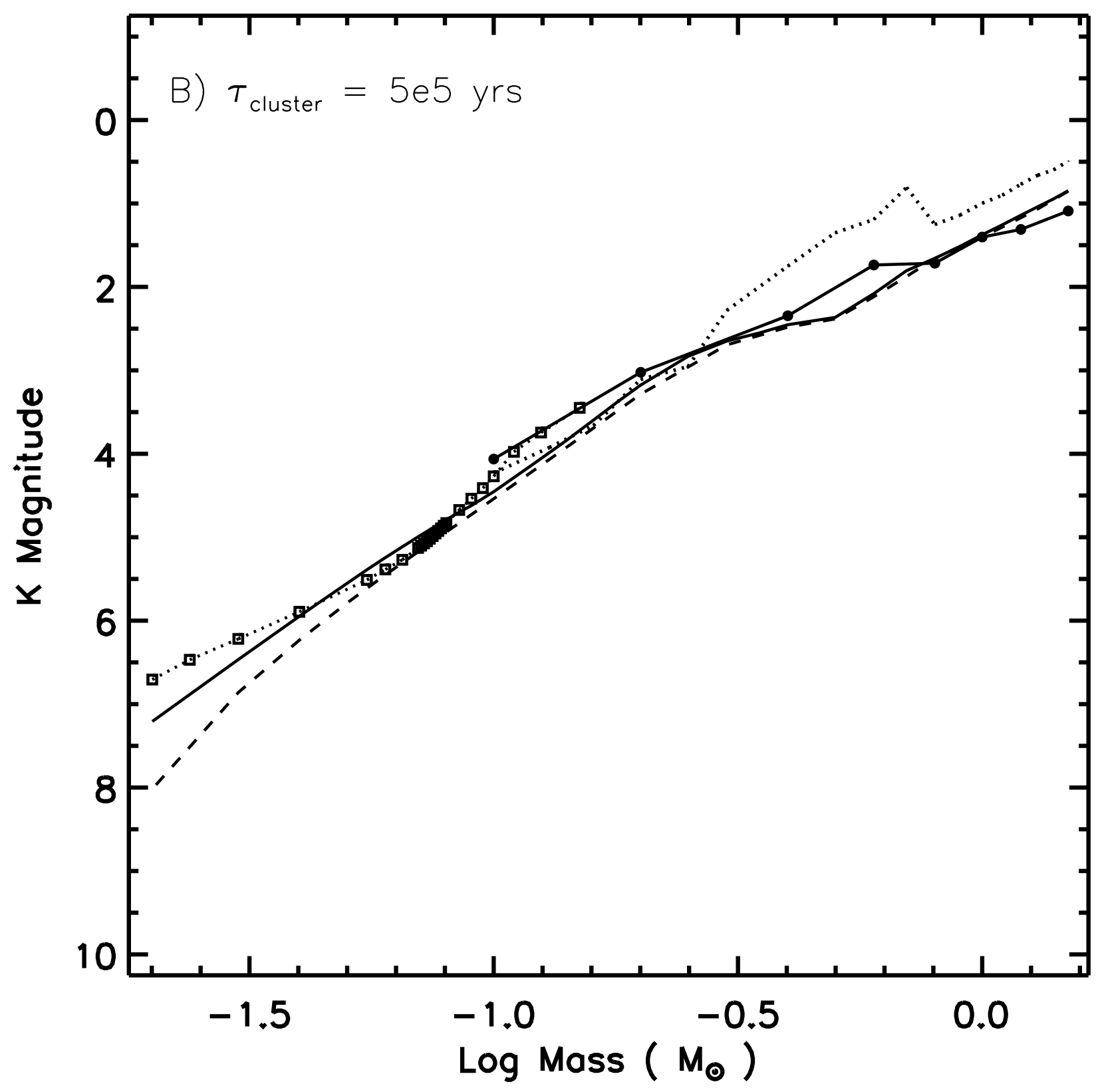




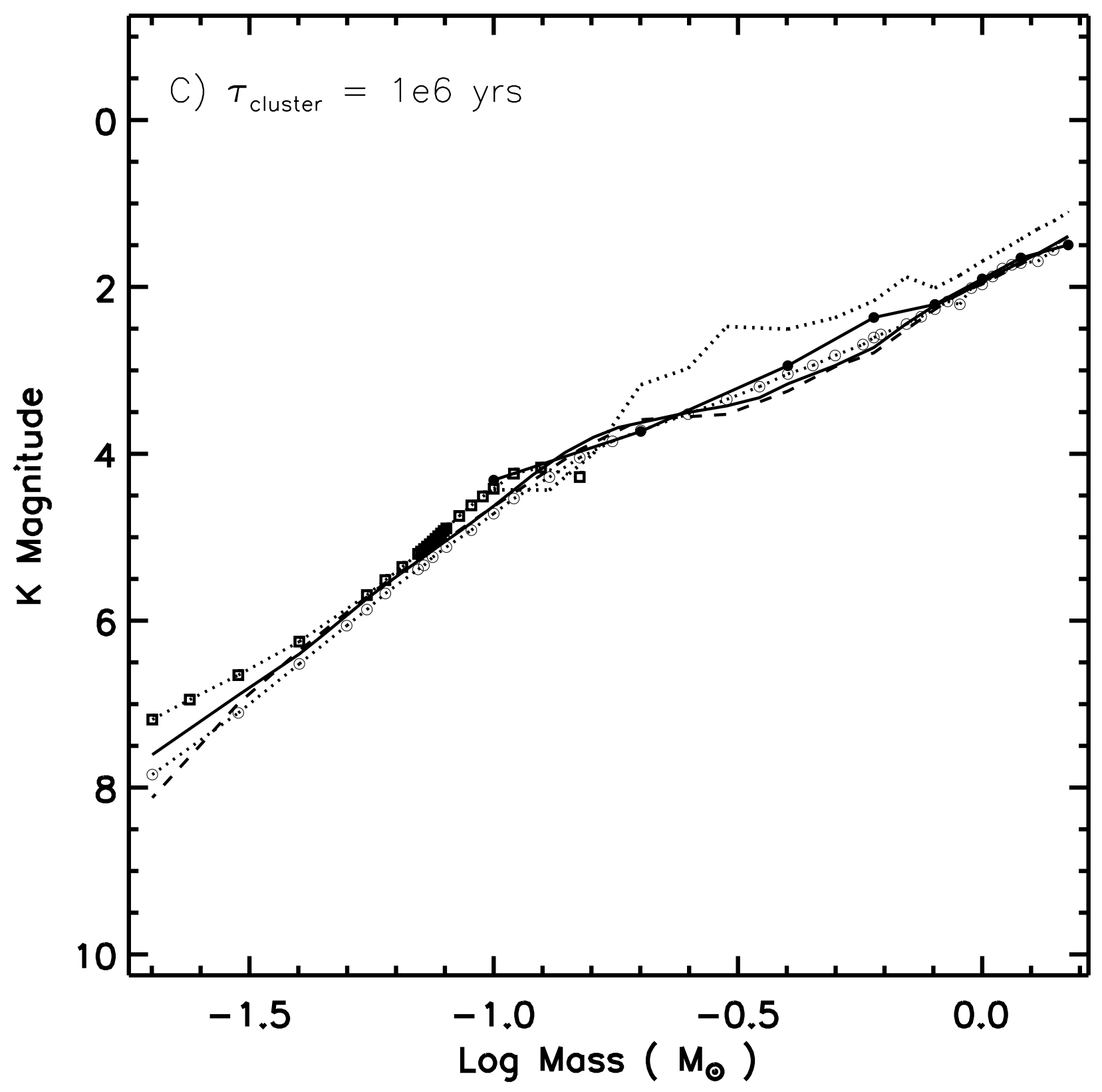




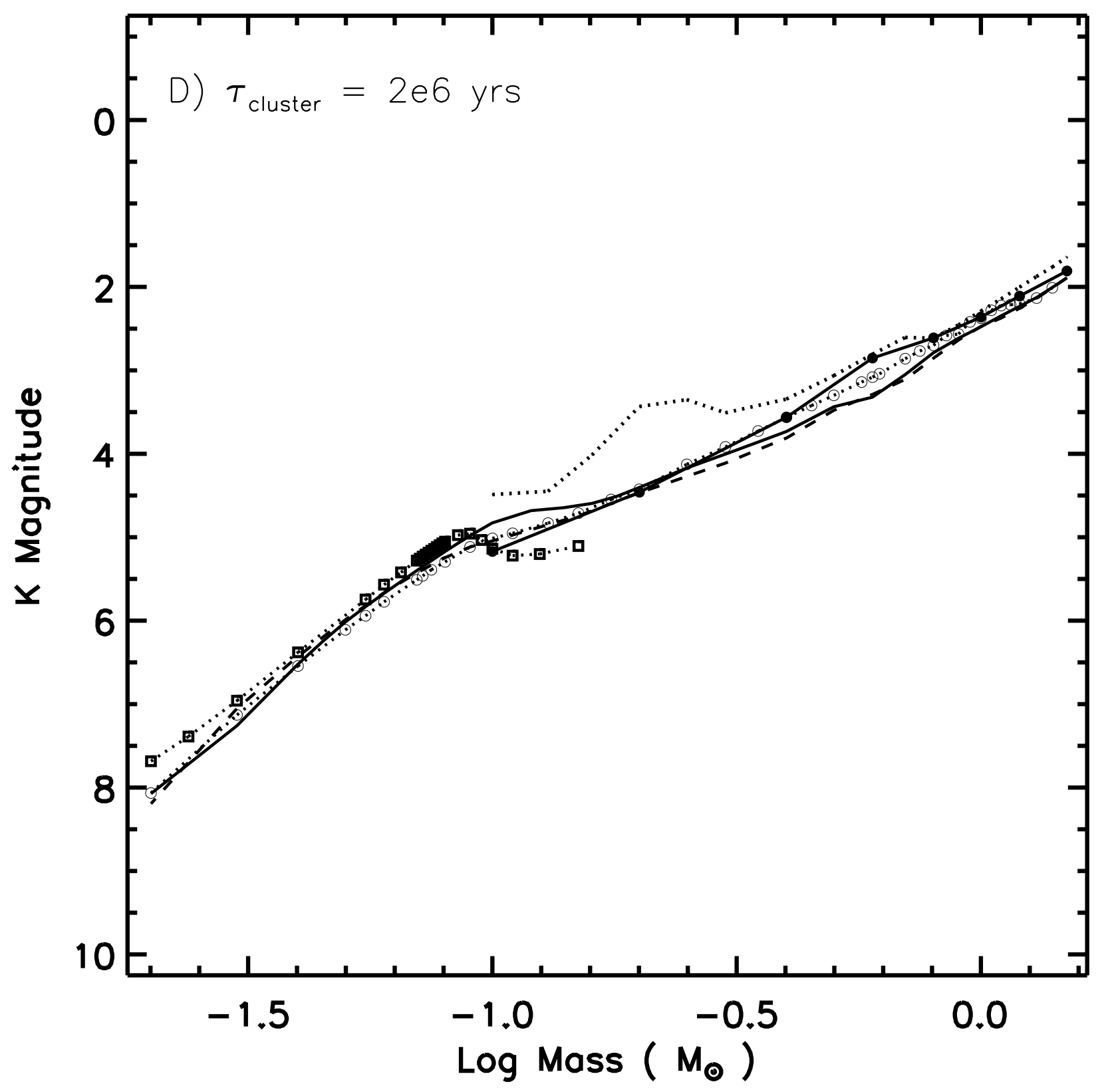




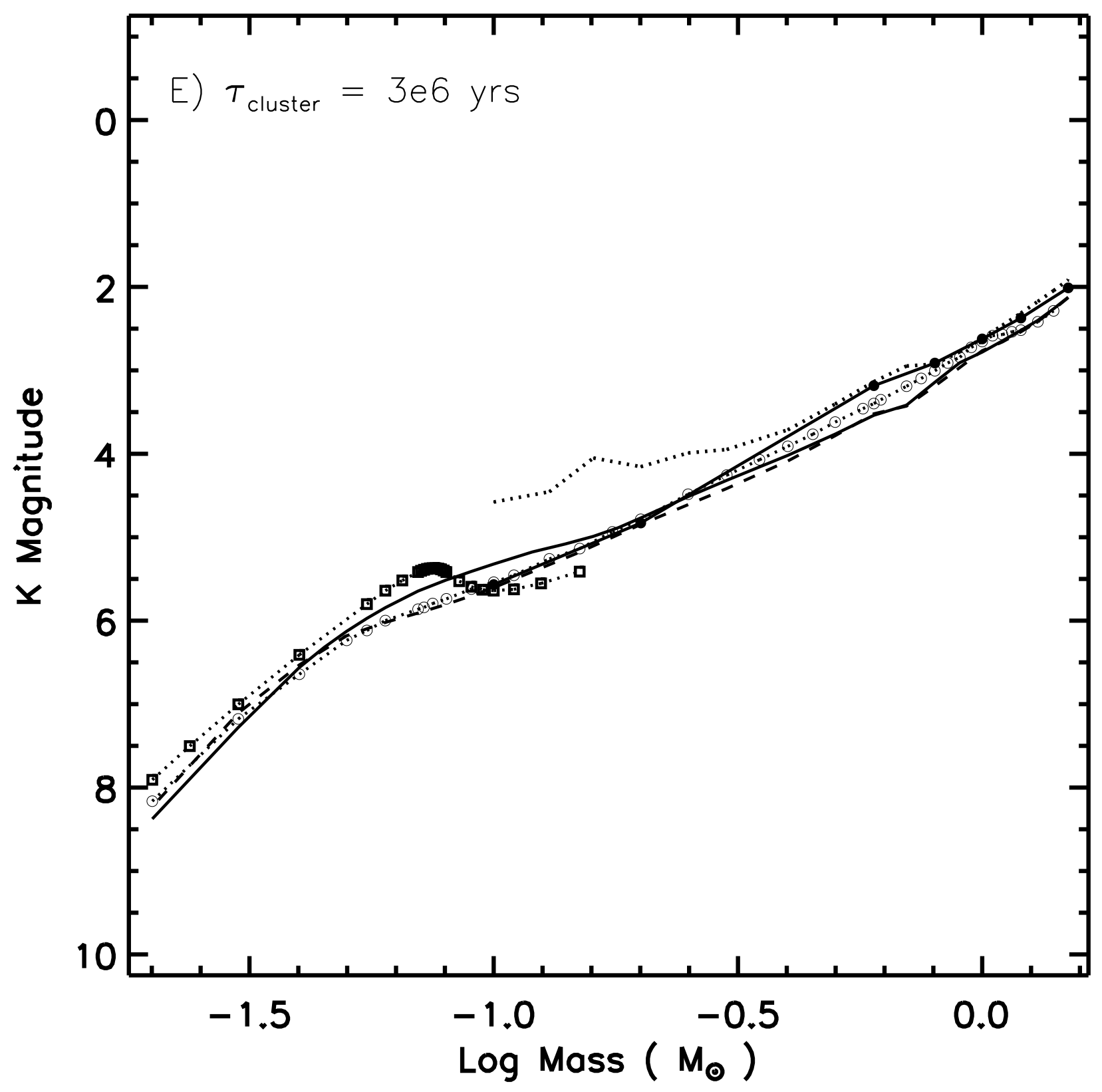




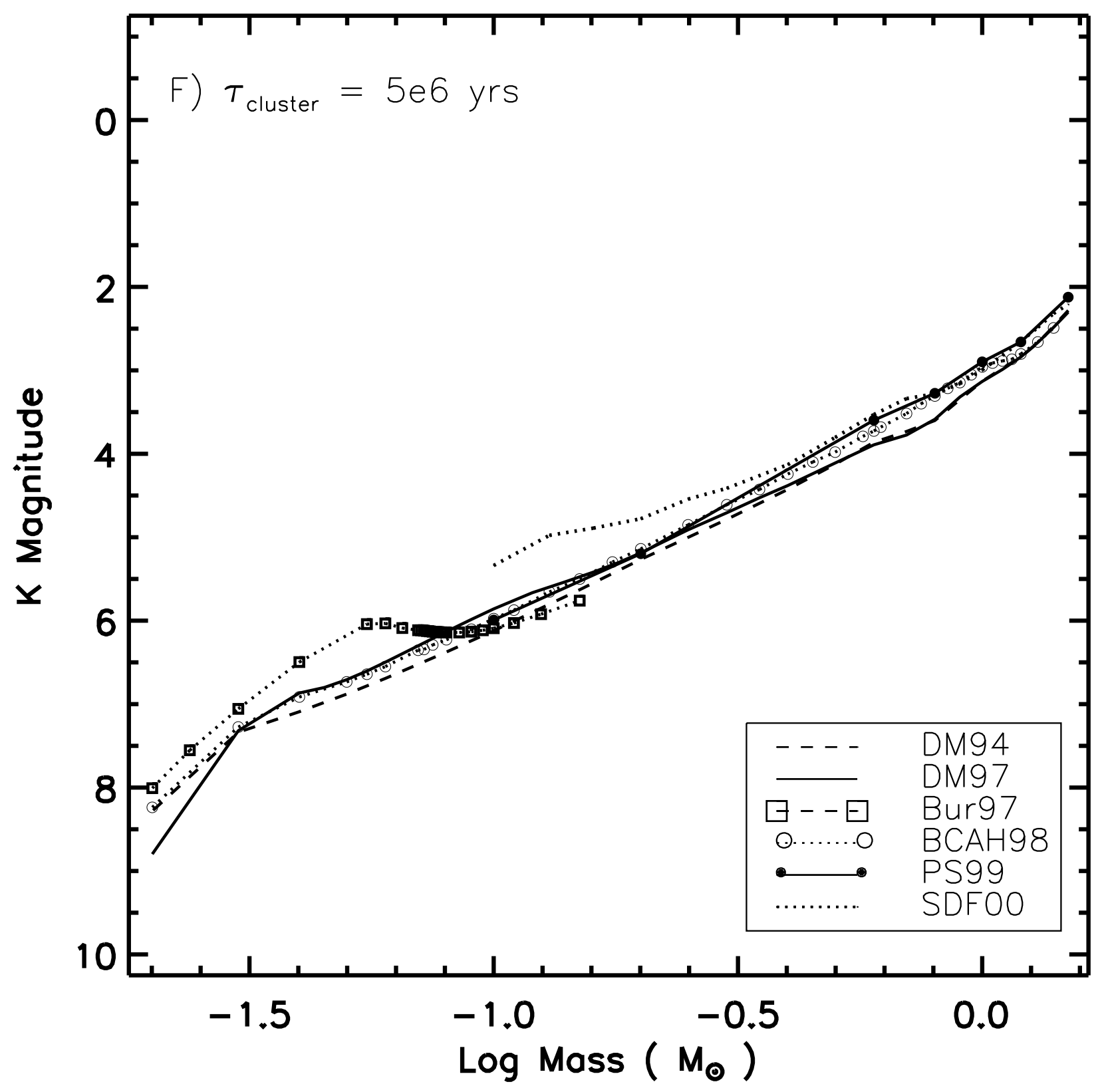




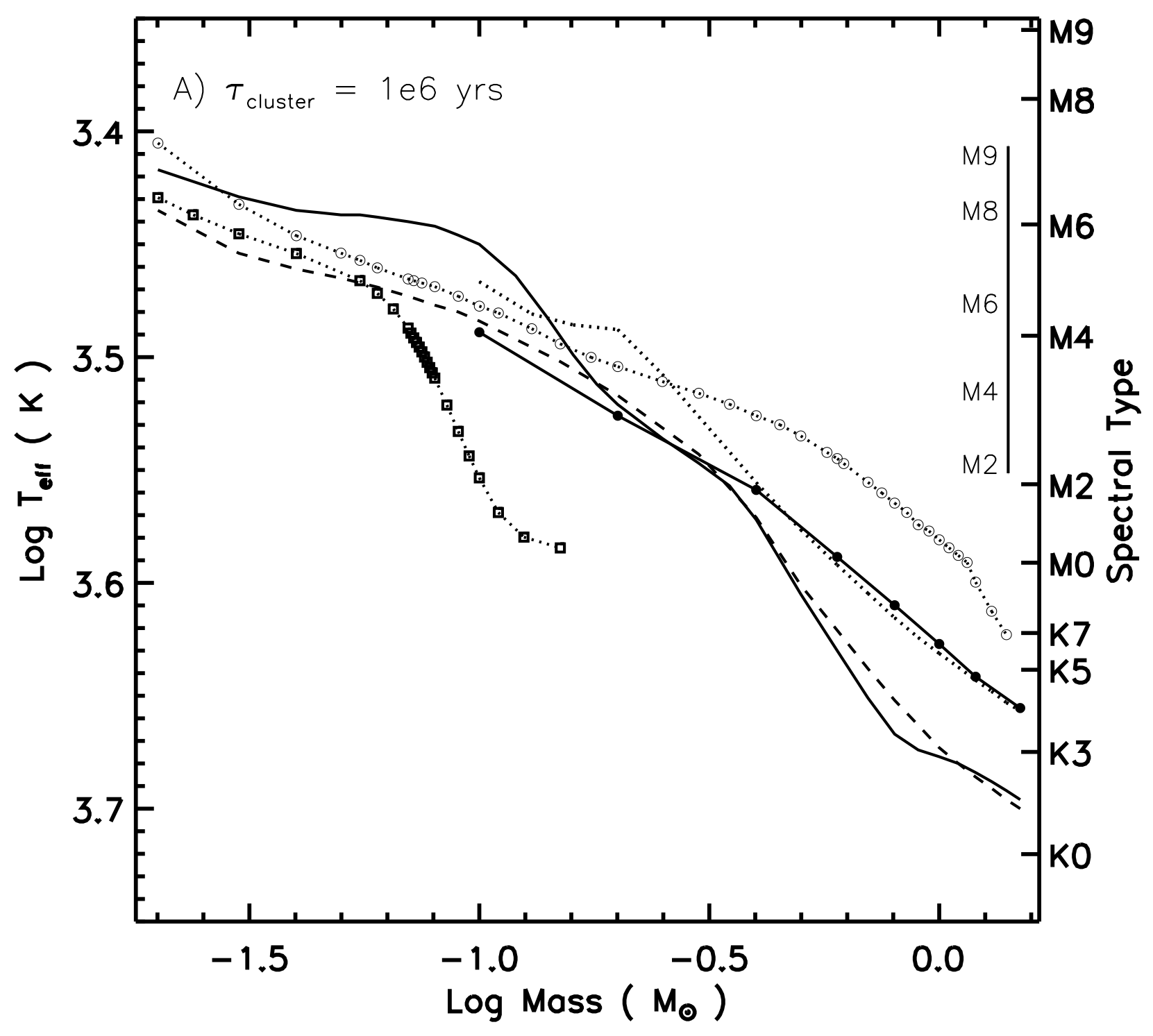




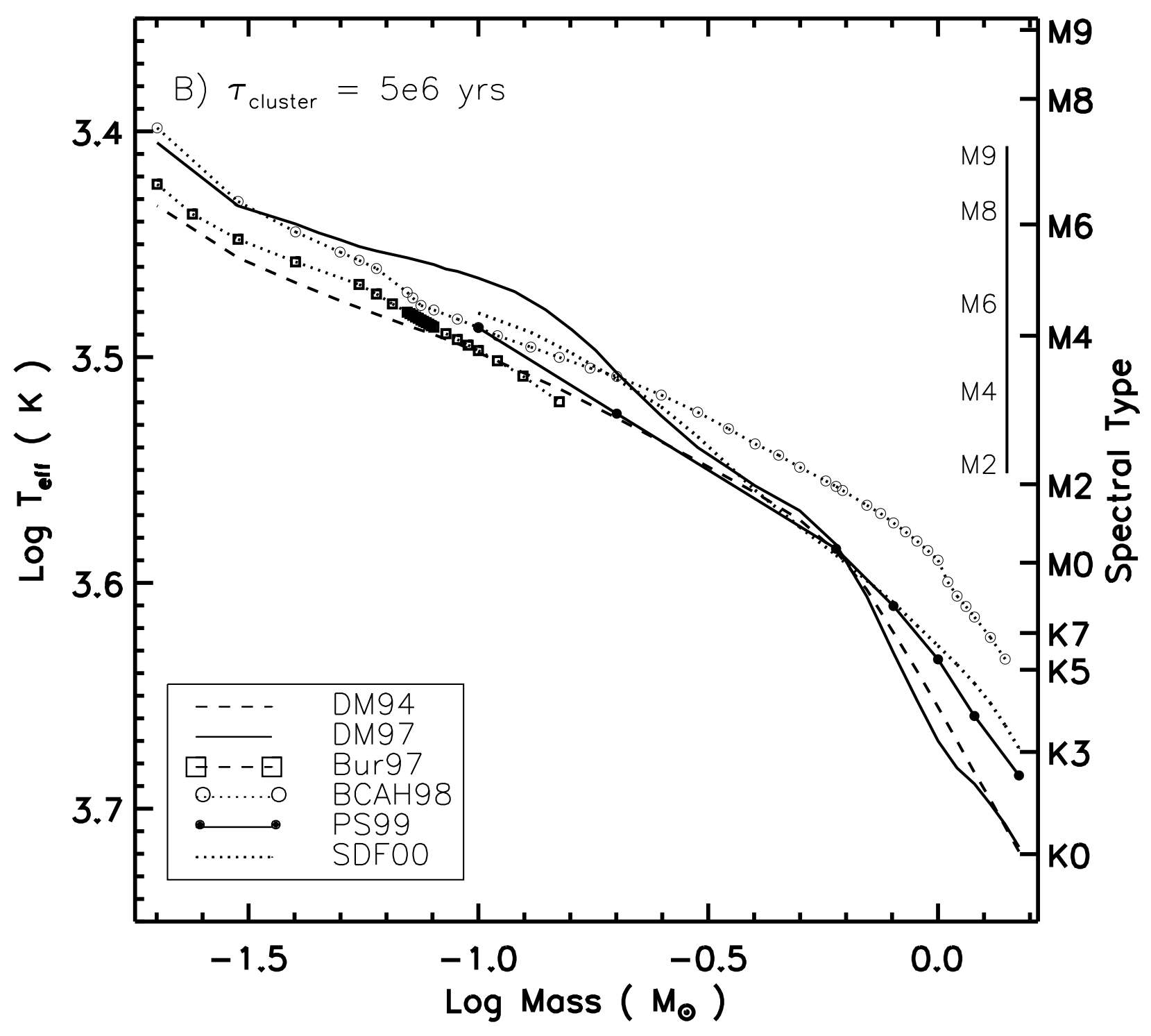




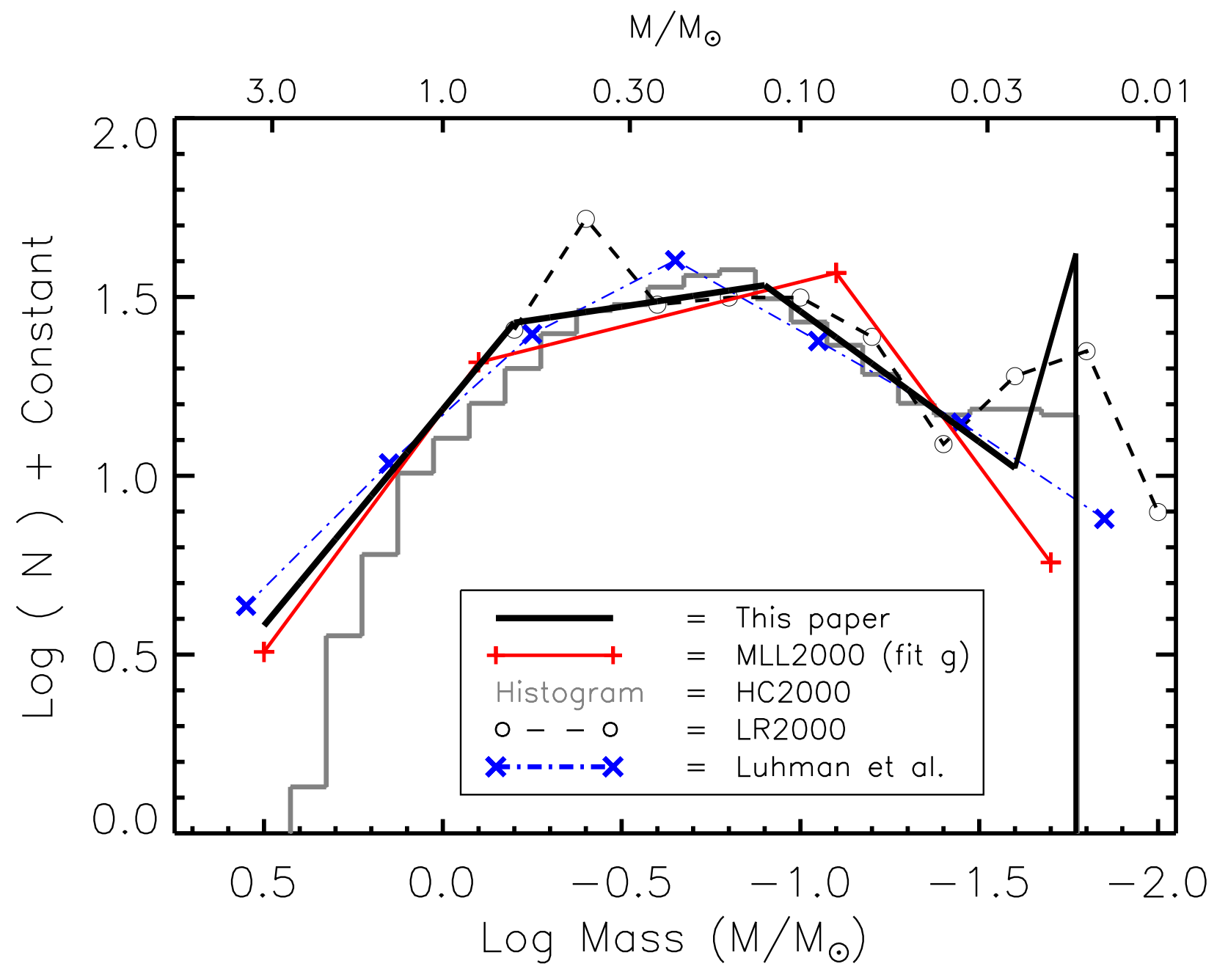




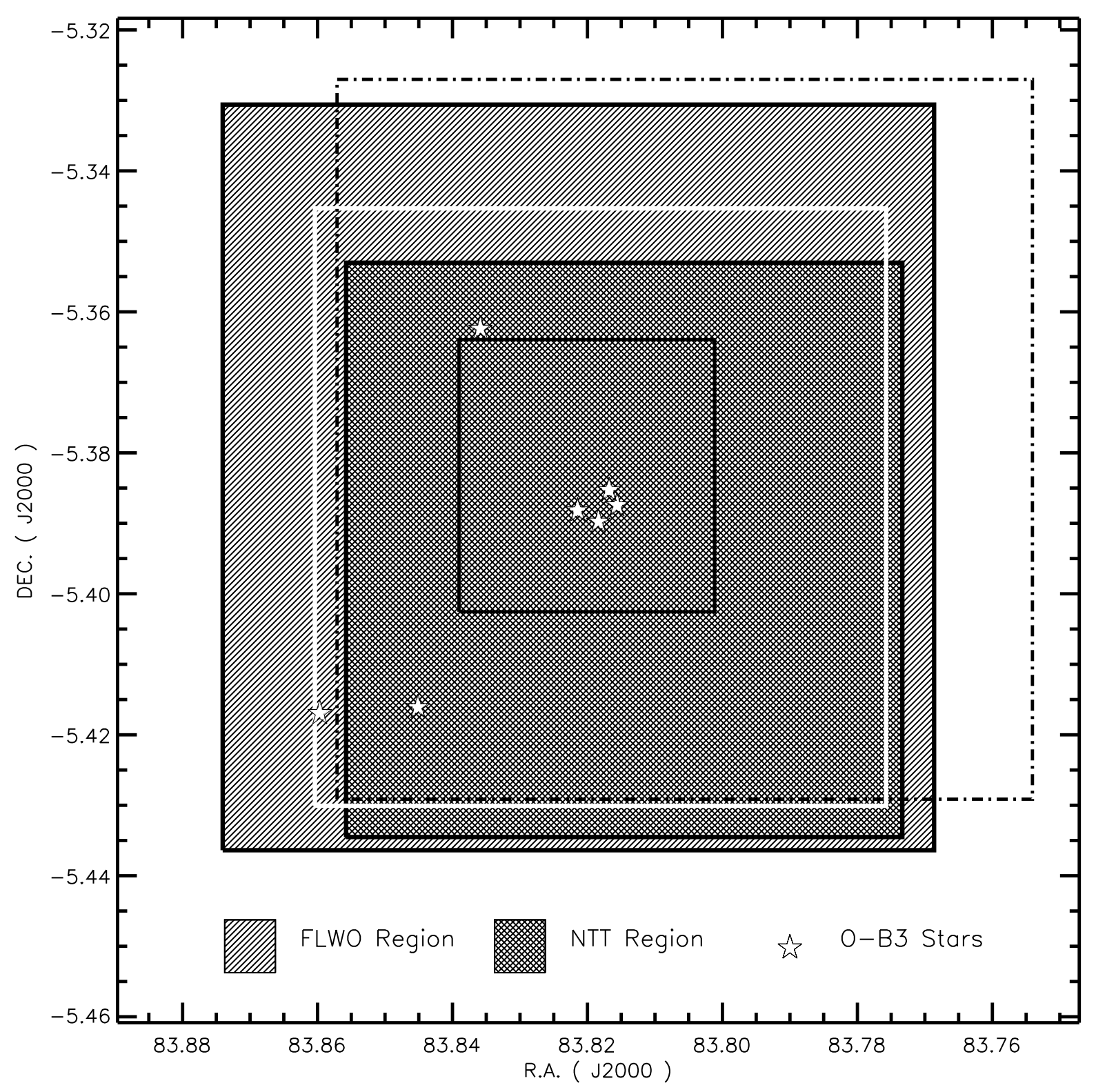


This figure "figure01.jpg" is available in "jpg" format from: http://arxiv.org/ps/astro-ph/0203122v1 